\title{
Self-Assembly of Functional Discrete Three-Dimensional Architectures in Water
}

DOI:

10.1002/anie.201806297

\section{Document Version}

Accepted author manuscript

Link to publication record in Manchester Research Explorer

\section{Citation for published version (APA):}

Taylor, L. L. K., Riddell, I. A., \& Smulders, M. M. J. (2018). Self-Assembly of Functional Discrete ThreeDimensional Architectures in Water. Angewandte Chemie - International Edition.

https://doi.org/10.1002/anie.201806297

\section{Published in:}

Angewandte Chemie - International Edition

\section{Citing this paper}

Please note that where the full-text provided on Manchester Research Explorer is the Author Accepted Manuscript or Proof version this may differ from the final Published version. If citing, it is advised that you check and use the publisher's definitive version.

\section{General rights}

Copyright and moral rights for the publications made accessible in the Research Explorer are retained by the authors and/or other copyright owners and it is a condition of accessing publications that users recognise and abide by the legal requirements associated with these rights.

\section{Takedown policy}

If you believe that this document breaches copyright please refer to the University of Manchester's Takedown Procedures [http://man.ac.uk/04Y6Bo] or contact uml.scholarlycommunications@manchester.ac.uk providing relevant details, so we can investigate your claim.

\section{OPEN ACCESS}




\section{Application Driven Self-Assembly of Discrete, Three-Dimensional}

\section{Architectures in Water}

Lauren L. K. Taylor, ${ }^{[a]}$ Imogen A. Riddell, ${ }^{*[a]}$ and Maarten M. J. Smulders ${ }^{*}[b]$

[a] L. L. K. Taylor (ORCID: 0000-0003-0586-3246), I. A. Riddell (ORCID: 0000-0002-68010198)

School of Chemistry, University of Manchester

Oxford Road, M13 9PL (United Kingdom)

E-mail: imogen.riddell@manchester.ac.uk

Homepage: https://imogenriddell.wixsite.com/riddellgroup

[b] M. M. J. Smulders (ORCID: 0000-0002-6855-0426)

Laboratory of Organic Chemistry, Wageningen University

P.O. Box 8026,6700 EG Wageningen (The Netherlands)

E-mail: maarten.smulders@wur.nl

Homepage: www.smulderslab.nl

Key words: Self-assembly $\cdot$ Water $\cdot$ Cages $\cdot$ DNA architectures $\cdot$ Peptide assemblies 


\section{$\underline{\text { Lead-in text }}$}

Construction of discrete, self-assembled architectures in water has gained significant interest in recent years as a wide range of applications arise from their defined 3D structure. In this review we jointly discuss the efforts of supramolecular chemists and biotechnologists who previously worked independently to tackle discipline-specific challenges associated with construction of assemblies from synthetic and bio-derived components, respectively. Going forward, a more interdisciplinary research approach will expedite development of complexes with real-world applications that exploit the benefits of compartmentalisation. In support of this, we summarise advances made in the development of discrete, water-soluble assemblies, with particular focus on their current and prospective applications. Areas where understanding and methodologies can be transferred from one sector to the adjacent field are highlighted in anticipation this will yield advances not possible from either field alone.

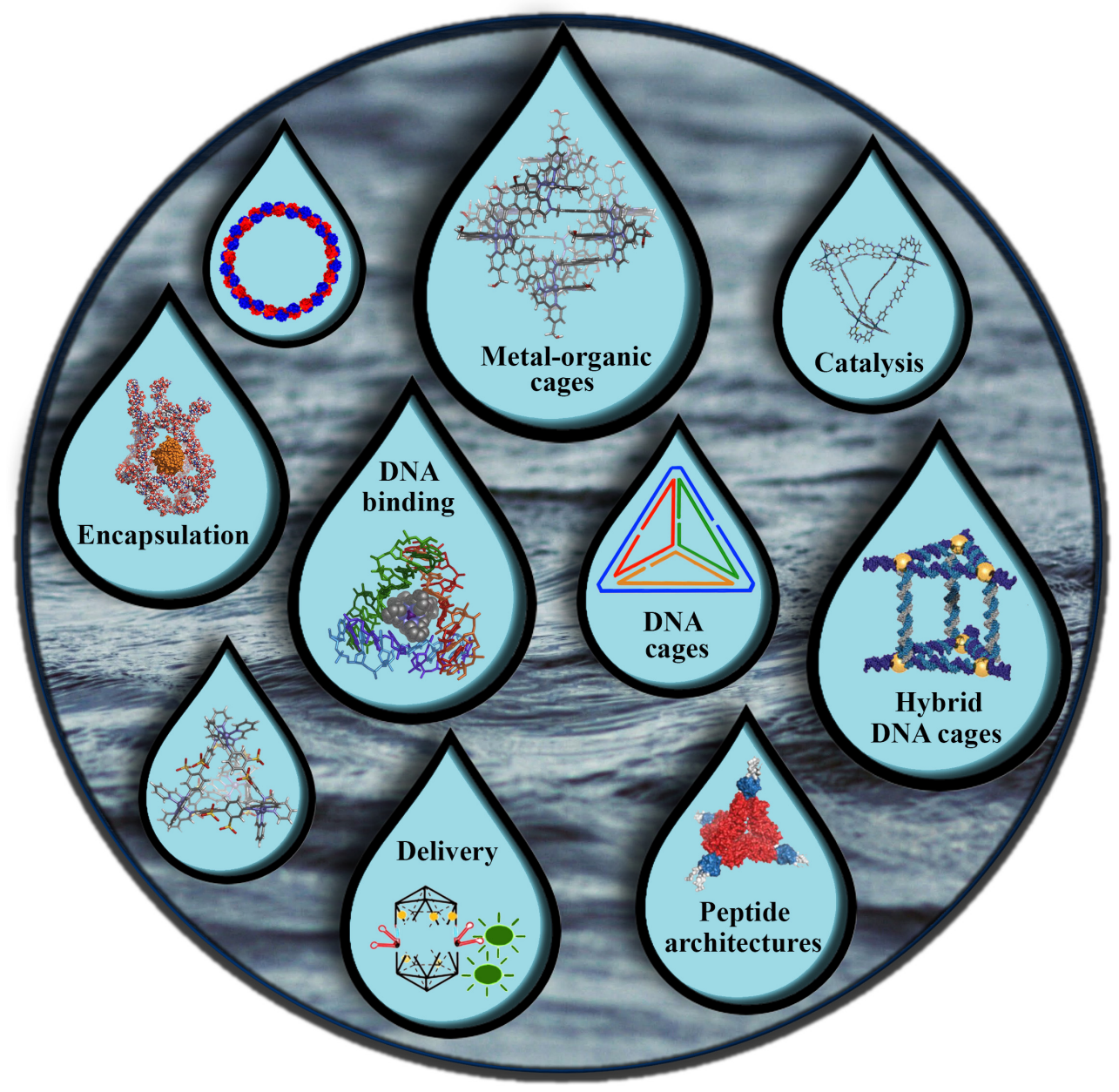




\section{$\underline{\text { 1. Introduction }}$}

Discrete self-assembled three-dimensional architectures, both those comprised of synthetic organic components and those derived from biological building blocks, are key target entities towards high-value applications. These include targeted drug delivery, ${ }^{[1]}$ catalysis ${ }^{[2]}$ and analyte sensing, ${ }^{[3]}$ and the realisation of emergent behaviour such as out-of-equilibrium selfassembly ${ }^{[4]}$ and differential cellular uptake. ${ }^{[5]}$ In particular, of all possible solvents, selfassembly in water presents excellent opportunities to develop environmentally friendly, and biologically relevant assemblies that can exploit the unique hydrogen bonding properties of water, or promote guest binding through the hydrophobic effect.

Water is essential for life, with many equatorial countries facing challenges associated with sourcing and distributing clean water, a challenge scientists have also begun to play a role in addressing in recent years. ${ }^{[6]}$ Furthermore, as industry looks to greener processes, there is a strong drive to move away from harsh chemical treatments towards milder and often biotechnologically driven advances that encourage the use of aqueous solvents. ${ }^{[7-10]}$ Water is also the solvent of choice for biological systems, ${ }^{[10]}$ where exquisite control of reactivity has been demonstrated in complex reaction mixtures. ${ }^{[11-12]}$ If we seek to emulate this level of complexity we must acknowledge, understand and eventually exploit the physical properties of water that make it unique from most other solvents. ${ }^{[13]}$

For scientists, the use of water as a solvent provides both opportunities and challenges. Water is able to act as both a hydrogen bond donor and acceptor, and can form intermolecular interactions with neighbouring water molecules in addition to readily dissolving other polar molecules. This, however, also means that water can compete with many types of supramolecular interactions that hold self-assembled structures together, putting constraints on the applicable molecular entities. It furthermore means that the ionic strength and $\mathrm{pH}$ of the medium can vary significantly, just by making small changes in the solute composition. 
The hydrophobic effect, ${ }^{[13-14]}$ which is operative exclusively in aqueous media, may be used to dictate supramolecular interactions between hydrophobic surfaces, whereas competing hydrogen-bonding interactions with the solvent may prevent the desired interaction of more polar species. Significant insights into each of these processes have been gained in recent years through the concerted efforts of the physical chemistry community. As a result, the fundamental properties of water with respect to its behaviour at interfaces, ${ }^{[15]}$ with ions ${ }^{[16-17]}$ and importantly with the biological substrates, with which it so fruitfully interacts ${ }^{[12,18-19]}$ are now well characterised. Recently, the underexploited synergy between the physical chemical and the supramolecular communities in understanding these intricacies of aqueous selfassembly, was highlighted. ${ }^{[13]}$
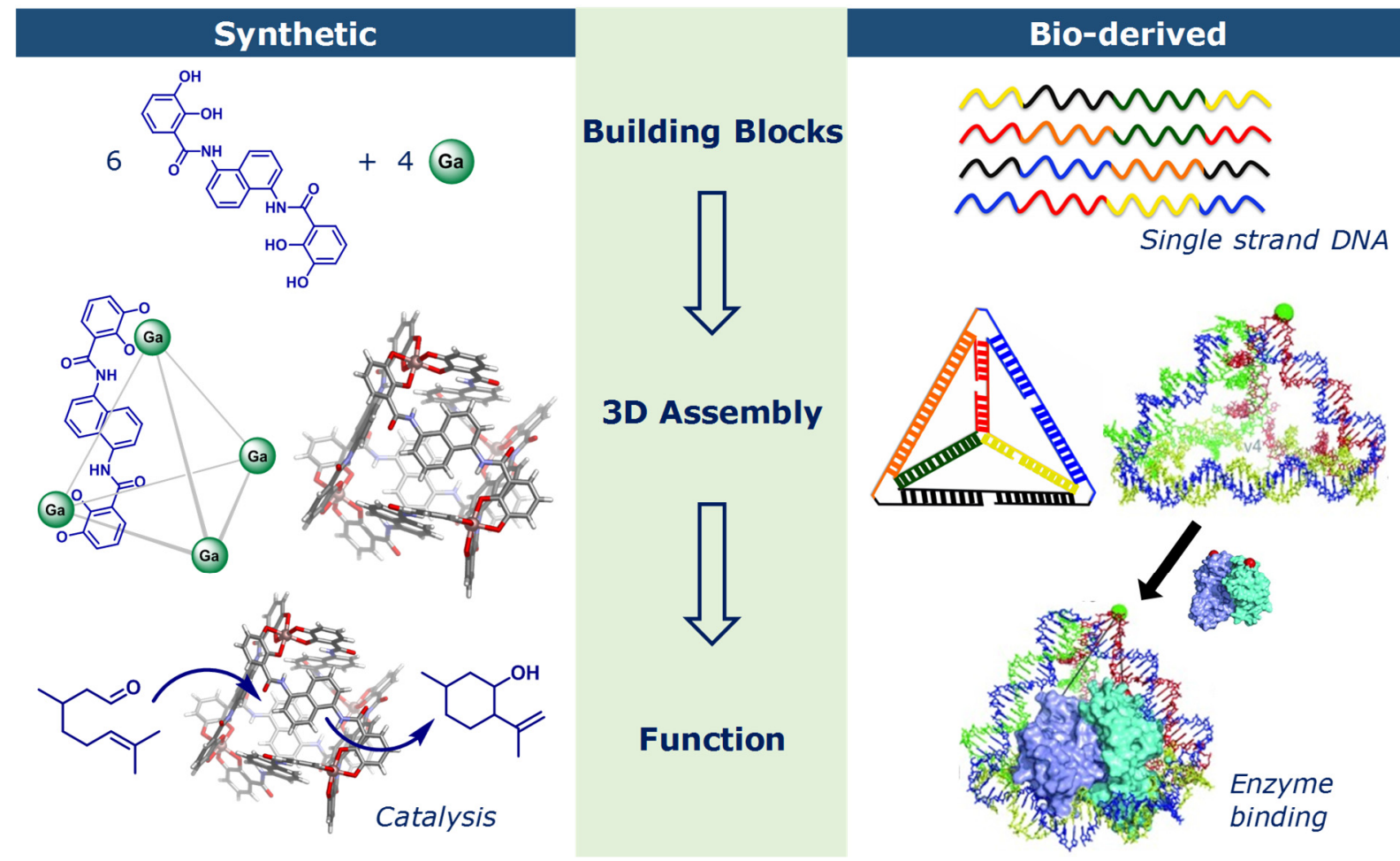

Figure 1. Overview summarising the assembly of synthetic (left) and natural (right) building blocks to generate water-soluble structures containing defined cavities capable of imparting specific function.

Historically, a distinction has been made between discrete assemblies that were derived from natural building blocks (e.g. DNA and protein assemblies $\left.{ }^{[20-22]}\right)$, and those that employed 
synthesised organic ligands (in combination with metal ions) to generate (metal-)organic helicates and cages (Figure 1). ${ }^{[23]}$ Thus, despite sharing common symmetry-based design approaches, supramolecular chemists ${ }^{[24]}$ and biotechnologists ${ }^{[25]}$ have, to date, independently developed synthetic methodologies that enable the construction of a range of discrete, welldefined, 3D assemblies. Looking beyond their assembly, it is apparent that architectures derived from both synthetic and biological components have three-dimensional structures that are critical for their function. ${ }^{[26-27]}$ In each case, the dimensions of the architecture are essential, with the desired property of the assembly arising from either the generation of a well-defined internal guest binding pocket, or through size and shape complementary pairing of the multicomponent architecture with an externally binding guest molecule. In particular, compartmentalisation has been presented as a rational approach to develop applications ranging from drug delivery, to sensing or catalysis.

While sharing many similarities, there are also critical differences between architectures derived from natural and synthetic components, namely the dimensions and hydrophobicity of the cavity or exterior of the architecture. Much larger architectures are generally reported for biologically derived constructs, typically in the nanometer size regime, ${ }^{[28]}$ versus the Ångström-sized architectures reported commonly for synthetic architectures. ${ }^{[29-30]}$ These differences diversify the range of guest molecules that can be accommodated within the host architectures, thus further expanding the potential applications (Table 1). 
Table 1. Comparison of the main features of discrete, synthetic and bio-derived assemblies.

\begin{tabular}{|c|c|c|}
\hline & Synthetic & Bio-derived \\
\hline Building blocks & Polyaromatic linkers (and metal ions) & DNA / amino acids \\
\hline $\begin{array}{c}\text { Synthesis } \\
\text { challenges }\end{array}$ & Aqueous solubility & Angles/ vertexes \\
\hline $\begin{array}{c}\text { Typical length } \\
\text { dimensions }\end{array}$ & $1-10 \mathrm{~nm}$ & $10-100 \mathrm{~nm}$ \\
\hline $\begin{array}{c}\text { Cavity } \\
\text { environment }\end{array}$ & Hydrophobic & Polar interior \\
\hline
\end{tabular}

In this review, challenges associated with the construction of stable, water-soluble, three-dimensional architectures assembled from a fixed number of synthetic components (section 2), and from bio-derived components based on nucleic and amino acid sequences (section 3) are presented alongside the potential application of these architectures. For each type of assembly, the examples presented are sorted by the application that is derived from their $3 \mathrm{D}$ structure.

We focus on discrete, multicomponent architectures that exhibit unusual properties as a result of their $3 \mathrm{D}$ arrangements, and highlight the recent advances that support development of these constructs for applications including drug delivery, bioimaging and catalysis.

Discussion of supramolecular polymers, ${ }^{[31]}$ and hydrogels ${ }^{[32]}$ as well as binary host-guest recognition ${ }^{[33-34]}$ in water fall outside the scope of this review and readers are directed instead to excellent recent reviews on these topics. ${ }^{[31-34]}$ We have also chosen to exclude naturally occurring protein capsules, which have been expertly reviewed by Steinmetz and coworkers. ${ }^{[35]}$

The key aims of this review are thus two-fold. Firstly, we aim to evaluate and concisely summarise the major advances that have been made in the development of discrete, three-dimensional, water-soluble architectures, placing particular emphasis on their current 
and prospective applications. Secondly, this review article aims to emphasise mutual areas of research shared amongst the physical, biotechnological and supramolecular communities, with the expectation that advances made in one sector may bring added value in the adjacent research areas.

\section{Synthetic assemblies}

Current understanding in molecular and supramolecular synthesis has developed to the extent that a broad selection of synthetic species -ranging from mechanically interlocked molecular $\operatorname{architectures}^{[36]}$ to supramolecular polymers, ${ }^{[37]}$ and from cage-like assemblies ${ }^{[23]}$ to

foldamers ${ }^{[38]}$ can now be readily prepared under controlled conditions and characterised with atomic precision. Bringing these assemblies to water remains a challenge, as water can compete with the non-covalent interactions employed in the assembly process. Additionally, the rigid/preorganised ligands commonly employed, that enable the rational design of these architectures, often have poor water-solubility.

Considering the scope of this review, it is evident that because of the necessity of both ligands and metal ions in order to obtain a discrete self-assembled complex, metal-organic assemblies are by their very nature discrete, multicomponent assemblies, and consequently are treated in this section. In contrast, we do not discuss (metal-free) molecular containers that form 1:1 or 2:1 complexes with a guest. These containers, which also assemble in aqueous media through the hydrophobic effect, have been reviewed previously by Jordan and Gibb. ${ }^{[34]}$

The first examples of metal-organic assemblies in water were discussed in the 2007 review on Supramolecular Chemistry in Water, by Oshovsky, Reinhoudt and Verboom. ${ }^{[39]}$ We have therefore chosen to focus on the major developments that these cage complexes have undergone in the subsequent 10 years. In contrast to the 2007 review, which to a large degree focussed on the synthesis of water-soluble cages, we focus our discussion on the emerging applications of water-soluble assemblies that have been reported in recent years. In this 
context, our discussion on discrete, water-soluble cages reflects the development of the field of metal-organic cages, which has now matured beyond assembly and characterisation and instead has a strong focus on the specific application of these cages. ${ }^{[2,40-43]}$ As will be clear from the examples presented below, the advancement in the area of discrete, water-soluble metal-organic complexes is to a large extent related to the various opportunities offered by the hollow interior that is defined for these 3D assemblies. In contrast to analogous 3D assemblies derived from DNA or peptides (see section 3), synthetic cages commonly have a hydrophobic binding pocket that strongly binds apolar, hydrophobic guests in water.

\subsection{Design of water-soluble three-dimensional cages}

Since the ground-breaking work by Saalfrank, ${ }^{[44]}$ Raymond ${ }^{[45]}$ and others in the early nineties, the field of $3 \mathrm{D}$ coordination complexes has rapidly expanded. ${ }^{[23,40,46]}$ These cage-like assemblies can be synthesised when metal ions and polytopic, organic ligands are assembled in the appropriate stoichiometry through formation of a number of coordination bonds. The result is often a highly symmetrical 3D object, such as the archetypical tetrahedral cage (Figure 2). Rational design of edge-bridged tetrahedral cages requires six ligands with appropriate coordination moieties to bridge between four metal ions that occupy the vertices of the tetrahedron. ${ }^{[45]}$ Explicitly, a tetrahedral assembly utilises octahedral metal ions typically in combination with bidentate coordination motifs allowing three such bidentate motifs to be assembled around each metal centre. Linking two of these bidentate moieties together with a rigid linker, to create bisbidentate ligands, enables the ligands to link two metal ions together. As a result, from the metal ion's perspective three coordination motifs wrap around a single metal, while at same time a single ligand can bridge two metal ions; exactly as is required for a tetrahedral cage (Figure 2). Varying the polytopic ligands, metal ion coordination geometry 
and metal-to-ligand ratio has enabled a wide variety of polynuclear cage-like assemblies to be prepared. ${ }^{[23]}$

As a subset of the numerous reported self-assembled metal-organic cages, the number of water-soluble cages is relatively limited. To some extent, this can be understood by considering the rigid ligands that are frequently used to link the metal ions together. Rigidity is generally a prerequisite to ensure that the desired complex is formed, but also means that in practice often aromatic ligands, with intrinsically limited water solubility, are used. This poor water solubility can partially be overcome upon complex formation with charged metal ions. However, additional efforts are often needed to ensure sufficient water solubility, e.g. equipping the aromatic ligands with solubilising side groups, ${ }^{[47-48]}$ including charged (hetero)atoms in the aromatic ring, ${ }^{[49]}$ or exchanging the cage's counterions (vide infra). ${ }^{[30,50]}$

\subsection{Catalysis}

A key feature of most coordination cages is their internal void pocket. The unique properties of these cavities have been exploited for a range of different applications, including guest binding and separation, ${ }^{[51-52]}$ cavity-controlled catalysis, ${ }^{[41,53]}$ generation of unusual reaction products $^{[54-55]}$ and stabilisation of reactive intermediates. ${ }^{[56-57]}$ Inspired by the excellent catalytic control over reactivity in the active site of proteins, the use of hydrophobic binding pockets in coordination cages has been explored for catalysis.

One of the first cages that was reported by Bergman, Raymond and co-workers, to promote catalysis in aqueous medium was a tetrahedral, $\mathrm{M}_{4} \mathrm{~L}_{6}$-type cage $\mathbf{2},{ }^{[45]}$ derived from naphthalene-based catechol ligand 1 that coordinates to $\mathrm{Ga}$ (III) ions (Figure 2A). Building on the observation that this polyanionic cage can stabilise protonated guests, they employed this cage to accelerate reactions that occur via positively charged transition states that could be potentially stabilised by encapsulation. ${ }^{[58]}$ Moreover, the cage's high negative charge induced 
a drastic $\mathrm{p} K_{\mathrm{a}}$ shift for encapsulated guests, ${ }^{[59]}$ consequently, this water-soluble cage is also highly suited to perform acid-catalysed hydrolysis reactions. In addition, the Nazarov reaction, an acid-catalysed cyclisation reaction, was accelerated by a factor of more than $2 \times$ $10^{6}$, placing synthetic capsules on a par with biological, enzymatic systems in terms of catalytic performance. ${ }^{[60]}$

A<smiles>O=C(Nc1ccccc1NC(=O)c1ccccc1O)c1ccccc1O</smiles>

1

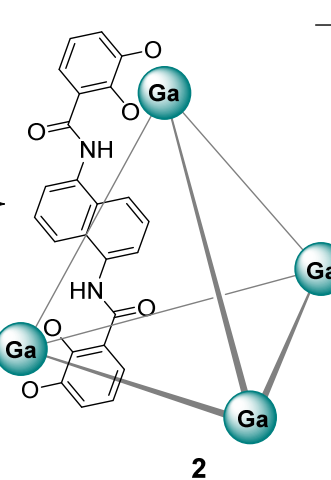

2

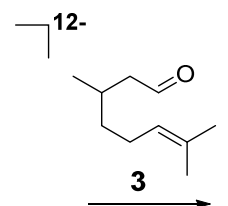

3

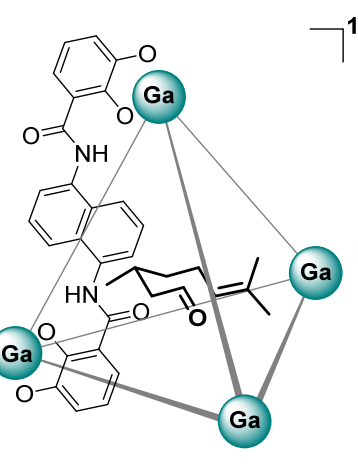

$3 \subset 2$

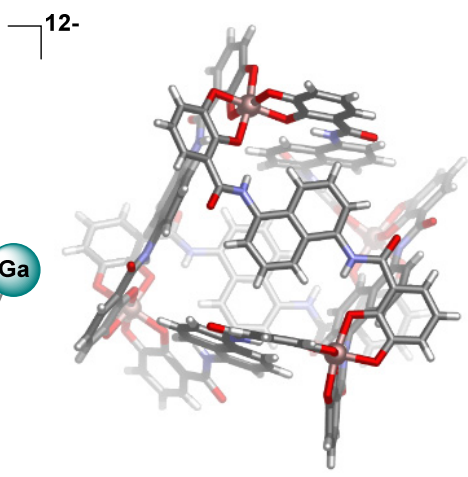

B

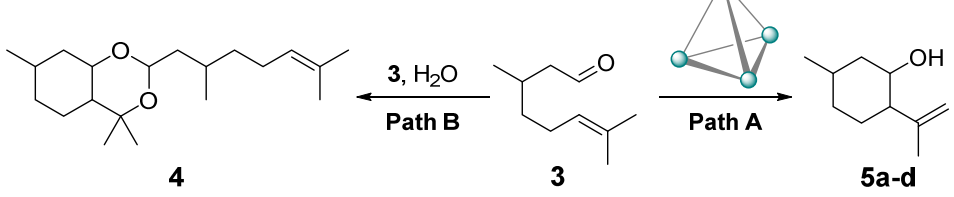

Figure 2. A: Synthesis, schematic representation (left) and single crystal X-ray structure (right) of the polyanionic $\mathrm{Ga}_{4} \mathrm{~L}_{6}$ cage reported by Raymond, and the subsequent encapsulation of the monoterpene $( \pm)$-citronellal (3). ${ }^{[61]}$ The chemical structure of only one of the ligands that bind to two metal ions is shown; the other five are omitted for clarity and are instead represented by a solid line. B: Two possible synthetic pathways for $( \pm)$-citronellal 3: in the presence of cage $\mathbf{2}$ cyclisation is favoured (path A), while in the absence of cage $\mathbf{2}$, nucleophilic attack by $\mathrm{H}_{2} \mathrm{O}$ ultimately leads to the formation of the condensation product 4 . Please note that four stereoisomers of $\mathbf{5}$ will be formed.

Toste, Bergman, Raymond and co-workers have also demonstrated how cage $\mathbf{2}$ can perform like a terpene synthase enzyme. ${ }^{[61]}$ The authors astutely note that the hydrophobic properties and cation-stabilising properties of the active sites of terpene synthases can be modelled by the polyanionic $\mathrm{Ga}(\mathrm{III})$ cage. Indeed, in common with terpene synthases, cage 2 steers the cyclisation of monoterpene 3 towards deprotonation, instead of nucleophilic attack by water (Figure 2B), illustrating how the internal cavity of the cage offers a shielded hydrophobic environment that can divert the course of the reaction with $\mathbf{3}$ in water. 
Analogously, the gold-catalysed cycloisomerisation of an enyne proceeds in the absence of water in the hydrophobic cage interior, generating distinct reaction products. Additionally, through steric confinement conformational control over substrate reactivity was achieved: cage 2 afforded product selectivity in both the presence and the absence of gem-dimethyl substitution. In a similar fashion, Mukherjee and co-workers have demonstrated dehydration reactions in aqueous medium using a water-soluble prismatic cage that catalyses the reaction by excluding water from the hydrophobic cavity. ${ }^{[62]}$

The highly charged nature of metal-organic cages not only provides water solubility, but can also control reactions. In contrast to the polyanionic tetrahedral cage from Raymond presented above, Hunter, Williams, Ward and co-workers have reported the synthesis of a water-soluble, polycationic cubic cage 6 (Figure $3 \mathrm{~A}$ ). ${ }^{[47]}$ While the first generation of this $\mathrm{Co}(\mathrm{II})$ cube was not water-soluble, ${ }^{[63]}$ attaching hydroxymethyl substituents to the pyridyl $\mathrm{C}_{4}$ sites of the ligand generated the water-soluble analogue $6{ }^{[47]}$ The high cationic charge of the cubic cage, in combination with the hydrophobic cavity, enabled efficient catalysis of the Kemp elimination reaction of benzisoxazole with hydroxide (Figure $3 \mathrm{~B}$ ). ${ }^{[64]}$ The hydrophobic cavity was able to strongly bind to the neutral substrate, but was a poor host for the anionic product, which preferred to be in the water phase. Product inhibition during the catalytic cycle could thus be efficiently prevented. Moreover, the cage not only strongly bound to the substrate, but it simultaneously accumulated a high concentration of partially desolvated hydroxide ions due to ionic interactions with the highly positively charged (16+) construct, thus increasing the effective concentration of the hydroxide ions. This combination of two orthogonal interactions: i) guest binding driven by the hydrophobic effect and ii) polar hydroxide binding, that are uniquely operative in water, enabled the catalytic reaction to occur with a rate enhancement of $k_{\text {cat }} / k_{\text {uncat }}$ of $2 \times 10^{5}$. In a very recent contribution, the same 
researchers established how anions that accumulate at the cage surface can displace the hydroxide anions, thus effectively inhibiting catalysis. ${ }^{[65]}$

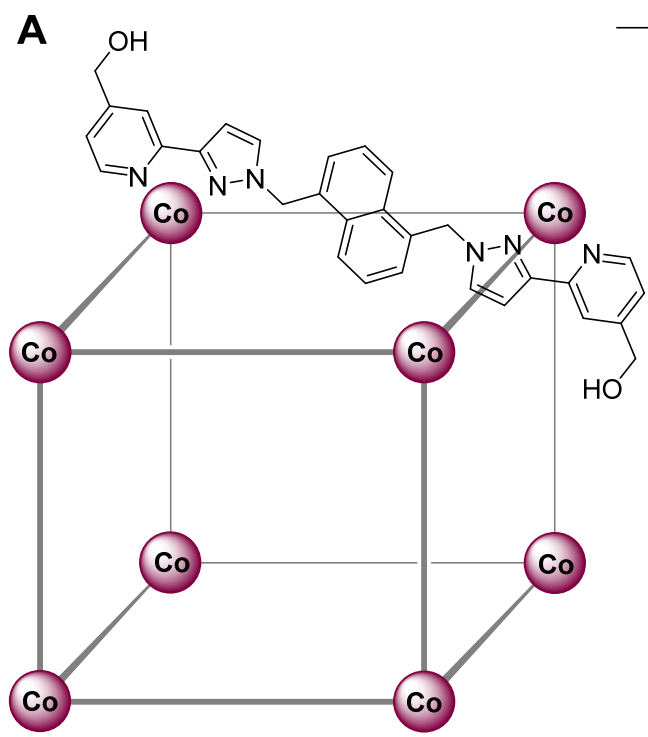

6
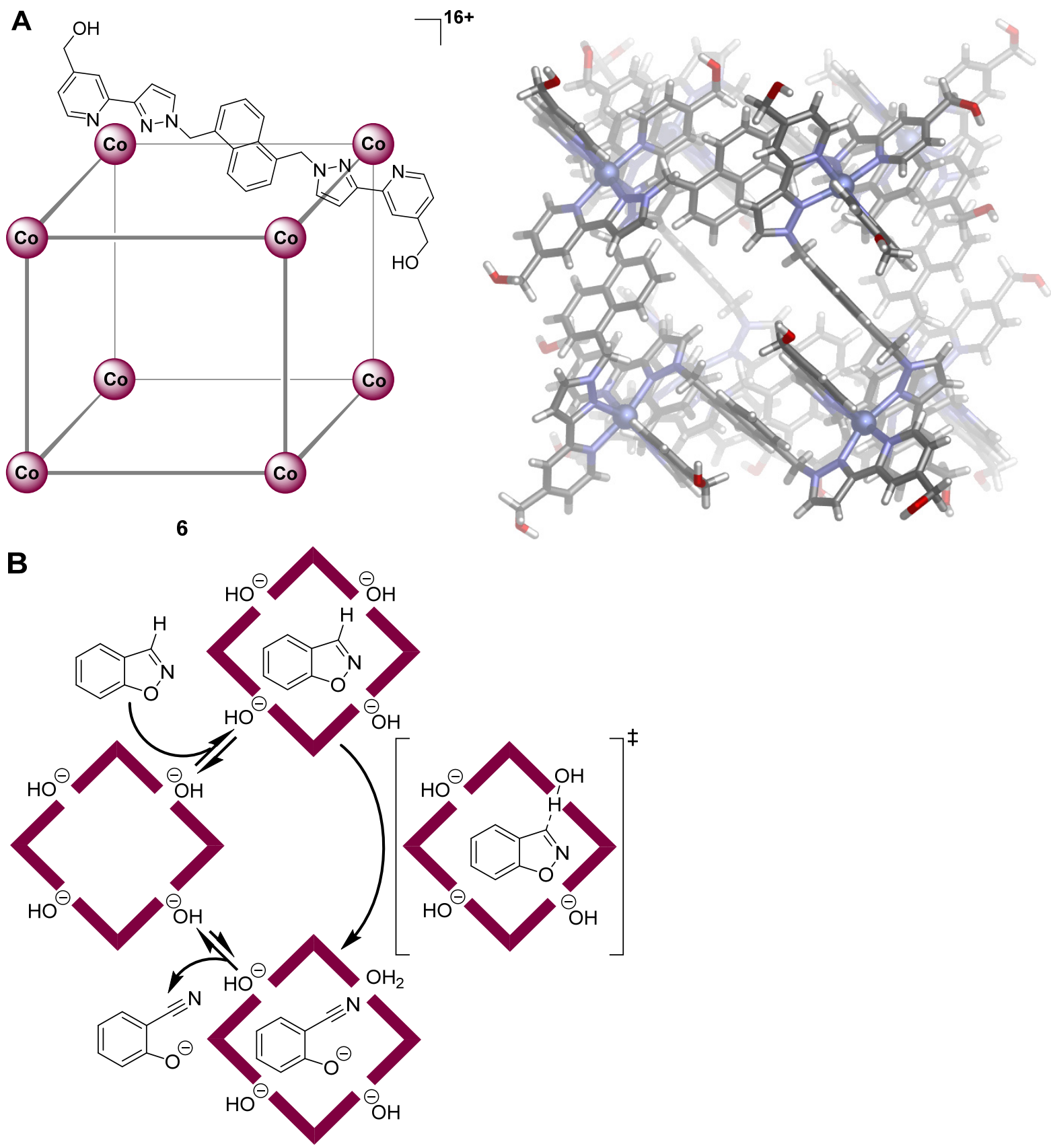

Figure 3. A: Schematic representation (left) and single crystal X-ray structure (right) of the cubic $\mathrm{Co}_{8} \mathrm{~L}_{12}$ cage 6 reported by Ward.$^{[64]} \mathrm{B}$ : Scheme of the reaction cycle that is catalysed inside the cubic cage $\mathbf{6}$. Starting centre left, the hydrophobic benzisoxazole substrate binds in the cavity with the proton at the 3-position exposed to a hydroxide ion that is bound in the adjacent cage window (top). The elimination reaction occurs to produce a 2-cyanophenolate ion as the product (bottom). Due to the negative charge the anion is less hydrophobic and less strongly bound within the cage than the hydrophobic starting material, causing it to become ejected from the cavity. 
In contrast to the homogeneous catalysts reported above, Mukherjee and co-workers have reported a heterogeneous system that allowed straightforward isolation of the products and recovery of the catalyst from water by simple filtration. ${ }^{[66]}$ Their prismatic $\mathrm{Pd}_{6}\left(\mathrm{~L}_{1}\right)_{6}\left(\mathrm{~L}_{2}\right)_{6}$ cage 7 (Figure 4A) featured carefully positioned urea groups that act as hydrogen-bond donors, serving as catalysts for the Michael reaction of a range of water-insoluble nitroolefins (Figure 4B). This pre-organisation of the urea moieties prevents self-quenching of the urea groups through intermolecular hydrogen bond formation, as determined by comparing the catalytic activity of the cage to the corresponding ligand and triangle. The same cage was also shown to catalyse the Diels-Alder reaction of 9-hydroxymethylanthracene with $N$ substituted maleimide in the aqueous medium (Figure 4B). In both cases the reactions are accelerated through binding of the water-insoluble guest in the hydrophobic cavity of the prismatic cage.
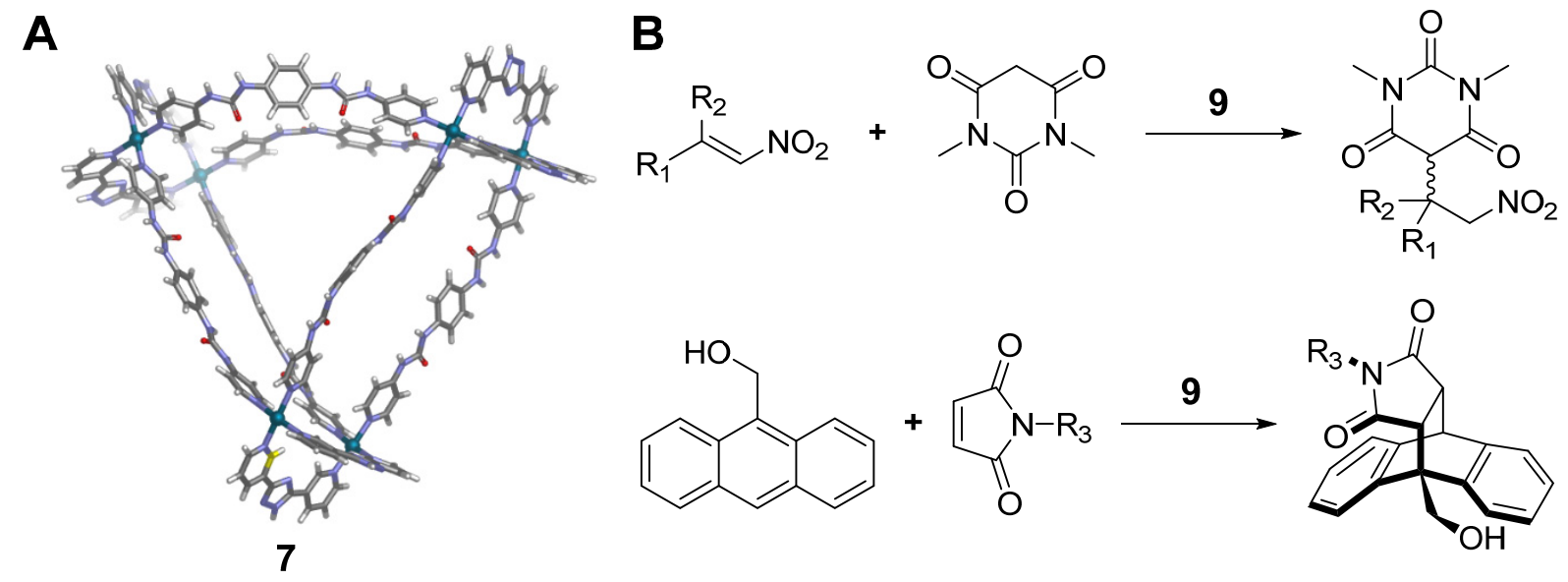

Figure 4. Single crystal X-ray structure (A) of the prismatic cage 9, that can catalyse the Michael addition reaction of nitro-olefins (B, top), as well as the Diels-Alder reaction of 9hydroxymethylanthracene (B, bottom). ${ }^{[66]}$

The same group also reported an alternative approach to prevent product inhibition in cage-catalysed reactions. ${ }^{[67]}$ By designing a tube-like cage structure, trapping of the product inside the cage cavity could be prevented. This enabled efficient control of the regio- as well 
as stereoselective pathways of the intramolecular hetero [4+2] cycloaddition reaction by restricting the conformational flexibility of the diene and linked olefin.

De Bruin and co-workers first reported the "molecular ship-in-a-bottle catalyst" capable of performing size-selective cyclopropanation reactions in aqueous media. ${ }^{[50]}$ In this type of catalytic reaction, the homogeneous catalyst is caged inside a larger coordination complex, thus offering the possibility for shape and/or size selectivity. The faces of the cubic cage system employed comprised of zinc porphyrin rings and a judiciously chosen, catalytically active cobalt tetrapyridine porphyrin that was shown to fit between two pairs of opposing faces inside the cube (cage 10, Figure 5). Moreover, for compatibility in aqueous media (typically water/acetone 5:1), exchange of the counter anion from triflate (OTf ${ }^{-}$) for triflimide $\left(\mathrm{NTf}_{2}{ }^{-}\right)$was performed. The cobalt centre inside the cube was capable of performing size-selective radical-type cyclopropanation reactions on styrene molecules. The yield of the reaction was reported to increase with increasing water content of the solvent, as the water formed stabilising hydrogen bonds with the reaction intermediates. In a follow-up paper, the same group demonstrated that the manganese analogue cage $\mathbf{1 1}$ could successfully act as phase transfer catalyst for catalytic epoxidation reactions in 1:1 water/acetonitrile mixtures. ${ }^{[68]}$ 


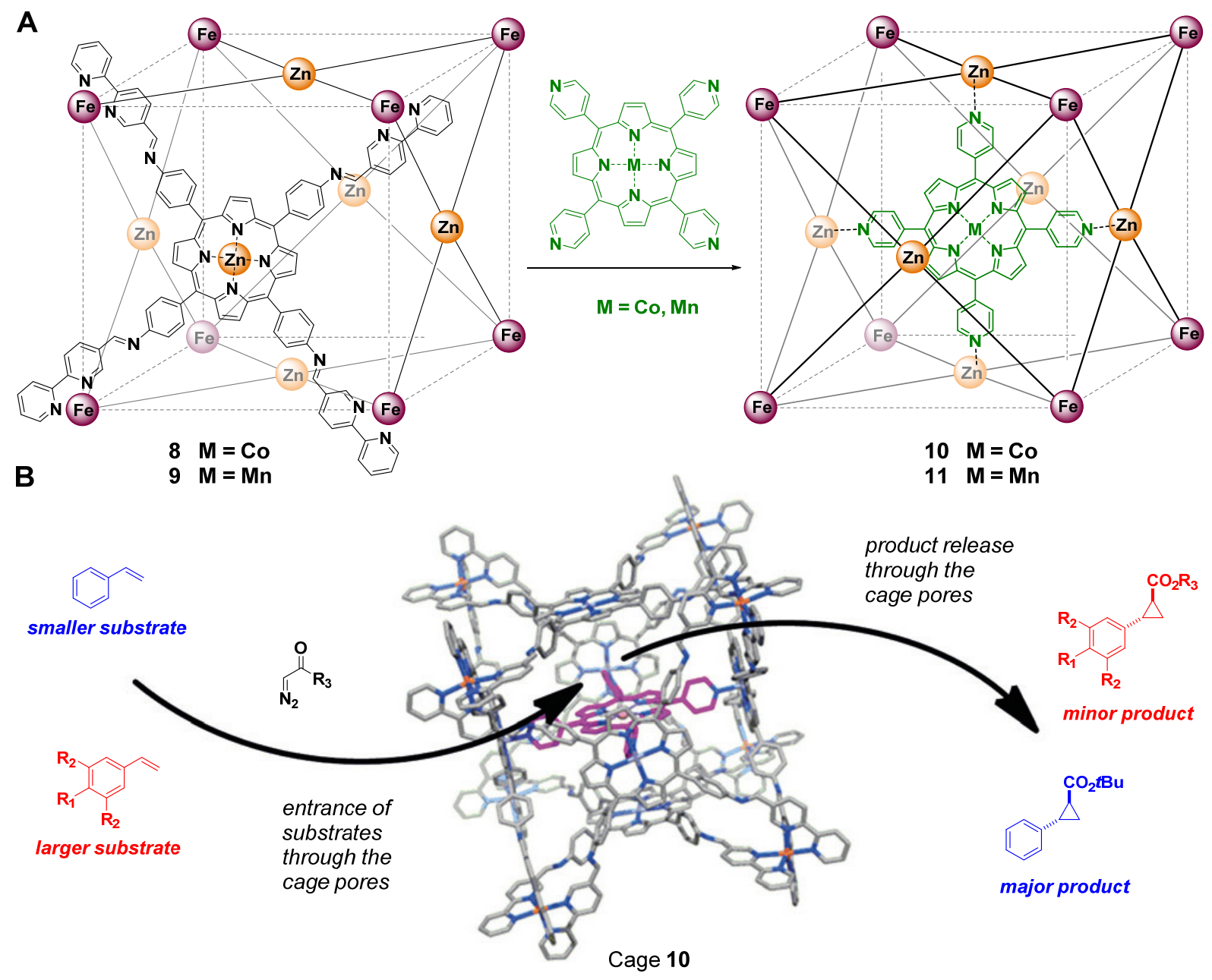

Figure 5. A: Assembly of the "molecular ship-in-a-bottle" cage complexes $\mathbf{1 0}$ and $\mathbf{1 1}$ from the empty $\mathrm{Fe} / \mathrm{Zn}$ cube $\mathbf{8}$ and $\mathbf{9}$, and a metal-tetrapyridine porphyrin derivative. B: schematic representation of the size-selective transformations catalysed by the "molecular ship-in-a-bottle" cage 10. ${ }^{[50]}$

In order to achieve water solubility of a family of terphenylene-based tetrahedral cages, that had previously been reported to assemble in acetonitrile, ${ }^{[69]}$ Nitschke and coworkers installed chiral glyceryl groups on the central phenyl ring of the terphenyl ligand. ${ }^{[70]}$ These glyceryl groups closed the faces of the tetrahedral cage, creating a more shielded hydrophobic cavity. A large set of potential guest molecules was screened for binding inside this hydrophobic cavity, which had an estimated volume of more than $400 \AA^{3}$. The size of the guest was found to be the major parameter dictating the binding behaviour. From a catalysis point of view, it is worthwhile to point out that the enantiopure cage was found to act as a catalyst in the hydrolysis of the pesticide and chemical warfare agents (CWA) simulant 
dichlorvos, yielding the products dimethyl phosphoric acid and dichlorovinylmethyl phosphoric acid. While the authors remain somewhat cautious in the mechanistic explanation for the catalytic acceleration, they propose the involvement of the hydroxy groups of the glyceryl moieties. The polarisation of the encapsulated dichlorvos by the positively charged cage was also suggested to facilitate nucleophilic attack at the phosphorus centre, ultimately leading to hydrolysis.

\subsection{Guest binding}

As is clear from the previous section, binding of hydrophobic guests inside of a cage cavity has enabled catalytic transformations of small molecules to proceed via pathways not normally observed in aqueous media. However in recent years, the process of guest binding in water itself has also become the subject of careful, detailed studies. Apart from non-covalent interactions that can facilitate binding, such as hydrogen bonding, ionic or aromatic interactions, which are operative in any solvent, in water the binding is often also driven by the hydrophobic effect. Consequently, host-guest binding in water can be a perplexing combination of processes driven by both enthalpy and entropy, thus warranting detailed physical chemical studies to unravel the intricacies of guest binding in water. ${ }^{[13]}$

Work by Arena, Raymond and co-workers nicely illustrates the different, and often opposing forces that lead to guest encapsulation in combination with the possibility of exterior binding. ${ }^{[71]}$ It also underlines the necessity of using a range of different analytical techniques in order to fully understand the binding process of charged guests in and around coordination cages, and thus obtain all relevant thermodynamic parameters for guest binding in aqueous solution. Arena, Raymond and co-workers employed a combination of NMR and UV/vis spectrometry as well as calorimetry to untangle the multiple binding equilibria that are established upon introduction of a cationic guest molecule into a solution of the polyanionic 
cage 2 (Figure 2). Apart from binding in the cage's internal cavity, a guest molecule could also bind to each of the four faces of the tetrahedral cage. Assuming that each binding event is independent, a total of five binding constants ( $K$ value) were needed to fully describe this system, while each $K$ value can be further broken down into an enthalpy contribution $(\Delta H)$ and entropy contribution $(\Delta S)$. By performing detailed binding studies with cationic guests of different sizes, the effect of guest size and shape on the strength of binding was first quantified. Binding of the cationic guest inside the cage's cavity was always entropy driven with $\Delta S$ values between 40 and $80 \mathrm{~J} \mathrm{~K}^{-1} \mathrm{~mol}^{-1}$ being reported. These results are in accordance with the hydrophobic effect being operative in water. In contrast, ion binding on the exterior of the cage was found to be enthalpically driven for smaller guests due to ionic, cation- $\pi$ and $\mathrm{CH}-\pi$ interactions. For larger guests desolvation of the cation, an entropy-driven process, controlled the exterior binding.

More recently the same group addressed another feature of enzyme-mediated reactions: conformational selection, the process that describes how ligand recognition can drive the equilibrium between various active protein conformations. ${ }^{[72]}$ Selective inversion recovery ${ }^{1} \mathrm{H}$ NMR studies offered mechanistic insight into how the distribution of the facecapped $\mathrm{M}_{4} \mathrm{~L}_{4} \mathrm{Ga}(\mathrm{III})$ cage $\mathbf{1 2}^{[73]}$ varied between two diastereomers (with $T$ and $S_{4}$ symmetry) in response to an externally added cationic guest molecule (Figure 6). The cationic guest was better accommodated in the larger $T$-symmetric cage, and consequently the rate at which this diastereoisomer increased was reported to be inversely correlated to the concentration of the guest molecule. The results presented by the authors are singularly consistent with conformational selection, as opposed to an induced fit model, being operative in the molecular recognition process. 
A

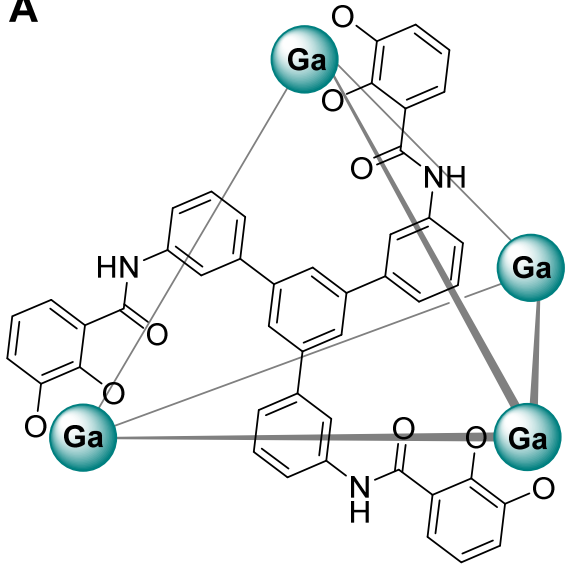

12
B

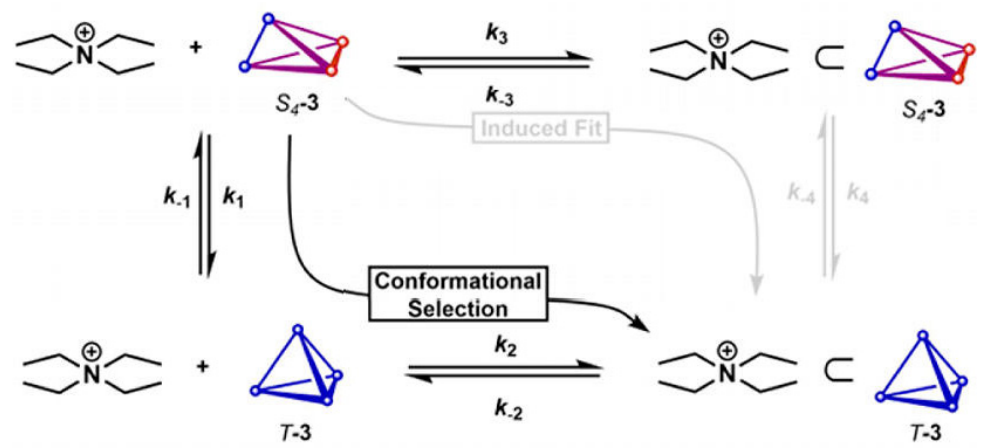

Figure 6. A: Schematic structure of the face-defining $\mathrm{M}_{4} \mathrm{~L}_{4}$-type $\mathrm{Ga}(\mathrm{III})$ cage 12. ${ }^{[73]} \mathrm{B}$ : Two possible mechanism for guest binding by cage 12: induced fit and conformational selection mechanism for encapsulation of ammonium guests; the latter was found to be operative in cage 12.

The Ward group have also reported extensive binding studies exploring how hydrophobic guests can bind in the cavity of their previously discussed, water-soluble octanuclear cubic Co(II) cage 6 (Figure 3). ${ }^{[27,74-76]}$ In their earlier work, ${ }^{[27]}$ the size and shape criteria for guest binding inside the cavity of $\mathbf{6}$ was established using a fluorescence displacement assay to quantify guest binding. To determine the size effect, aliphatic cyclic ketones of increasing size $\left(\mathrm{C}_{5}\right.$ to $\left.\mathrm{C}_{13}\right)$ were evaluated, revealing a linear relationship between the free energy, $\Delta G$, of binding and the guest's surface area for a ring size up to $\mathrm{C}_{11}$. Larger guests were found to be too large to be tightly bound. To address the shape and flexibility, a series of $\mathrm{C}_{10}$ aliphatic ketones that differed in shape, but not their size, were studied. The strongest binding guests from this series were found to be cyclic, rigid and preorganised guests. Overall the authors conclude that based on their physical chemical studies guest binding can be predicted on the basis of the correlation between $\Delta G$ and guest surface area, provided the guest is monocyclic and therefore preorganised, and small enough to fit inside the cavity in the first place. In follow-up work, binding studies were extended further to include the use of docking software that had originally been developed for analysis of 
protein-ligand interactions in drug discovery. The authors demonstrate that this software can be used to identify new guests for binding in their octanuclear Co(II) cage, ${ }^{[75]}$ as well as to systematically investigate the effect of guest flexibility. ${ }^{[76]}$

Earlier this year, the group explicitly addressed the contribution of the hydrophobic effect in the binding of guest molecules in cage $6 .{ }^{[74]}$ On the basis of crystallographic studies it was concluded that the cubic cage can accommodate a cluster of 10 water molecules, with an average number of hydrogen bonds per water molecule that is 0.5 hydrogen bonds less than in bulk solution. These "high-energy" water molecules provide a driving force for guest binding. This driving force is, however, not predominantly entropy-based, as would be the case when considering the classic hydrophobic effect; instead it is enthalpy-driven, as release of these "hydrogen-bond frustrated" water molecules increases the number of intermolecular hydrogen bonds. These insights underline that a complete understanding of guest binding in water driven by the hydrophobic effect is far from trivial to acquire. For cucurbiturils, similar observations of large enthalpic contributions to the hydrophobic effect have been reported. ${ }^{[77]}$

In an attempt to dissect the effect of guest structure on both the strength of guest binding as well as on the rate of guest uptake, Nitschke and co-workers relied on principle component analysis (PCA) to quantify the contribution of five guest descriptors to guest binding within the water-soluble, tetrahedral Fe(II) cage 13 (Figure 7). ${ }^{[78]}$ Previously, this cage was known to bind a variety of guests, ranging from simple alkanes ${ }^{[79]}$ to pyrophoric $\mathrm{P}_{4},{ }^{[57]}$ and from the potent greenhouse gas $\mathrm{SF}_{6}{ }^{[51]}$ to the reactive diene furan. ${ }^{[80]}$ To elucidate the factors governing the strength and kinetics of binding, detailed NMR studies were performed on a library of 24 structurally diverse guest molecules. From guest titrations the strength of binding could be determined. Complementary time-dependent studies provided information on the rate of guest uptake. PCA revealed that from the five molecular guest descriptors considered (the logarithm of the octanol:water partition coefficient $(\log P)$, the 
dipole moment, the molecular volume, the molecular surface area and the asphericity, which is a descriptor to what extent the guest's shape deviates form a spherical shape), the strength of binding was most strongly correlated to the $\log \mathrm{P}$ values, while the rate of binding was most strongly correlated to the shape of the molecule. These quantitative insights allowed for the design of multiple guest-binding experiments in which variations in the binding strength and rate of guest uptake were exploited to control the order of encapsulation as a function of time (Figure 7). As pointed out by the authors, the time-dependent sequential uptake and release of specific guests might be used in similar systems to control the concentrations of specific reagents in a reaction mixture, or to release one reagent specifically as another is produced to displace it.

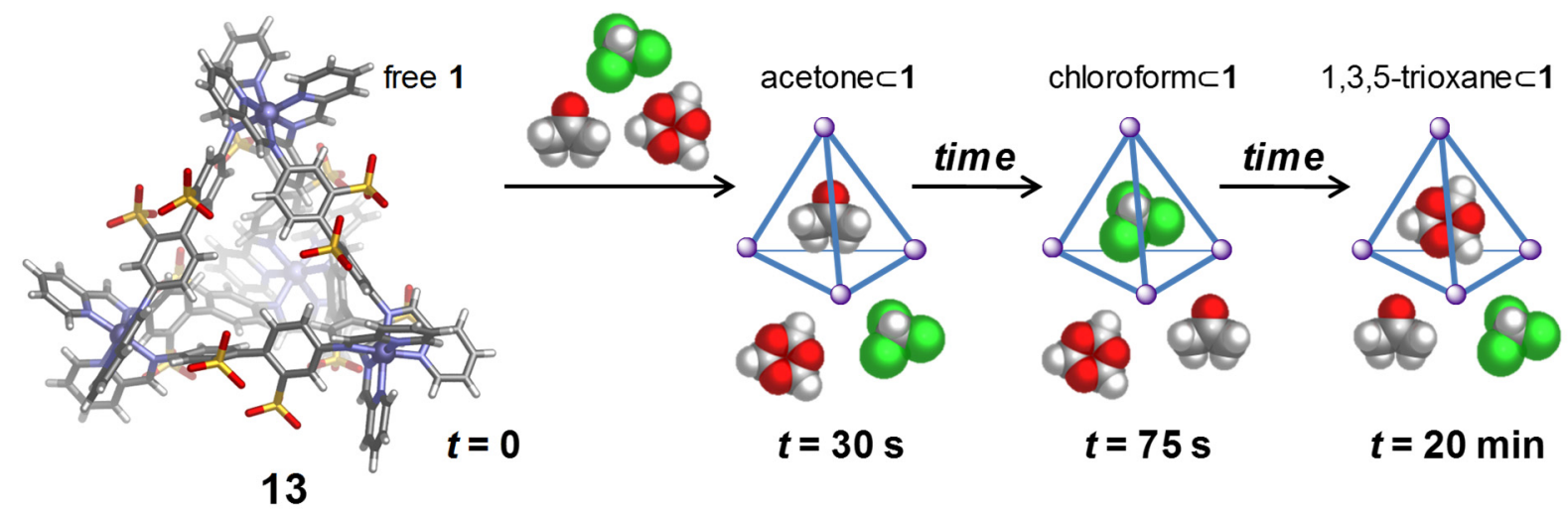

Figure 7. Sequential formation of acetone, chloroform and 1,3,5-trioxane host-guest complexes following simultaneous addition of all three guests to cage $13 .{ }^{[78]}$

\subsection{Guest delivery and controlled release}

In the previous sections examples were discussed of water-soluble cage complexes that could either simply bind a guest molecule (section 2.3), or that could bind a guest that then underwent a catalytic transformation (section 2.2). Another possibility for the encapsulated guest is for it to be released again under the right conditions and/or upon application of a desired stimulus. Considering the field of 3D metal-organic cage complexes, it can be concluded that, irrespective of solvent, the focus is primarily on guest binding, and not so much on guest release. Nevertheless, the possibility of controlled guest release is a valuable 
research objective with associated potential applications in aqueous media, where guest release can be used in the context of drug delivery. Controlled release of reactive guests is also a viable route to control chemical reactivity. In 2012 Smulders and Nitschke demonstrated how through encapsulation the water-soluble cage $\mathbf{1 3}$ (Figure 7) can prevent a reactive diene, furan, from reacting with its counterpart, maleimide, in solution. ${ }^{[80]}$ To release the reactive diene from the cage's interior, a stronger binding guest, benzene, simply needed to be added. Once released, the diene quickly reacted with the dienophile to form the DielsAlder adduct with a rate that was more than an order of magnitude higher than when it remained encapsulated. As the binding of the guest, and therefore also its reactivity, are largely determined by the size of the guest and not by its chemical functionality, the presented approach is an alternative to the "classical" protecting group strategy that relies on chemical functionality. Evidently, this strategy is however limited to hydrophobic reactants.

Yoshizawa and co-workers have reported the quantitative encapsulation and stabilisation of a radical initiator inside a polyaromatic $\mathrm{Pd}(\mathrm{II})$ nanocapsule in aqueous medium (Figure 8). ${ }^{[48]}$ The choice for rigid, poorly soluble, aromatic ligands was based on their aim to synthesise a polyaromatic cage that can strongly absorb (UV) light, allowing the photoreactivity of encapsulated guests to be manipulated. However, to maintain sufficient solubility in aqueous medium the aromatic ligand needed to be equipped with three solubilising 2-(2methoxyethoxy) side chains. Apart from enhancing the thermal stability of azo-based radical initiators, more remarkably, the cage was indeed also capable of imparting stability towards UV light: the decomposition half-life of AIBN was increased by a factor of $\sim 380$ upon encapsulation inside the polyaromatic cage. The observed stability towards light was attributed to the optical shielding by the aromatic panels that outline the cage. This explanation was supported by strong absorption band in the UV region (300-450 nm) of the UV-vis spectrum. While in water, the radical initiator could be stabilised, enabling safe 
storage, and then released from its hydrophobic cavity by solvent exchange. Solvent exchange was readily performed by evaporating the aqueous solution, and redissolving the isolated host-guest complex in toluene. In toluene, liberated AIBN was capable of initiating the thermal radical polymerisation of methyl methacrylate as well as the corresponding photopolymerisation. Both thermal and photoinduced procedures yielded comparable polymers, in terms of average chain length and polydispersity, to those produced using pristine AIBN.

A

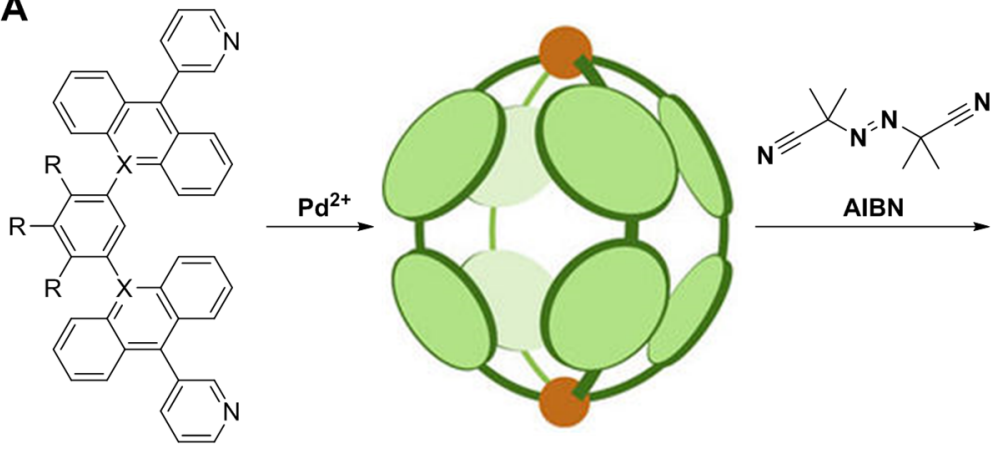

Cage 14: $\mathrm{R}=\mathrm{OCH}_{2} \mathrm{CH}_{2} \mathrm{OCH}_{3}, \mathrm{X}=\mathrm{C}$ Cage 15: $\mathrm{R}=\mathrm{H}, \mathrm{X}=\mathrm{N}^{+}$

B

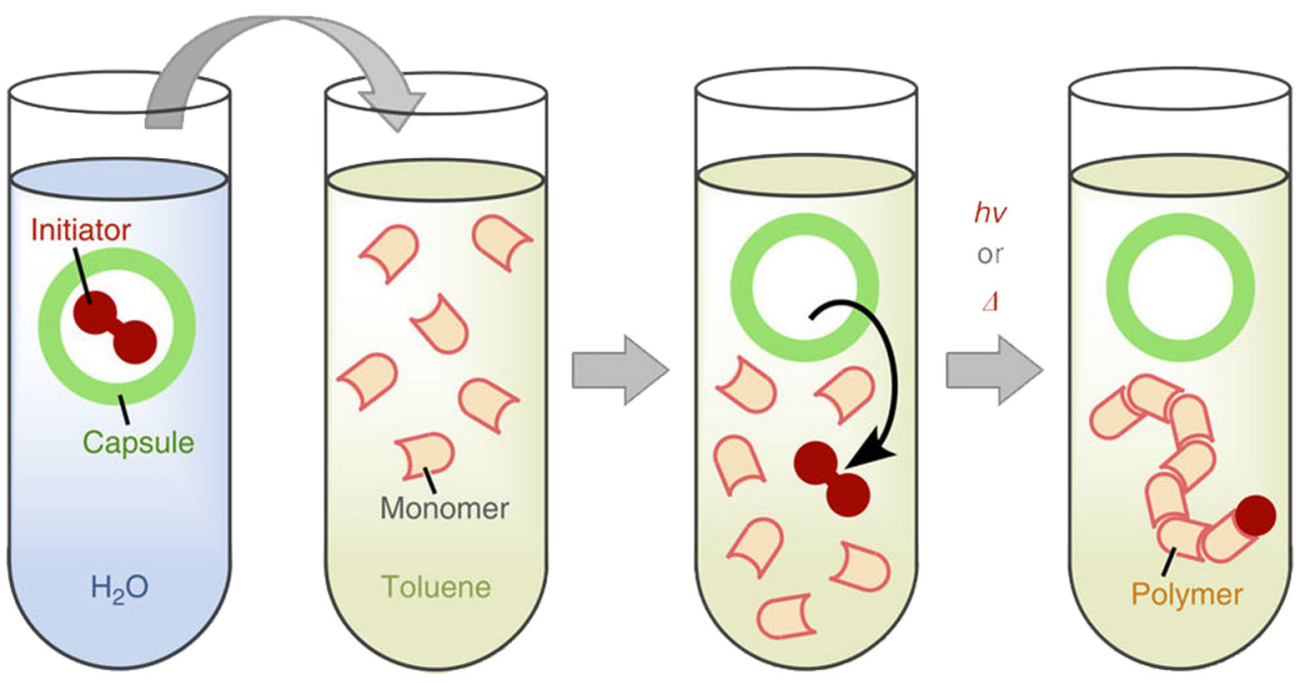

Figure 8. A: Synthesis of water-soluble polyaromatic $\operatorname{Pd}(\mathrm{II})$ nanocapsules 14 and 15 and subsequent encapsulation of AIBN (by cage 14). ${ }^{[4-49]}$ B: Addition of the encapsulated radical initiator to an organic solution containing monomers, leading to spontaneous release of the initiator from the capsule that can then initiate polymerisation of the monomer by photo or thermal stimuli. ${ }^{[49]}$ 
In their efforts to further enhance the water solubility of cage 14, the same research group replaced the neutral anthracene panels on the complex with isostructural, yet positively charged, acridinium panels (Figure 8 ). ${ }^{[49]}$ The polycationic cage generated was highly soluble in water where it displayed an unusual recognition ability for anionic organic molecules containing a trifluoroborate group.

Employing a similar approach to Yoshizawa, Shi, Su and co-workers reported a lightabsorbing heterometallic ruthenium-palladium coordination cage. ${ }^{[81]}$ Light absorption by the ruthenium phenanthroline moieties within the cage, followed by (multichannel) electron transfer from the chromophoric Ru to the catalytic Pd centres, allowed the cage to be used for photochemical hydrogen production.

There are multiple approaches to induce the release of a guest from a cage. Firstly, release can simply by triggered by changing the temperature. Depending on the nature of the binding event: whether the binding is enthalpy or entropy-driven, the temperature needs to be raised, or lowered, respectively to induce guest release. Secondly, as discussed for cage 14, changing the medium can affect the strength of binding and thereby induce guest release. Thirdly, as also discussed above for the release of furan from cage $13,{ }^{[80]}$ addition of a second, competing guest can displace the original guest from a cage. Hunter and Ward have reported another elegant approach to induce guest release from their octanuclear Co(II) cage 6, (Figure 3). ${ }^{\left[{ }^{[2]}\right.}$ In earlier work they established that guest binding by this cage in water is driven by the hydrophobic effect, meaning that hydrophobic, uncharged guest molecules are most strongly bound, while charged guests are only weakly bound as they are preferentially solvated by water. Consequently, when combined with the possibility of effortlessly changing the $\mathrm{pH}$ of an aqueous solution, this led to the research hypothesis that the degree of binding of a guest with a functional group that can be protonated (e.g. amine) or deprotonated (e.g. carboxylic acid) should be highly $\mathrm{pH}$-dependent, thus offering the possibility of $\mathrm{pH}$-controlled guest 
release. Indeed, for a range of guests with varying functional groups including primary and tertiary amines, quinoline, imidazole, and carboxylic acids, pronounced shifts in binding constants were found, as graphically summarised in the chart in Figure 9. The largest, reversible change in binding constant, from $1 \times 10^{4} \mathrm{M}^{-1}$ in the neutral form to $13 \mathrm{M}^{-1}$ in the positively charged state was observed for 1-aminoadamantane. Finally, within the context of drug delivery in water, it should be pointed out that some of the reported guests are biologically active: adamantine (1-aminoadamantane - a drug to treat Parkinson's disease as well as an anti-viral agent to treat influenza), nicotine and aspirin could all be released from the cage's cavity by an appropriate change in $\mathrm{pH}$.

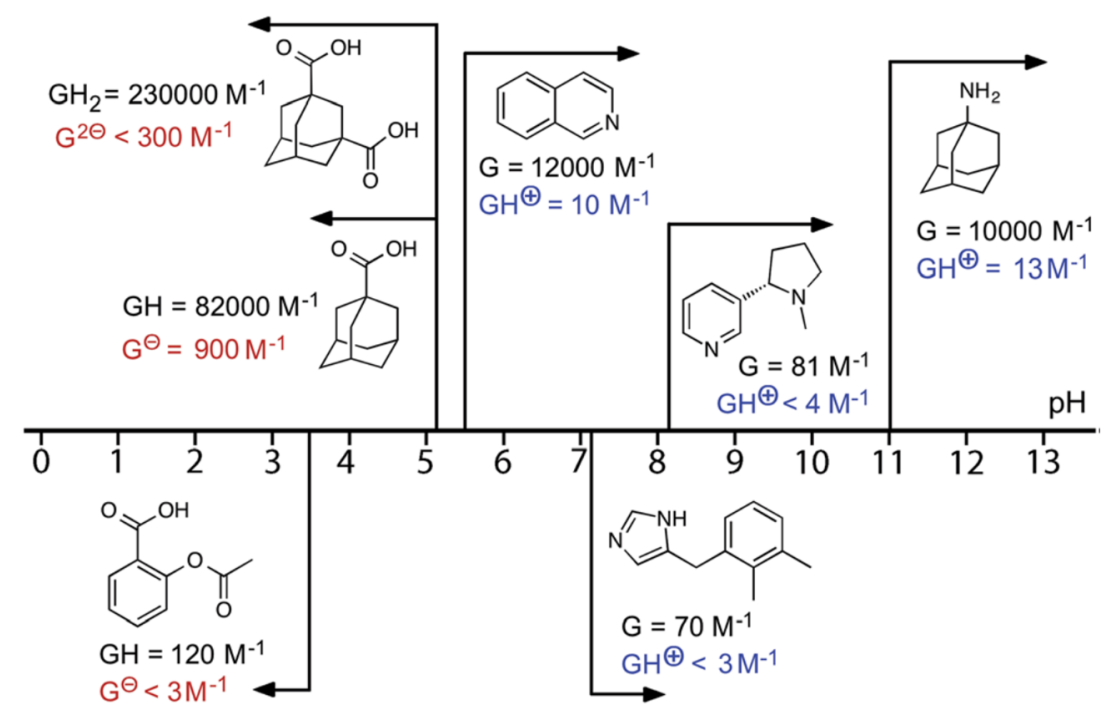

Figure 9. Graphical summary of association constants for guests in neutral and charged states that can be bound in, and released from, cage $\mathbf{6}$, by control over $\mathrm{pH} .{ }^{[82]}$

The group of Therrien has significantly developed the use of ruthenium-based cage complexes for biological applications, making use of both the compatibility of areneruthenium complexes with water, and of the fact that the commonly found +2 oxidation state of ruthenium is often the preferred oxidation state of biologically active ruthenium species in vivo. ${ }^{[83]}$ As an approach to non-covalent drug delivery the authors present the Trojan Horse approach: ${ }^{[84-85]}$ This approach relies on the anticancer activity of arene-ruthenium complexes that are water-soluble, in combination with the excellent stability of arene-ruthenium-based 
cages that can encapsulate biologically active guest molecules that impart additional features to the system (selectivity, sensing, targeting, toxicity). Moreover encapsulation of bio-active guest molecules not only enhances their water solubility (when the guest is hydrophobic), but can also facilitate internalisation of the bio-active guest within cancer cells, allowing the $\mathrm{Ru}$ cages to operate as drug-delivery vectors.

A

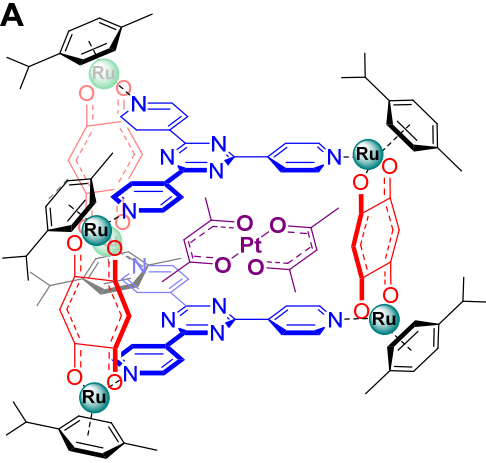

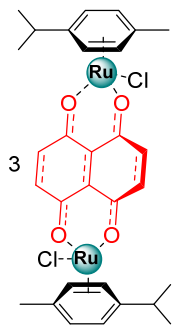

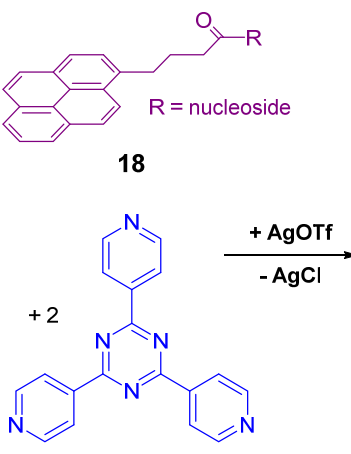

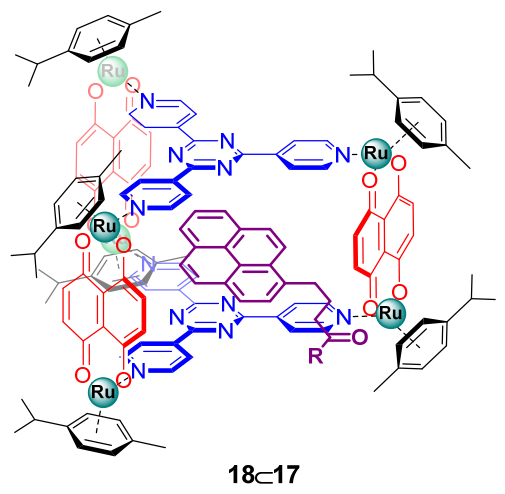

Figure 10. A: Molecular structure of first-generation Ru cage 16 that can encapsulate and deliver Pt(II) complexes. B: Synthesis of a larger, second-generation Ru cage 17, that can bind and release synthetic nucleosides (indicated by R) tethered to a hydrophobic pyrene group 18. ${ }^{[86]}$

In their earlier work on the Trojan Horse concept, Therrien and co-workers demonstrated the increased cytotoxicity of $\mathrm{Pd}(\mathrm{II})$ and $\mathrm{Pt}(\mathrm{II})$ complexes encapsulated in cage 16 (Figure 10A) to human A2780 ovarian cancer cells. ${ }^{[85]}$ Extending this research, Therrien, Kim and co-workers subsequently demonstrated that a larger Ru cage (cage 17, Figure 10B) was capable of encapsulating synthetic nucleosides with known antiviral and anticancer properties. ${ }^{[86]}$ To this end, the nucleoside was appended with a hydrophobic pyrene moiety. The authors demonstrated that their encapsulation strategy greatly increased the water solubility, and more importantly, the cytotoxicity with respect to the empty cages. The same $\mathrm{Ru}$ cages, have also been shown to encapsulate, and subsequently deliver, hydrophobic porphyrin molecules to cancer cells. ${ }^{[87]}$ Following internalisation, the photosensitiser porphyrin was then photoactivated, leading to a significant increase in cell death. In summary, 
the water-soluble Ru cage provides a vehicle for drug delivery in aqueous medium, overcoming the poor cellular uptake and bioavailability of hydrophobic drug candidates.

As an alternative approach to the non-covalent drug delivery approach reported by Therrien, Isaacs and co-workers reported the surface functionalisation of a large $\mathrm{Pd}_{12} \mathrm{~L}_{24}$ architecture with methyl viologen units capable of non-covalently binding CB8 and doxorubicin conjugated 2-alkoxynaphthalene (DOX-R) in a rotaxane arrangement (Figure 11). ${ }^{[88]}$ Despite the parent $\operatorname{Pd}_{12} \mathrm{~L}_{24}$ structure (19) being water insoluble, formation of the ternary complex occurred in water, enabling $\mathbf{1 9}$ to act as a non-covalent drug delivery vehicle. Cellular uptake and cytotoxicity studies performed in HeLa cells supported increased uptake of the ternary complex versus $\mathbf{D O X}-\mathbf{R}$, results that correlated with a reduction in cellular viability at comparable doxorubicin concentrations. The size, charge and ordered threedimensional rotaxane arrangement of the ternary complex is attributed to the favourable cell uptake and cytotoxicity profile, and is expected to have further benefits with regard to circulation time and tumour accumulation in in vivo treatments. The water stable non-covalent loading of 19 thus represents a novel application for water-soluble self-assemblies.

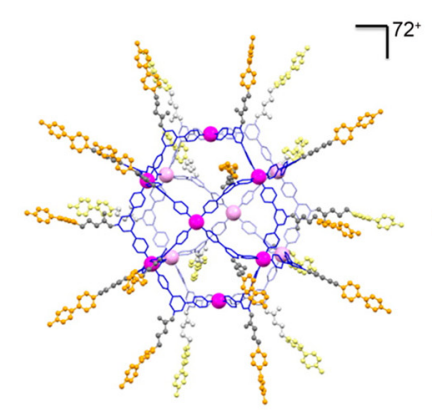

$\left[\mathrm{Pd}_{12} \mathrm{~L}_{24}\right]^{72+}$

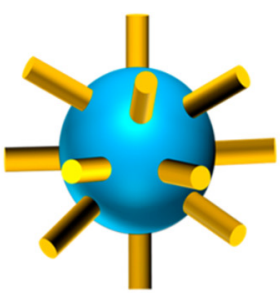

19

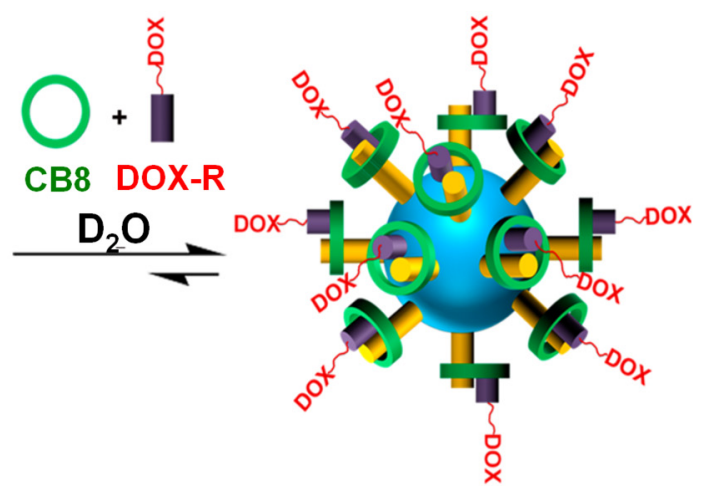

$\left[\mathrm{Pd}_{12}(\mathrm{~L} \cdot \mathrm{CB} 8 \cdot \mathrm{DOX}-\mathrm{R})_{24}\right]^{72+}$<smiles></smiles>

Figure 11. A non-covalent drug delivery strategy: self-assembly of water-soluble $\left[\mathrm{Pd}_{12}(\text { L.CB8.DOX-R })_{24}\right]^{72+}$ from water insoluble 19 and doxorubicin conjugated 2alkoxynaphthalene (DOX-R). Doxorubicin is a clinically approved organic cancer drug. 
Lippard and co-workers reported encapsulation and delivery of a $\mathrm{Pt}(\mathrm{IV})$ prodrug using an octahedral $\mathrm{Pt}(\mathrm{II})$ cage $\mathbf{2 0}^{[89]}$ that had previously been reported by Fujita. ${ }^{[90]}$ The $\mathrm{Pt}(\mathrm{IV})$ prodrug was tethered to a large hydrophobic adamantane group that directed the prodrug encapsulation (Figure 12). The size of the hydrophobic cavity allowed for the simultaneous encapsulation of four adamantane units from prodrug guest molecules. The net positive charge of the complex promoted its cellular uptake, and once inside the cell, biological reductants, including ascorbic acid, were postulated to induce release of the active anticancer agent, cisplatin (Figure 12), ultimately causing cell cycle arrest and apoptosis. Gratifyingly, the complex displayed cytotoxicity on a par with cisplatin against a panel of human cancer cell lines. Equivalent cytotoxicity was not achieved by the cage or prodrug alone.

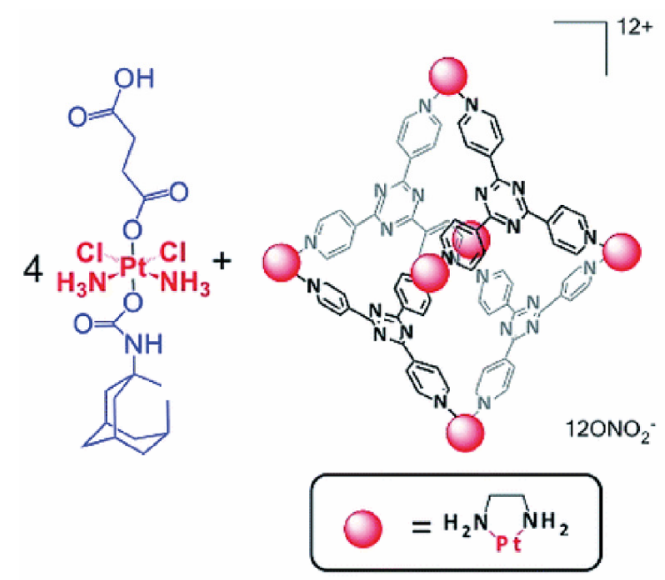

20

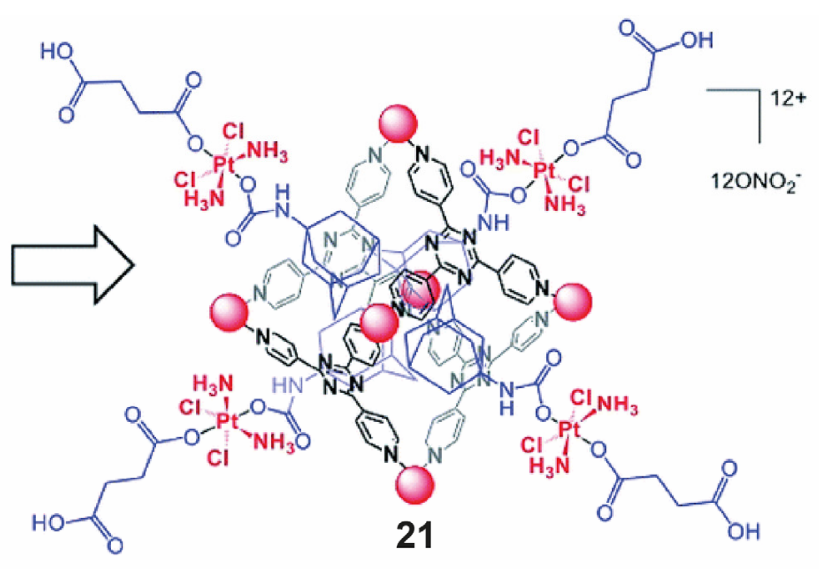

Ascorbic Acid
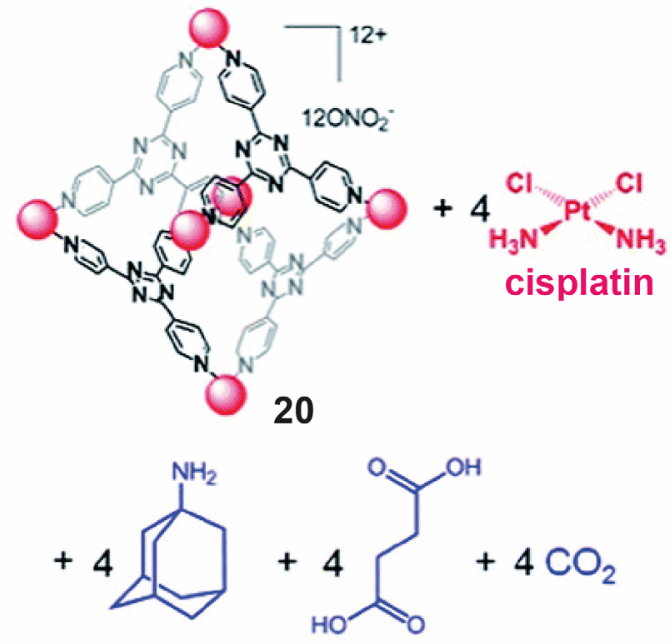

Figure 12. Encapsulation of the $\mathrm{Pt}(\mathrm{IV})$ prodrug 19 in cage 20, and subsequent release induced by reduction with ascorbic acid. ${ }^{[89]}$ 


\subsection{Guest-induced host transformation}

So far, our discussion has focussed on how a cage can act as a host for a guest molecule, with focus on simple guest binding, controlled release mechanisms and applications to catalysis. Implicitly it was assumed that the host during this process remains more or less constant in size and shape. In fact, the cage's rigidity and permanent shape can impart size and/or shape selectivity on any reaction catalysed by the cage (vide supra). Provided, however, the transition metal used is sufficiently labile, a coordination cage may be converted into one or more alternative cage complexes. Recent studies by Cullen, Hunter and Ward have explored the effect of various parameters (temperature, concentration and solvent) on the interconversion of three $\mathrm{Co}(\mathrm{II})$ complexes in water: a $\mathrm{Co}_{2} \mathrm{~L}_{3}$ helicate, a tetrahedral $\mathrm{Co}_{4} \mathrm{~L}_{6}$ cage and a dodecanuclear truncated-tetrahedral $\mathrm{Co}_{12} \mathrm{~L}_{18}$ cage ${ }^{[91]}$ Interestingly, the hydrophobic effect was attributed as the driving force for the interconversion from the smallest to largest complex. Despite the largest construct being entropically disfavoured, it is observed as the reorganisation of several smaller complexes into one larger one results in a smaller proportion of the hydrophobic ligand surface being exposed to water, with a larger proportion of the ligand surface being protected in the interior of the assembly.

In addition, guest encapsulation can also induce a transformation from one coordination complex to another; an established example being the guest-templated conversion from a $\mathrm{M}_{2} \mathrm{~L}_{3}$ helicate to a tetrahedral $\mathrm{M}_{4} \mathrm{~L}_{6}$ host. ${ }^{[92]}$ More recently, Fujita and coworkers have reported how hydrophobic guest binding could both induce the transformation of a water-soluble bipyramidal $\mathrm{M}_{18} \mathrm{~L}_{6}$ capsule into a larger octahedral $\mathrm{M}_{24} \mathrm{~L}_{8}$ cage ${ }^{[93]}$, as well as the transformation between an octahedral $\mathrm{M}_{20} \mathrm{~L}_{8}$ cage and bowl-shaped $\mathrm{M}_{8} \mathrm{~L}_{4}$ cage. ${ }^{[94]}$

Last year the Nitschke group reported a more subtle example where anion binding induced the transformation of one tetrahedral $\mathrm{Fe}_{4} \mathrm{~L}_{4}$ cage into an isomeric tetrahedral $\mathrm{Fe}_{4} \mathrm{~L}_{4}$ cage (Figure 13). ${ }^{[95]}$ Their face-defining ligand was built from cationic azaphosphatrane-based 
subcomponents, which imparted water solubility and promoted anion binding. The azaphosphatrane is a non-planar, concave ligand, and studies revealed that the tetrahedral $\mathrm{Fe}$ cage could either be assembled with all four azaphosphatrane faces "bulging out" (resulting in a $T$-symmetric cage), or with one of the four faces "bulging in" (leading to a $C_{3}$-symmetric cage). The equilibrium between these two isomers could be controlled by the size of the anion that could be bound inside the cage's cavity, making use of the cationic ${ }^{+} \mathrm{P}-\mathrm{H}$ moiety to bind anionic guests (Figure 13).This "bulging-in effect", which was mediated by detachment of the iminopyridine moieties from metal, caused a dramatic decrease in cavity volume from $219 \AA^{3}$ to $35 \AA^{3}$, as inferred from the volume of the anionic guests that could bind in the respective cages. This example shows how the dynamic nature of these water-soluble cationic cages can cause structural adaptions driven by anion binding that is mediated by electrostatic interactions between host and guest.
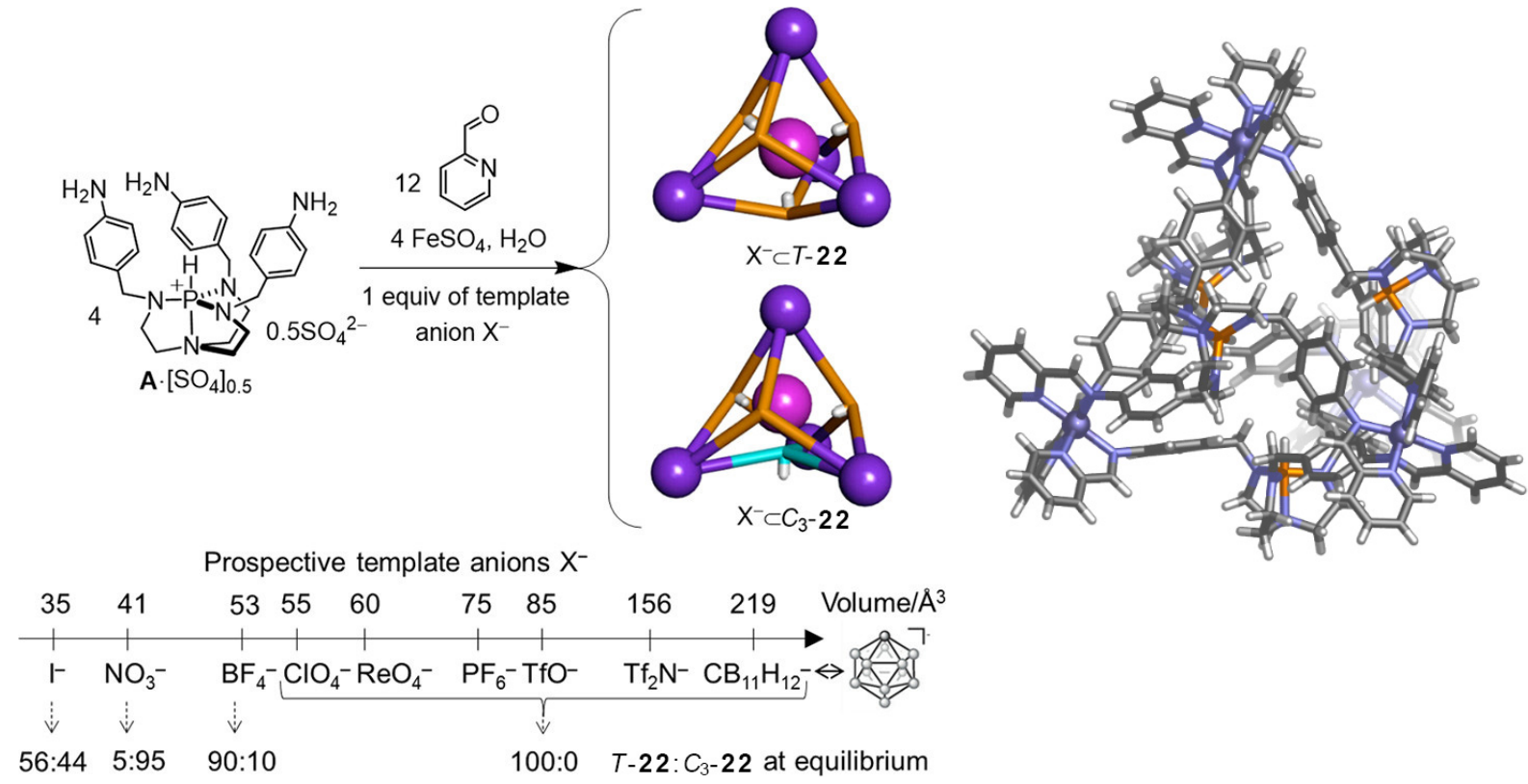

Figure 13. Schematic representation of the synthesis of the two cage isomers $T-22$ and $C_{3}-\mathbf{2 2}$, obtained by aqueous self-assembly in the presence of a template anion. The cage faces coloured orange represent endo-azaphosphatrane ligands, with ${ }^{+} \mathrm{P}-\mathrm{H}$ pointing inward, while the cyan face represents exo ${ }^{+} \mathrm{P}-\mathrm{H} .{ }^{[95]}$ The single crystal X-ray structure of the $T$-symmetric cage $T-22$ is also shown. 


\subsection{Bioactive $\mathrm{M}_{2} \mathrm{~L}_{3}$ complexes}

In contrast to the three-dimensional architectures just covered, $\mathrm{M}_{2} \mathrm{~L}_{3}$ helicates and flexicates consisting of two metal ions and three or four bridging ligands, typically do not have any significant internal binding space. ${ }^{[96-100]}$ Despite this, metal-organic helicates and flexicates, have shown promising medicinal properties, in some cases attributed to their ability to bind strongly to DNA as a result of their complementary size and shape. Like many inorganic complexes with demonstrable medicinal properties, biological studies with helicates and flexicates are frequently performed in aqueous solutions containing trace DMSO or ethanol, and solubility in pure aqueous solution has -to the best of our knowledge- not been reported. Demonstrating the stability of the complex in aqueous medium should however be a stringent requirement before assigning a metal-organic complex as a biologically active species.

Flexicates are defined as distinct from helicates as they do not possess a rigid linker that mechanically couples the chirality at the metal centres. Instead they employ flexible linkers in combination with stereochemically programmed metal centres to generate diastereomerically pure complexes. ${ }^{[101]}$

The initial impetus for development of small dinuclear complexes as novel potential drug candidates was born from modelling studies performed by Hannon and co-workers that indicated that a $\mathrm{Fe}_{2} \mathrm{~L}_{3}$ helicate (23; Figure 14A) was the correct size and shape to bind within the DNA minor groove. ${ }^{[99]}$ The comparison in size between the dinuclear complexes and small molecule complexes typically investigated in cancer research was also made, with $\mathbf{2 3}$ being more comparable to zinc finger proteins than the typical mononuclear complexes investigated. 
A

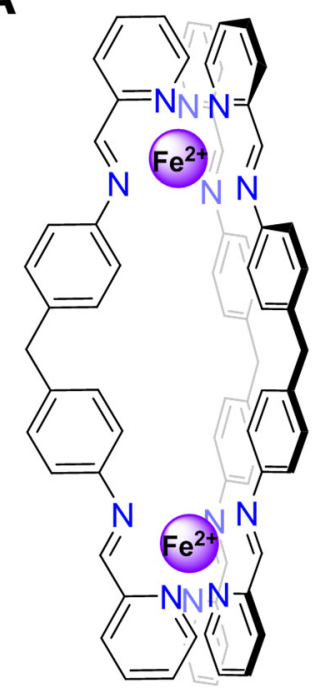

23

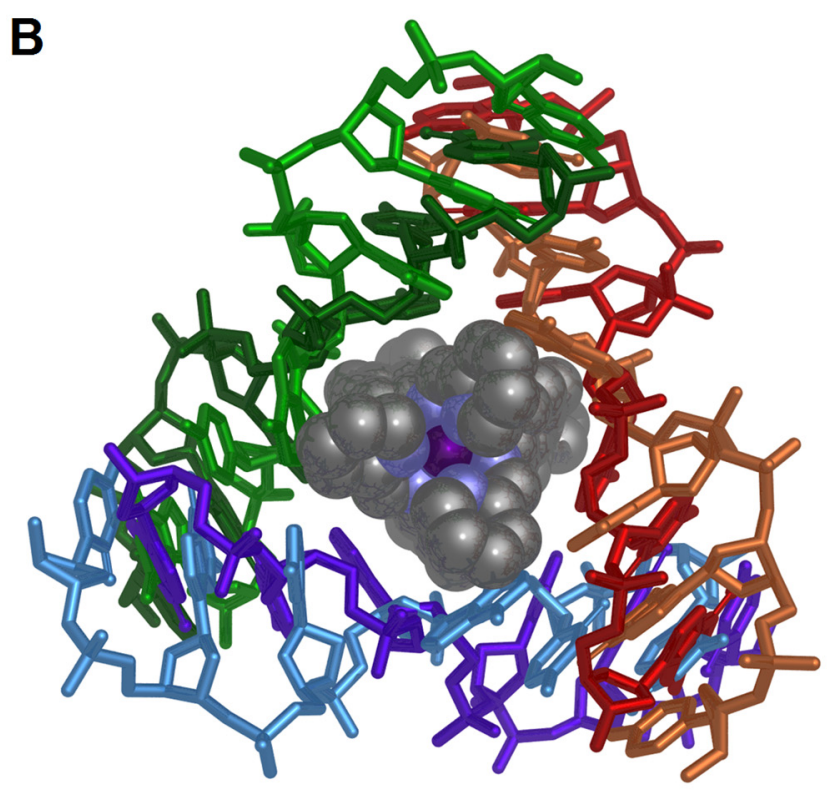

Figure 14. A: The $\mathrm{Fe}_{2} \mathrm{~L}_{3}$ helicate (23) observed to bind at the centre of a DNA three-way junction. B: Single crystal X-ray structure of $\mathbf{2 3}$ bound at the centre of three double-stranded DNA sequences. Complex $\mathbf{2 3}$ is shown as a space filling representation, DNA depicted in stick mode with individual strands differentiated by colour.

Early studies unexpectedly revealed that $\mathbf{2 3}$ crystallised at the centre of Y shaped three-way junctions generated from short oligonucleotides containing central TA sequences (Figure 14B). ${ }^{[102]}$ Examination of the crystal structure revealed that the three-fold symmetry of the helicate was coincident with that of the three-fold symmetry of the DNA junction, and no distortion in the DNA junction or the helicate structure was observed compared with single-crystal structures of the components reported in the absence of each other.

Modification of $\mathbf{2 3}$ has since produced helicates that display enhanced recognition of threeway junctions. ${ }^{[103]}$ Detailed research has also implicated $\mathrm{M}_{2} \mathrm{~L}_{3}$ complexes in selective $\mathrm{G}$ quadruplex binding, ${ }^{[104]}$ and at DNA bulges. ${ }^{[105-106]}$ A number of studies have highlighted that due to the polycationic charge of many metal-organic complexes, they are efficiently able to condense polyanionic DNA and inhibit natural enzymatic processes. ${ }^{[99,107]}$

The mode of action of these small metal-organic constructs is however not limited to DNA interactions, and biophysical measurements have shown that some flexicates do not 
interact significantly with DNA. These complexes may still show excellent anticancer properties. ${ }^{[108]}$ They were also proposed as a potential Alzheimer's treatment ${ }^{[109-110]}$ following the discovery that flexicates can interact with amyloid peptides and inhibit their aggregation.

\section{Architectures based on natural materials}

In contrast to the synthetic materials discussed thus far, biologically derived materials benefit from their inherent aqueous solubility and biocompatibility. Instead, the challenges in utilising biomaterials for aqueous and biological applications have been in manipulation, purification and characterisation of the materials. Initial development in this area focused on the design and utilisation of DNA architectures, which have high aqueous solubility due to the polyanionic, phosphate ester backbone, and can be systematically programmed through the well-understood complementary Watson-Crick base pairing between nucleotides. More recently, peptide-based architectures have also emerged as a promising platform to design water-soluble functional assemblies.

\subsection{DNA architectures}

\subsubsection{Design of DNA architectures}

The high sequence specificity of DNA has long been recognised to present a unique opportunity to rationally design water-soluble polyhedra and extended networks capable of incorporating guest molecules. ${ }^{[111]}$ However, the first literature publication detailing the construction of a discrete 3D DNA nanostructure, a DNA cube, ${ }^{[20]}$ wasn't reported until 1991, almost a decade after it was proposed. This seminal work by Seeman ${ }^{[20,112]}$ has since paved the way for extensive research in the field of DNA nanotechnology that has resulted in 
construction of ever more complex architectures, with applications ranging from nanopatterning, ${ }^{[113]}$ to protein encapsulation ${ }^{[21]}$ and drug delivery ${ }^{[114]}$ (vide infra).

In contrast to the synthetic architectures discussed in section 2, where inclusion of transition metals readily generates well-defined vertices, development of methodologies enabling well-defined vertices to be generated with DNA was less straightforward. A number of routes for formation of DNA vertices have been explored, some of which utilise only DNA while others also employ synthetic components. In 2005, Turberfield and co-workers reported a significant improvement in the synthesis of DNA architectures with publication of a protocol that enabled almost quantitative $(95 \%)$ formation of a tetrahedron from four strands of DNA (Figure 15A) in a one-step reaction that involved first heating the reaction mixture at $95{ }^{\circ} \mathrm{C}$ and then cooling to $4{ }^{\circ} \mathrm{C} \cdot{ }^{[115]}$ This synthetic protocol has since been expanded to enable synthesis of DNA tetrahedra with one edge containing a central hairpin loop sequence (Figure $15 B) \cdot{ }^{[116]}$ Such structures are able to undergo controlled expansion and contraction along one edge in response to the introduction of complementary nucleotide sequences making such assemblies highly promising candidates as environmentally responsive delivery vehicles in applications such as drug delivery. ${ }^{[117]}$

A

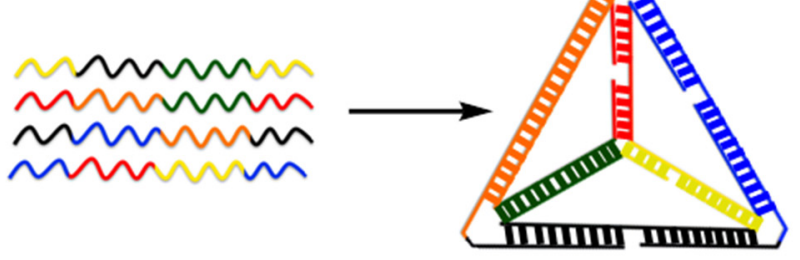

B

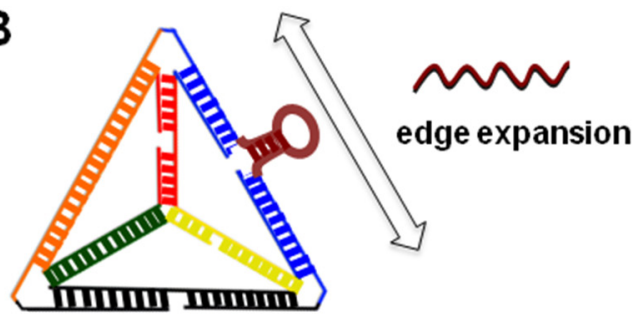

Figure 15. A: Programmed self-assembly of a DNA tetrahedron from four DNA strands and; B) a DNA tetrahedron containing a hairpin loop (maroon) within one edge. In response to a complementary oligonucleotide this hairpin loop will linearise altering the dimensions of the binding pocket at the centre of the tetrahedron.

An alternative approach to generating vertices in DNA architectures, utilising metal coordination vectors was reported independently by Sleiman ${ }^{[118]}$ and Han. ${ }^{[119]}$ Both 
publications leverage well-defined coordination motifs, ruthenium(II) tris(bipyridine) and iron(II) bis(terpyridine), respectively, to spatially orientate single-stranded oligonucleotides that can hydrogen bond with complementary sequences to generate discrete structures. Benefits of these hybrid, synthetic-DNA architectures include the high degree of structural control provided through the metal coordination geometry (see Section 2.1), the ease of preparing the constructs in an automated fashion utilising solid state methodologies commonly employed for DNA preparation, and the solubility imparted by the oligonucleotide linkers. Additional functionality, including redox or photochemical activity, may also be incorporated through the appropriate choice of metal.

Metal-free, abiological linkers have also been used to generate $3 \mathrm{D}$ architectures. ${ }^{[120-}$ ${ }^{122]}$ A 2007 report by Sleiman and co-workers ${ }^{[120]}$ represents a significant advance in this area. The publication detailed the preparation and characterisation of six new 3D architectures, more than had been reported in the preceding fifteen years, and also provided the first example of dynamically reconfigurable structures. The polyhedra were built up from twodimensional macrocycles generated from a single strand of circular DNA combined with the appropriate number of ligands, 24 (Figure 16A), containing rigid bend angles to generate a triangle, square, pentagon, or hexagon (Figure 16B). Combining the macrocycles with additional linker and rigidifying strands of DNA, generated prismatic architectures based on the parent macrocycles. This same approach was employed by the group in 2009 to generate triangular macrocycles and prisms containing bisphenanthroline binding sites at the vertices that could be metallated following assembly of the prism (Figure 16C). ${ }^{[123]}$ This postassembly metallation approach overcame limitations associated with non-specific metal binding and cleavage of DNA that had impeded development of metallated DNA architectures to that point. A range of metals were successfully incorporated within the welldefined metal binding sites, including $\mathrm{Ag}(\mathrm{I}), \mathrm{Cu}(\mathrm{I}), \mathrm{Zn}(\mathrm{II}), \mathrm{Co}(\mathrm{II}), \mathrm{Cd}(\mathrm{II}), \mathrm{Au}(\mathrm{I})$ and $\mathrm{Eu}(\mathrm{II})$. In 
addition to providing a promising route to generation of stimuli-responsive DNA

architectures, metallation of the trigonal prismatic cage significantly stabilised the

architecture, inhibiting both chemical and thermal denaturation.

A<smiles>CCCCOP(=O)([O-])Oc1ccc(-c2cccc(-c3ccc(OP(=O)([O-])O[Na])cc3)c2)cc1</smiles>

B
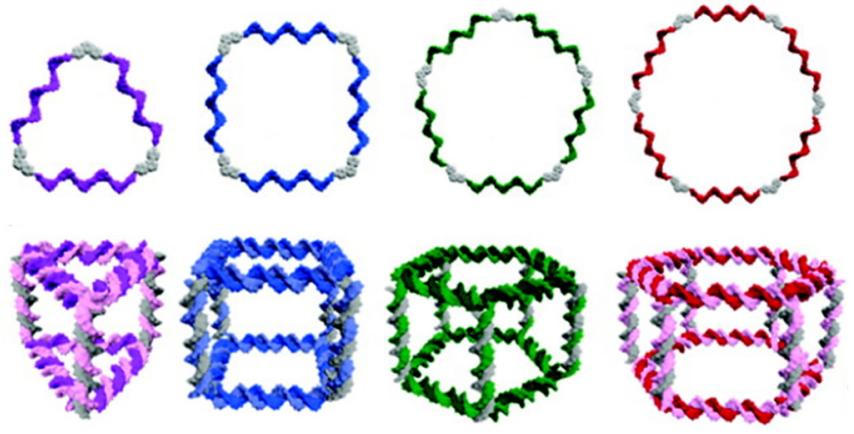

C

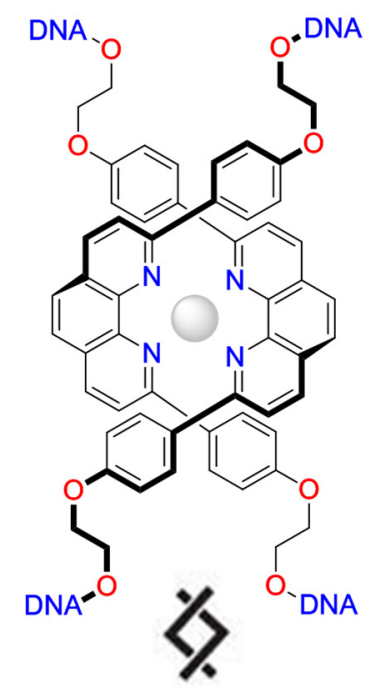
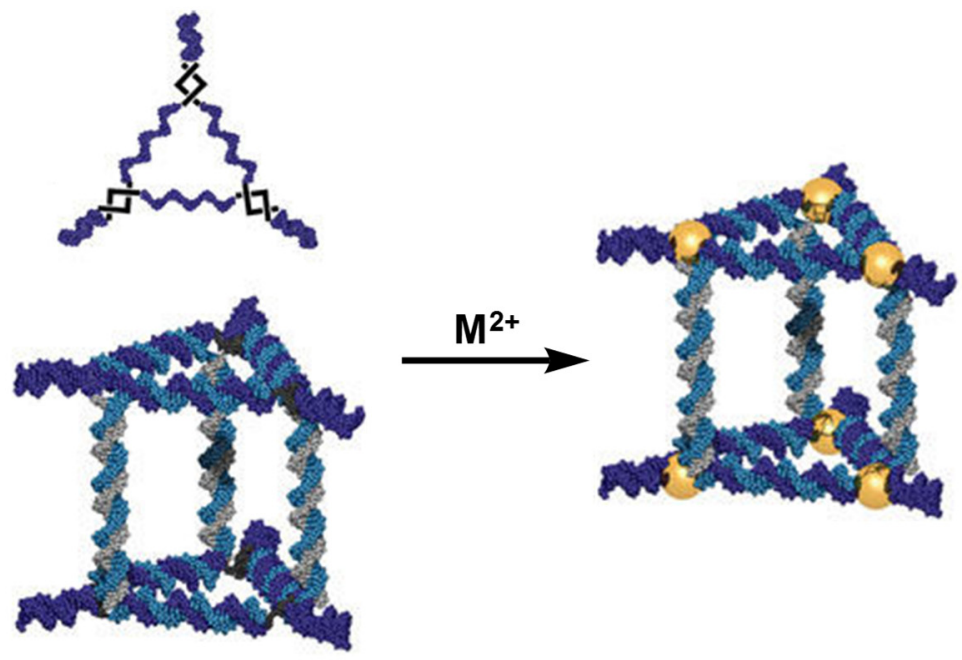

Figure 16. Construction of hybrid synthetic-DNA architectures with well-defined 3D structures. A: Ligand 24 employs phosphonate esters to tether the non-natural aromatic linker with the DNA strands; B: Combining 2D macrocycles comprised of a cyclic DNA molecule and the appropriate equivalents of $\mathbf{2 4}$ with linker and rigidifying DNA strands generates prismatic structures based on the 2D parent structures. C: Alternatively, bisphenanthroline moieties may be used to generate circular helicates that can be combined with short oligonucleotides to generate trigonal prismatic constructs capable of being subsequently metallated.

In 2008 Kiedrowski and co-workers reported the synthesis and characterisation of a dodecahedron from 20 trisoligonucleotides containing central aromatic linkers. ${ }^{[122]}$ Inclusion of the abiological aromatic linker prevented enzymatic amplification strategies for the trisoligonucletide being explored, however the authors report a solid phase synthesis strategy 
that enabled nanomolar concentrations of the purified linker to be obtained. The selfassembled dodecahedron was shown to be resistant to nuclease digestion unlike isolated dodecahedron fragments that were readily observed, by gel electrophoresis, to break down following exposure to the nuclease. This increased resistance to nuclease digestion is postulated to be due to cooperative structural integrity. In the same publication the authors report dodecahedra with extended oligonucleotide arms capable of binding up to six complementary nucleotides with fluorescein labels, thus demonstrating the potential utility of a functionalised DNA dodecahedron for multimodular binding.

Several approaches to the design and synthesis of well-defined 3D DNA architectures that involve only synthetic DNA have also been reported. ${ }^{[25,124-125]}$ DNA origami is a wellknown approach that involves the generation of face-capped architectures from a long strand of DNA (typically 1000's bp) and several short (200-300 bp) staple strands. This approach has been used to generate face-capped polyhedra including tetrahedra, ${ }^{[126]}$ and more recently expanded to the generation of structures with curvature. ${ }^{[127]}$ Architectures generated using this approach have small apertures, which is good for retaining encapsulated cargo but limits guest and/or substrate access and egress. Alternatively, framework architectures can be synthesised in which a single strand of DNA is carefully folded along the edges of an architecture, ${ }^{[128]}$ akin to the edge-bridged structures often generated from synthetic building blocks. Synthesis of framework architectures has also been demonstrated through the controlled and rationally designed combination of multiple basic DNA building units. ${ }^{[21]}$

Synthesis of DNA polyhedra from branched DNA motifs has been demonstrated by Mao and co-workers to be a robust, and rational approach to the synthesis of polyhedra frameworks with open faces. Initial studies with the three-point star motif (Figure 17), which is constructed from seven DNA single strands that combine to generate three interconnected four-arm junctions with sticky ends, indicated that the balance between flexibility and stress 
was critical if these constructs were to be able to self-assemble to form discrete architectures. ${ }^{[129]}$ Later studies confirmed that, provided all junctions faced the same way, discrete structures could be isolated when the concentration of the self-assembly reaction mixture and the number of nucleotides in the central loops was appropriately chosen. The number of nucleotides in the central loop of the junction was shown to modulate the overall flexibility of the junction, and when optimised promoted the formation of smaller $3 \mathrm{D}$, rather than 2D, structures. This rationalisation enabled the design and self-assembly of DNA tetrahedra, dodecahedra and buckyballs, all of which were characterised by DLS, AFM and cryo-EM techniques. ${ }^{[130]}$ Subsequent research has focused on construction of higher order junctions; four, ${ }^{[131]}$ five ${ }^{[132]}$ and six-point ${ }^{[133]}$ stars have been reported, and the role of symmetry on the outcome of self-assembly reactions. ${ }^{[134]}$

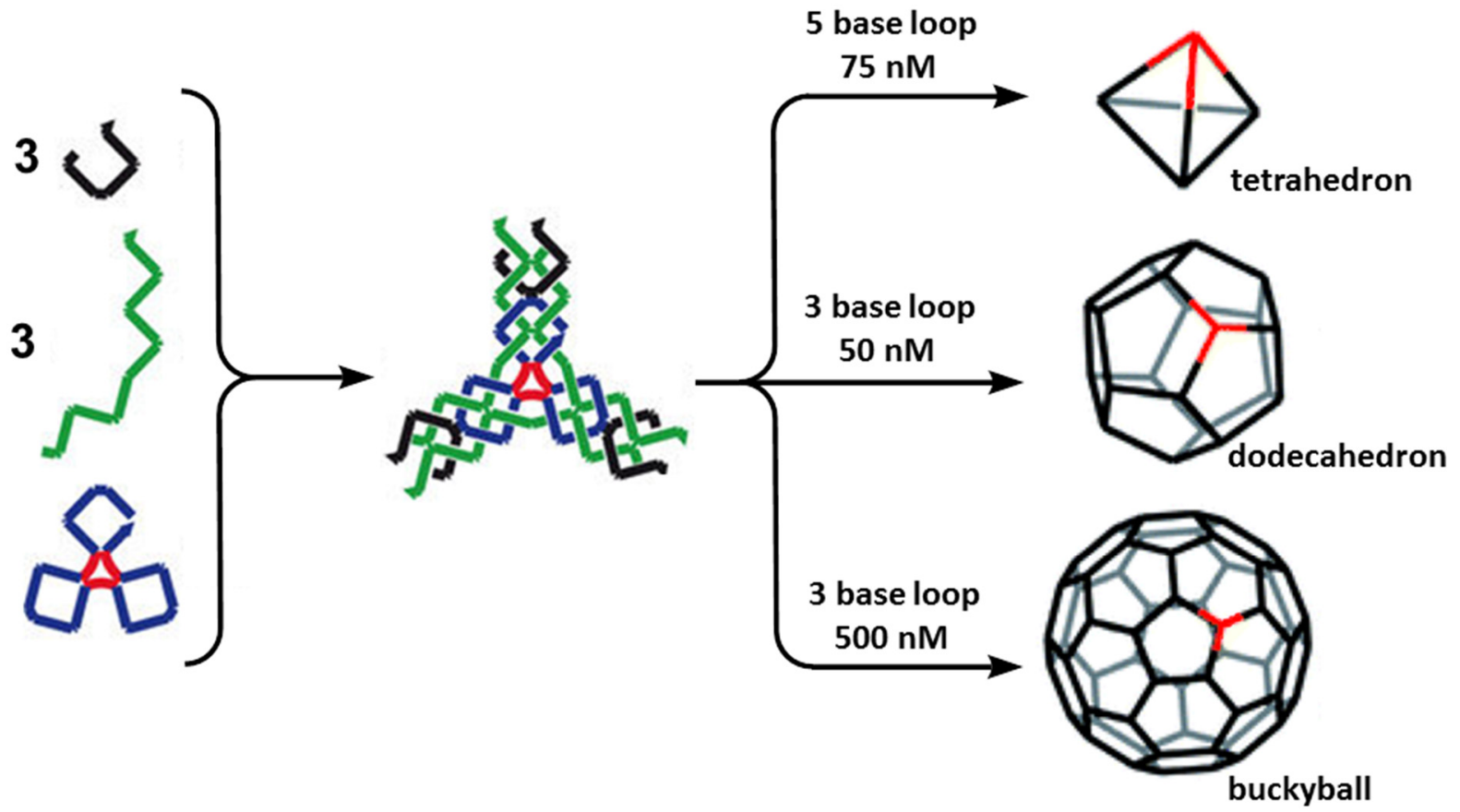

Figure 17. DNA junctions can be used to create a variety of polyhedral architectures. Key factors governing the choice of structure include the DNA concentration, the curvature and number of points on the junction and also the number of bases in the central loop. 
Recently, Sleiman and co-workers have reported on the hierarchical self-assembly of hybrid DNA cubes functionalised with synthetic, organic polymer chains (Figure 18). ${ }^{[135]}$ The length and polymer sequence, ${ }^{[136]}$ as well as the positioning of the chain on the cube was shown to have a drastic effect on the outcome of the assembly formed. In all cases the hydrophobic effect governs the assembly of the architectures in water. Alkyl chains were shown to either associate in an intramolecular manner, binding at the centre of a DNA cube generating a monomer capable of encapsulating hydrophobic molecules such as the tyrosine kinase inhibitor Dasatinib, or associate in an intermolecular manner to generate hierarchical assemblies. This hybrid structure, a DNA cube with a hydrophobic binding cavity, represents a rare example of a complex derived from biological components that is capable of utilising the hydrophobic effect to drive guest binding. Future studies exploring whether, analogously to the purely synthetic constructs discussed in Section 2, this family of complexes can perform catalysis and controlled released, will prove insightful.

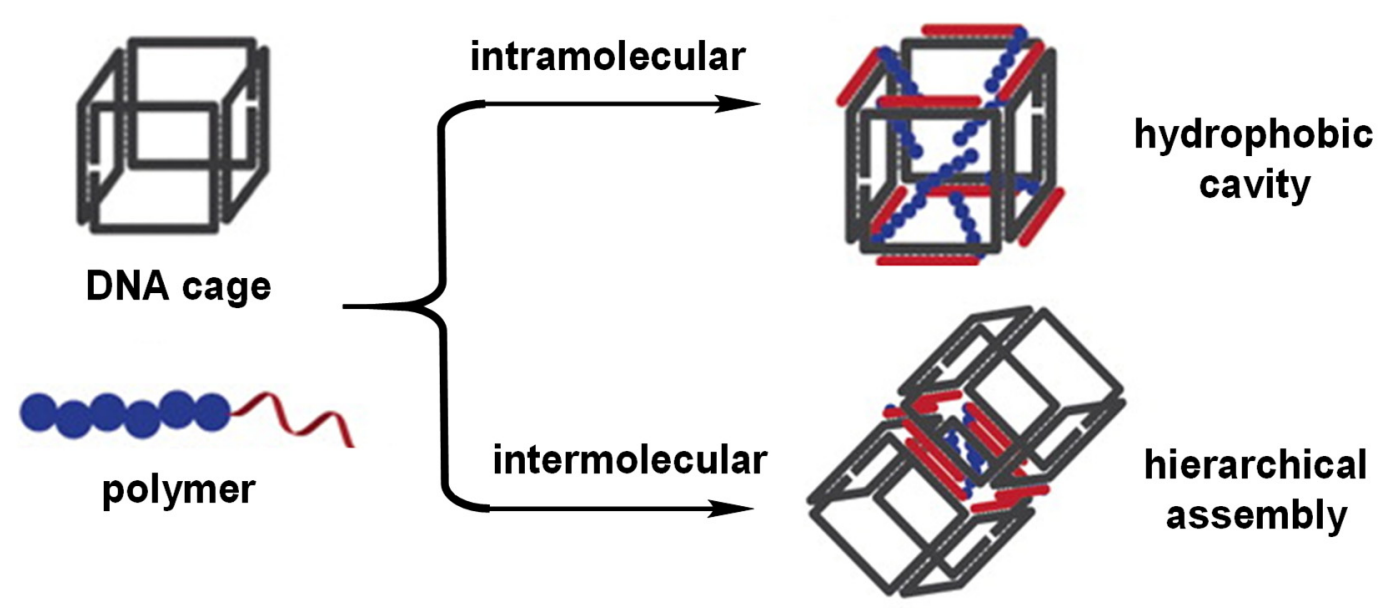

Figure 18. DNA cages functionalised with polymer chains can generate monomeric structures with a hydrophobic core capable of encapsulating hydrophobic guest molecules, or may form hierarchical assemblies through intermolecular polymer chain interactions. The length and polymer sequence, as well as the position of the chain on the cage have all been shown to effect whether intra- or intermolecular interactions dominate. 


\subsubsection{DNA nanopatterning}

Platforms that enable nanoparticles to be arranged according to a systematic design pattern, and to be rearranged after deposition are highly sought after in nanoscience, where such techniques could significantly advance developments in nanocircuitry and photonics. ${ }^{[125,137]}$ By virtue of their well-defined 3D structures, water-solubility and the high sequence selectivity, DNA architectures have been proposed as templates to enable positioning of metal, semi-conductor and magnetic nanoparticles. Initial research by Sleiman and coworkers showed that two-dimensional arrangements of the gold nanoparticles could be programmed through the choice of oligonucleotide linker, and the order in which the oligonucleotides were added to the solution. ${ }^{[113]}$ Gold nanoparticles were imaged by TEM following synthesis of the self-assembling constructs, and after undergoing oligonucleotideprogrammed reorganisation. ${ }^{[137]}$ Changes in the arrangement of metal nanoparticles following introduction of an oligonucleotide trigger demonstrated the write/erase potential of the system. This approach to nanoparticle organisation was developed further in 2015 by Gang and co-workers who reported spatial organisation of gold nanoparticles in three dimensions. Utilising DNA octahedra defined by edges consisting of four six-helix bundles, each containing a single stranded DNA sticky end, Gang and co-workers arranged 7, 10 and $15 \mathrm{~nm}$ gold nanoparticles in a 3D mesoscale arrangement. ${ }^{[138]}$

\subsubsection{Guest binding}

In addition to positioning the nanoparticles on the vertex of DNA nanostructures, there are also reports of nanoparticle encapsulation within DNA architectures. ${ }^{[139]}$ A 2009 publication by Krishnan and co-workers detailed the synthesis of an icosahedral structure, $\mathbf{2 4}$, from two five-fold symmetric half shells (Figure 19A). In the presence of citrate capped gold nanoparticles, the two halves of the icosahedron could be self-assembled and ligated. Following size exclusion chromatography to remove unbound gold nanoparticles, TEM 
indicated clusters of gold nanoparticles comprising of $6 \pm 2$ particles were present. High resolution TEM analysis indicated the gold nanoparticles were encapsulated within the core of 24, occupying approximately $53 \%$ of the computed void space. This fill ratio, defined as the ratio of the guest volume to the host volume, reflects the frequently cited supramolecular ruleof-thumb that states that a host-guest fill ratio of $55 \%$ is optimal. ${ }^{[140]}$ It therefore indicates another area where understanding developed within one discipline, in this case supramolecular chemistry, can be directly transferred to research performed in the adjacent biotechnology sector.

\section{$\underline{\text { 3.1.4 Drug delivery strategies }}$}

Being biologically derived and water-soluble, many studies have evaluated the cellular uptake and stability of DNA architectures. In many cases the DNA constructs tested have demonstrated transport of organic ${ }^{[5,141]}$ and inorganic ${ }^{[114]}$ guests into cells, and have displayed reasonable stability and toxicity ${ }^{[141-142]}$ profiles, essential properties if these constructs are going to be viable drug delivery vehicles.

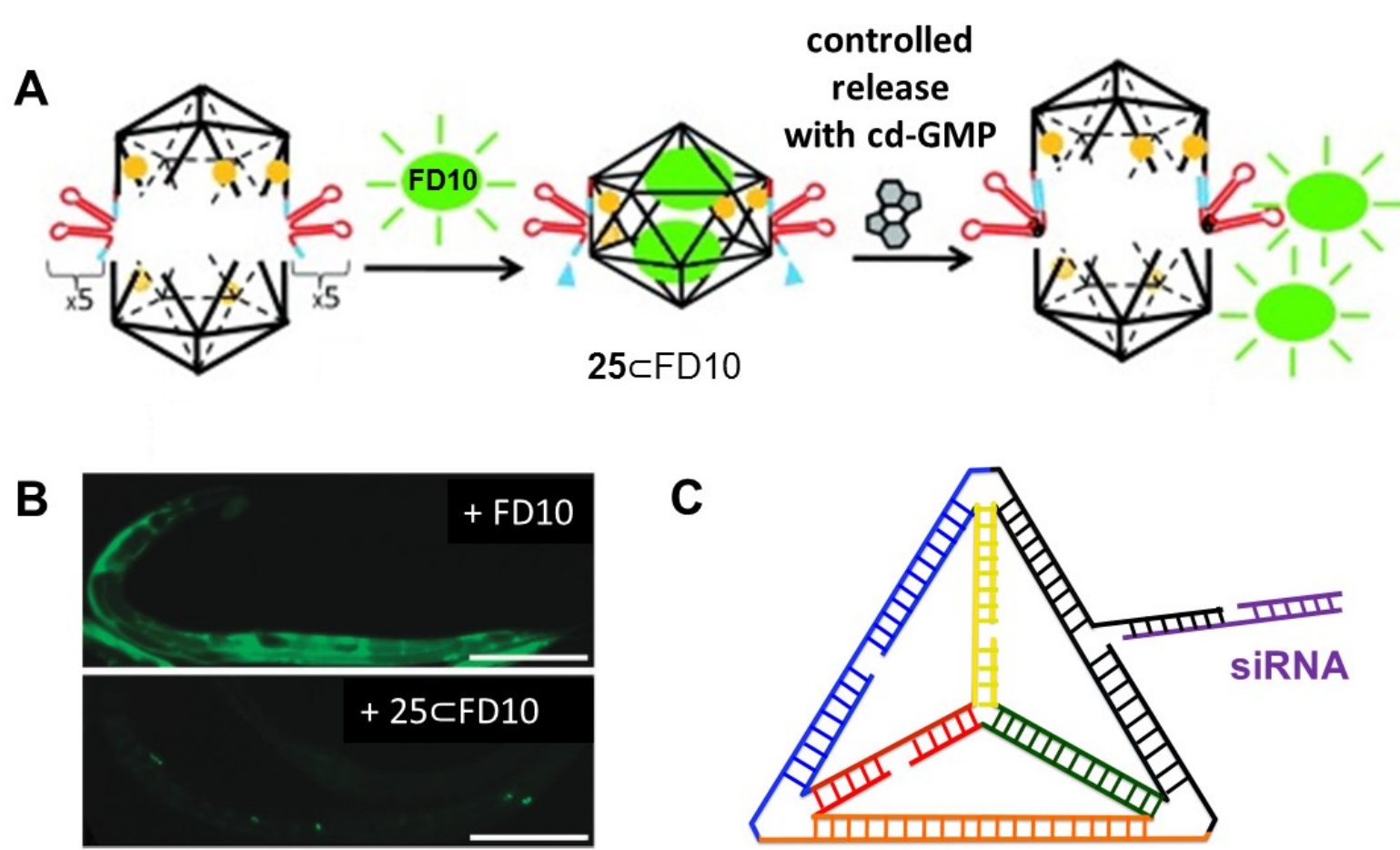

Figure 19. A: Hybridisation of complementary DNA pentamers generates an icosahedral capsule that can, with the aid of an aptamer lock (red) and the cd-GMP key, bind and controllably release guest molecules including the fluorescent dye FD10. B: Encapsulated 
FD10 was differentially taken up in C. elegans; an example of the emergent behaviour that was cited as the driving force for investigating nanostructures. C: Schematic of a DNA tetrahedron with siRNA (purple) attached through complementary hydrogen bonding with a structural DNA strand overhang (black). Release of siRNA is envisioned through disruption of the hydrogen bond interactions.

In a 2011 publication Krishnan and co-workers demonstrated the potential of $\mathbf{2 5}$ for in vivo imaging applications. ${ }^{[5]}$ Exploiting the fluorescence properties of 10kD FITC-dextran (FD10) the authors showed that $\mathbf{2 5} \subset$ FD10 could be taken up into Drosophilia hemocytes. Further experiments probing the mechanism of uptake revealed differential pathways were operative for unencapsulated FD and 25ᄃFD10, and implicated the anionic ligand-binding receptor pathway in 25СFD10 uptake. In vivo experiments in Caenorhabditis elegans supported this observation, with nonspecific distribution of FD10 observed in C. elegans (Figure 19B, upper panel) in contrast to highly localised uptake of 25ᄃFD10 in only the coelomocytes of C. elegans (Figure 19B, lower panel), at equivalent dosages. Coelomocytes are cavities within the C. elegans that take up fluid endocytotically, and thus do not require a receptor to accumulate $25 \subset F D 10$. This observation of differential in vivo processing of a guest upon encapsulation highlights the potential for emergent behaviour within such systems, providing further impetus for development of the field. Receptor-mediated uptake of a biotinfunctionalised octahedral DNA cage has also been reported by Desideri, Biocca and coworkers. ${ }^{[143]}$ This octahedral construct is taken up preferentially by the oxidised low density lipoprotein receptor-1 (LOX-1) which is a tumour and cardiovascular disease marker.

Using a modified version of the icosahedron capsule $\mathbf{2 5}$, controlled release of an encapsulated cargo in response to cyclic di-guanosine monophosphate (cd-GMP) has also been demonstrated. ${ }^{[144]}$ The capsule includes an aptamer at the interface between the two halves of the icosahedron (Figure 19A). In response to cd-GMP the aptamer undergoes remodelling, triggering dissociation of the two half units and release of the encapsulated payload. Controlled release of the payload in response to cd-GMP addition was demonstrated 
using fluorescence assays that looked at quenching of FD10 in response to interactions with external gold nanoparticles, and also loss of Förster resonance energy transfer (FRET) intensity of an Alexa-647-functionalised capsule encapsulating FD10 as a donor fluorophore. A telomerase responsive platinum nanoparticle delivery vehicle has recently been reported, ${ }^{[14]}$ that employs a similar methodological approach to the cd-GMP responsive icosahedron. However, in this case the icosahedron contains telomere primers on the seam between the two half shells, these primers are extended in the presence of telomerase and liberate the encapsulated guest. Since telomerase is expressed in $85-90 \%$ of cancer cells but is almost absent in somatic cells, the telomerase responsive element provides cancer cell selective drug delivery. Cell viability assays and mouse experiments confirm the promising anticancer properties of the encapsulated platinum nanoparticle construct, with the host-guest complex displaying improved efficacy and reduced systematic toxicity compared with either cisplatin or the encapsulated nanoparticles. It should be noted that guest release induced by a biological trigger represents a complementary strategy to the methods discussed in Section 2.4 which covers controlled guest release from synthetic assemblies.

Tetrahedral DNA cages have also been extensively investigated within biological environments. Initial studies demonstrated the cellular uptake and persistence of cages functionalised with the cyanine dyes Cy5 and Cy3 in human embryonic kidney (HEK) cells, and noted that the constructs localised mostly to the cytoplasm. ${ }^{[142]}$ These results paved the way for subsequent reports of DNA tetrahedra functionalised with CpG nucleotides. ${ }^{[145]}$ Therapeutic $\mathrm{CpG}$ nucleotides are challenging to deliver into cells, even with the aid of transfection agents, and are readily degraded once inside the cell. When appended to the tetrahedron, however, $\mathrm{CpG}$ nucleotides were shown to be stable for up to four hours in fetal bovine serum (FBS), in contrast to the free $\mathrm{CpG}$ nucleotide that was completely deactivated within two hours under the same conditions. Utilising a similar approach Anderson and co- 
workers appended short interfering RNA oligonucleotides (siRNA) and targeting moieties to DNA tetrahedra (Figure 19C). ${ }^{[141]}$ DNA tetrahedra functionalised with at least three folate ligands demonstrated robust gene silencing in mouse models, indicating good cellular uptake of the siRNA. Furthermore, no immune response was reported in response to administration of the siRNA-functionalised tetrahedra.

Sleiman and co-workers have also reported conditional release of siRNA from a 'nanosuitcase', in response to a genetic marker. ${ }^{[146]}$ The DNA cuboid reported in this case binds the siRNA between two diagonally opposite edges such that the siRNA is completely surrounded by the framework of the DNA host. The DNA scaffolding is thus able to protect the encapsulated siRNA, increasing its half-life four-fold over the non-encapsulated siRNA in simulated biological conditions. Release of the encapsulated siRNA strand was triggered by complementary base pairing of messenger RNA with DNA strands that are partially involved in siRNA binding, thus enabling stimuli responsive displacement of the siRNA. Once released the siRNA could affect gene expression in cells, and thus this construct has potential as a method of controlled drug delivery.

\section{$\underline{3.1 .5}$ Protein binding}

In contrast to synthetic cages where encapsulation of small, hydrophobic molecules has been the focus (see section 2.3), the increased size and polarity of the cavity found in DNA cages makes them better suited for protein encapsulation. Numerous examples have been reported of protein encapsulation within DNA cages, and in some circumstances controllable release of the protein in response to a biomolecule, or change in temperature have been reported.

In 2006, Turberfield and co-workers reported encapsulation of cytochrome c within a DNA tetrahedral cage (Figure 20A) following conjugation of cytochrome c with the $5^{\prime}$ end of one of the constituent DNA strands. ${ }^{[21]}$ Varying the nucleotide the protein was attached to, 
altered the binding site of cytochrome $\mathrm{c}$ on the DNA strand and enabled the researchers to direct the protein to be bound inside or on the exterior of the tetrahedron. Subsequent reports have utilised non-covalent binding to direct protein encapsulation. As a proof of concept, a tetrahedron containing a 22 base pair consensus binding sequence for the transcription factor catabolite activator protein (CAP) has been shown to bind CAP with high affinity in the presence of cyclic adenosine monophosphate. ${ }^{[26]}$ The authors postulate that CAP binding within the cavity of the cage requires considerable conformational changes in the structure of the tetrahedron as well as CAP. Once bound inside the cage, CAP was shown to dissociate much more slowly from the binding sequence than it did in the absence of the tetrahedral framework, as might be expected. Controlled release of CAP from the cage upon disassembly with DNA nuclease I was also shown to be possible.

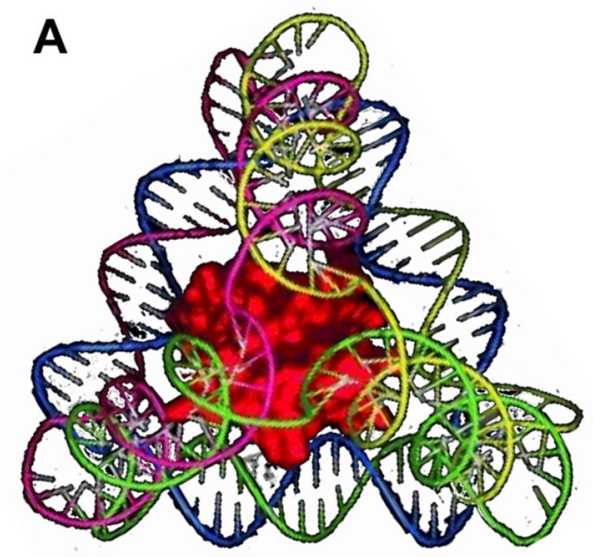

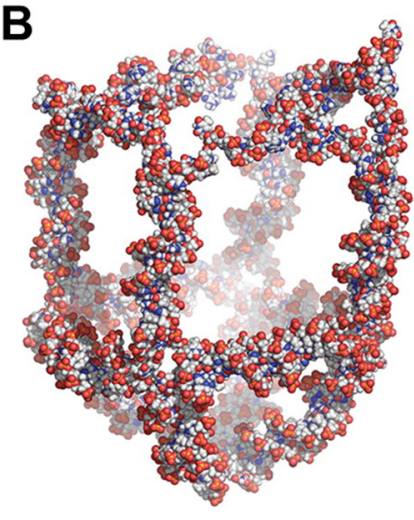

26

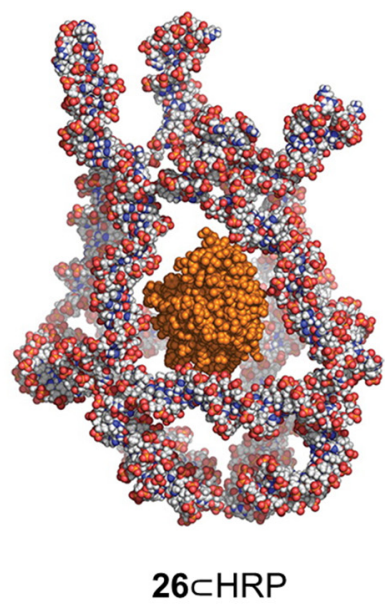

Figure 20. A: Model of encapsulated cytochrome c (red) bound within a DNA tetrahedron. B: model of cage 26 in its open form (centre), and following cooling to $4{ }^{\circ} \mathrm{C}$ in the presence of a guest (HRP) when it was found to be in its closed form (right).

The host-guest binding of the model antigen streptavidin inside a $\mathrm{CpG}$-functionalised tetrahedral cage, and its cellular uptake and processing has also been reported. ${ }^{[147]}$ The DNA scaffold was shown to promote uptake of both the streptavidin and the CpG nucleotides relative to their unbound forms. Once inside the cells the antigen-CpG-DNA construct was 
shown to promote a strong, long-lasting antibody response, highlighting the potential of these highly modular DNA constructs as synthetic, multivalent vaccine complexes.

Horseradish peroxidase (HRP) has also been encapsulated in a truncated octahedral DNA cage containing a hairpin-forming DNA sequence (26; Figure 20B) that enabled temperature controlled encapsulation and release of the enzyme. ${ }^{[148]}$ At $4{ }^{\circ} \mathrm{C}$ annealing of the hairpin sequence generated the closed cage structure, and inhibited encapsulation of the enzyme. After heating to $37^{\circ} \mathrm{C}$ the hairpin unfolded enabling encapsulation of HRP, and upon subsequent cooling to $4{ }^{\circ} \mathrm{C}$ the HRP remained bound inside 26 now in its closed conformation. HRP remained active when bound in closed 26, and could be controllably released upon heating to $37^{\circ} \mathrm{C}$ when $\mathbf{2 6}$ was returned again to its open form.

Bringing many of the aforementioned functions together Church and co-workers reported the design and synthesis of an aptamer-gated hexagonal DNA barrel capable of interfacing with cells to deliver preprogrammed responses, in the form of cell signalling molecules. ${ }^{[117]}$ The DNA aptamer forming the lock could be opened in response to either one or two antigen signals depending on the design of the "robot", while the choice of payload included $5 \mathrm{~nm}$ gold nanoparticles or a range of (antigen-binding fragment) Fab' antibodies. Cell studies confirmed that in the presence of the correct "key" combination the barrel was unlocked and the antibodies were released. Once released the antibodies were then either quantified by fluorescence measurements, or prompted a cellular response depending on the experimental design. Inclusion of antibodies to human CD33 and CDw328 Fab' fragments generated robots that induced growth arrest in NKL cells derived from a patient with granular lymphocytic leukaemia.

Recently, peptide nucleic acids (PNA) have been proposed to extend the field of DNA self-assembly and functionality. ${ }^{[149]}$ This approach utilises complementary interactions between nucleobases on a preassembled DNA nanocage to direct self-assembly of PNA 
peptides in the DNA cage void, thus providing a rational approach to direct polypeptide engineering. This methodology also provides an interesting approach to synthetic generation of protein active sites from several short polypeptides appropriately orientated and positioned by the DNA framework.

\subsection{Peptide-based architectures}

\subsubsection{Design of peptide architectures}

Understanding how the primary amino acid sequence of a protein influences its tertiary structure has made it possible to predict the configuration of these folded assemblies. This sequence-to-structure relationship allows prediction of secondary structural motifs, and subsequently the 3D structure of the protein, ${ }^{[150]}$ whilst de novo design and prediction has been made possible with computational programmes such as CCBuilder and ROSETTA. ${ }^{[151-}$ 154]

Classical coiled-coil de novo peptides utilise favourable interactions between secondary $\alpha$-helical units. These motifs are based on a seven-residue repeat comprised of hydrophobic $(h)$ and polar $(p)$ amino acids, usually denoted hpphppp or abcdefg and represented by helical wheel diagrams (Figure 21). ${ }^{[155]}$ The oligomerisation state, the number and orientation of the individual $\alpha$-helical units within a coiled-coil structure, is dictated by the position and identity of the constituent amino acid residues. When the helices coil, the $a$ and $d$ positions are brought together to form a hydrophobic face. ${ }^{[156-157]}$ These residues also determine whether the helices are structured in a parallel (Figure 21A) or anti-parallel (Figure 21B) orientation with respect to each other. It is known that positioning isoleucine (Ile) residues in the $a$ position and leucine (Leu) in the $d$ position promotes the formation of parallel dimers. ${ }^{[157]}$ Introduction of Ile in the $d$ position will promote the formation of trimers 
(Figure 21C), unless $a$ is a Leu residue in which case the formation of a tetrameric structure is favoured (Figure 21D). ${ }^{[157]}$

The stability and oligomerisation state of coiled-coils can also be influenced by interhelical ionic pairs that form between charged residues in the $e$ and $g$ positions. ${ }^{[158-159]}$ Inclusion of glutamic acid (Glu) and lysine (Lys) frequently promote the association of coiled-coils, usually specifying a trimeric oligomerisation. ${ }^{[157,159]}$ Glutamine (Gln) in both the $e$ and $g$ position supports dimer formation as no interhelical electrostatic interactions disrupt the structure. ${ }^{[159]}$

A
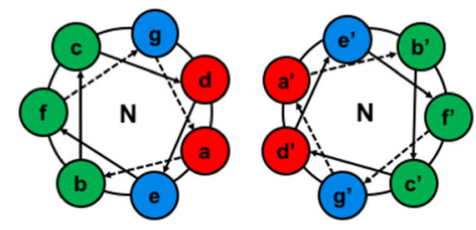

C

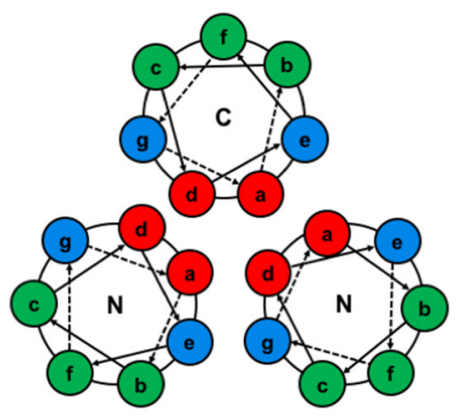

B

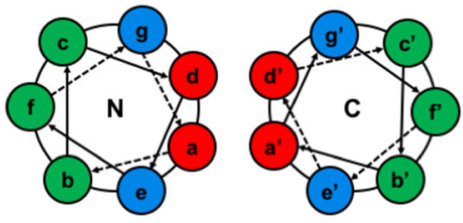

D

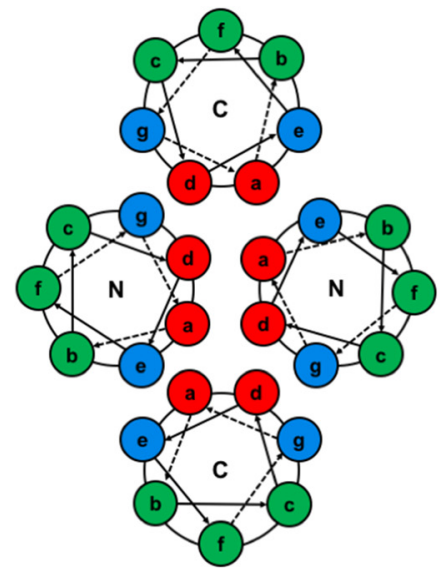

Figure 21. Helical wheel diagrams showing; A: parallel dimeric coiled-coil; B: anti-parallel coiled-coil; C: trimeric coiled-coil; D: tetrameric coiled-coil. N and C identify the N- and Cterminus of the peptide sequence, respectively.

The likelihood and strength of cooperative, non-covalent interactions between residues on adjacent helices of coiled coils have also been assessed with a view to guiding future design efforts. For a system containing three or more different coiled-coil forming peptides, thermodynamically driven selective dimer formation can occur, revealing the highest-affinity interactions. ${ }^{[160]}$ Aili and co-workers validated this concept by mixing four de novo peptides, 
$\mathbf{E I}, \mathbf{E V}, \mathbf{K I}$ and $\mathbf{K V}$, that self-assemble into four different coiled-coils. The peptides have variable affinities for each other with $K_{\mathrm{d}}$ values ranging from $10^{-6}$ to $<10^{-10} \mathrm{M}$. The strongest interaction was found between $\mathbf{E I}$ and $\mathbf{K I}$ due to a short asparagine $\cdots$ asparagine (Asn) hydrogen bond (Figure 22A). Evidence for selective dimer formation in the system was supported by fluorescence measurements performed with a modified EV peptide that had a Cy5 fluorophore grafted at the N-terminus (EV-Cy5). ${ }^{[160]}$ Upon addition of a complementary peptide, KI, an increase in the $\mathrm{Cy} 5$ fluorescence emission intensity was reported, consistent with coiled-coil formation. A corresponding decrease in fluorescence intensity was reported upon subsequent addition of EI, which displaced $\mathbf{E V}-\mathbf{C y} \mathbf{5}$ from the duplex, generating $\mathbf{E I} \cdot \mathbf{K I}$ as the most stable adduct alongside free EV-Cy5 (Figure 22B). ${ }^{[160]}$
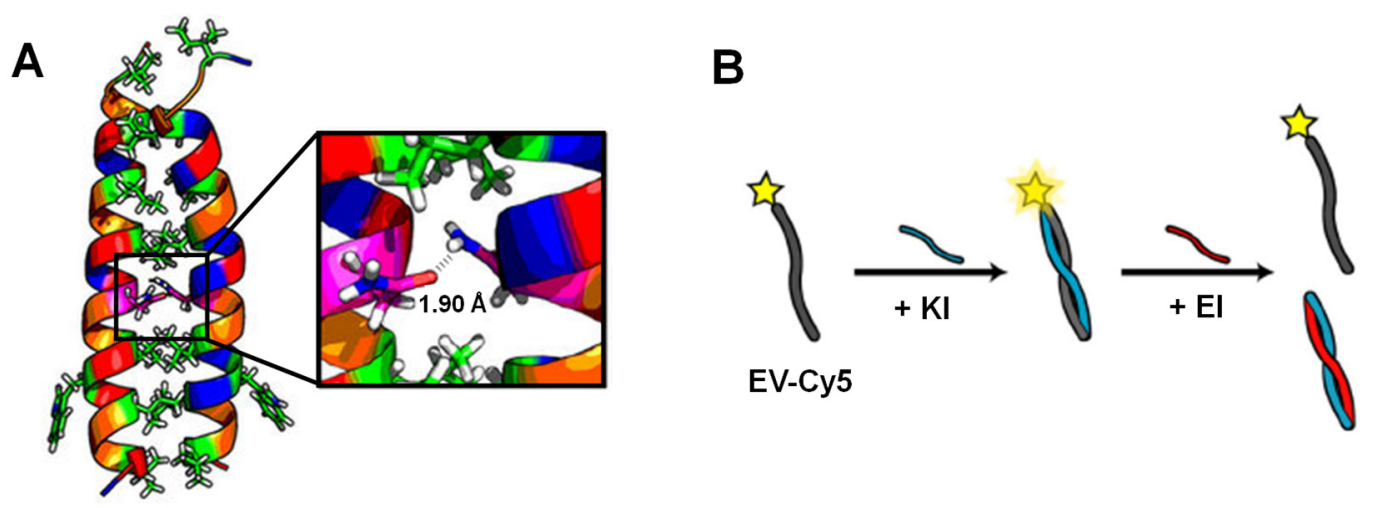

Figure 22. A: The short Asn $\cdots$ Asn hydrogen bond present in peptide EI·KI and; B: the displacement of EV-Cy5 by $\mathbf{E I}$ as a result of the strong interaction between Asn residues in EI $\cdot \mathbf{K I}$

De novo design has also enabled rational construction of metal coordination sites between $\alpha$-helices; metals coordinated in these sites may perform a structural or functional role. Modification of the $a$ and $d$ residues, has been shown to generate a metal ion binding site within the hydrophobic core of the helix bundle, that destabilises the assembled conformation in water, forcing the peptide to exist as a random structure. ${ }^{[161]}$ However, upon addition of a metal ion with the appropriate coordination geometry, it is possible to induce the selfassembly of the desired coiled-coil through favourable interactions. ${ }^{[161]}$ Gaining structural 
control of this self-assembly process has enabled the first applications of peptides architectures, as artificial catalysts ${ }^{[162]}$ and MRI contrast agents, ${ }^{[163]}$ to be realised.

Tanaka and co-workers first demonstrated that the introduction of two hydrophilic histidine (His) residues within the hydrophobic core of a known triple-stranded coiled-coil could simultaneously destabilise the folded structure whilst creating a potential site for metal ions to bind. Both His residues in each of the three helices in this structure can bind a metal ion in a monodentate fashion, creating a six-coordinate site that binds $\mathrm{Ni}$ (II), $\mathrm{Co}$ (II) and $\mathrm{Zn}$ (II) in an octahedral manner. ${ }^{[161]}$ Circular dichroism was used to confirm that in the absence of metal ions, the peptide exists as a random coil in an aqueous solution, whilst addition of an appropriate metal induced the assembly of a parallel, triple-stranded coiled-coil. ${ }^{[161]}$ Subsequent research indicated that replacing the two His residues, with an alanine (Ala) residue in the $d$ position and a cysteine (Cys) residue in the $a$ position of the peptide, promoted binding of softer metals in a low-coordinate, trigonal fashion. ${ }^{[164]}$ Circular dichroism experiments confirmed that higher-coordinate $\mathrm{Co}(\mathrm{II}), \mathrm{Ni}(\mathrm{II})$ and $\mathrm{Zn}(\mathrm{II})$ did not induce folding of the modified peptide, whilst softer, lower-coordinate $\mathrm{Cd}(\mathrm{II})$ and $\mathrm{Hg}$ (II) did, exemplifying how small sequence alterations can significantly affect the metal ion binding affinity and related 3D structure of the peptide.

The combination of His and Cys binding motifs had previously been explored by Regan and Clarke, who engineered a tetrahedral coordination site within the hydrophobic core of a four-helix bundle protein. ${ }^{[165]}$ The modified protein consisted of four helices linked by three loops, and the metal binding site was created between two adjacent helices. Coordination of tetrahedral $\mathrm{Zn}$ (II) and $\mathrm{Co}(\mathrm{II})$ ions was confirmed using a variety of spectroscopic and chromatographic techniques. It was also concluded that, compared with the apoprotein, the $\mathrm{Zn}(\mathrm{II})$ bound protein was significantly stabilised against denaturation by guanidinium chloride. ${ }^{[165]}$ 
More recently Pecoraro and co-workers reported an in-depth study into tristhiolate binding sites, concluding that rotation and reorganisation of the three Cys residues in the metal binding site, depend exclusively on the preferred geometry of the metal ion and is independent of metal ion size or charge. ${ }^{[166]}$ A similar dependence on coordination geometry is observed in the formation of synthetic metal-organic constructs, where changes in the charge $^{[167]}$ and sometimes size ${ }^{[168]}$ of the metal ion do not alter the structure. Pecoraro and coworkers also determined that the tristhiolate binding site is prearranged to bind metals in a trigonal pyramidal fashion, but is inclined to reorganise upon introduction of metals that favour trigonal planar geometries. ${ }^{[166]}$

It has also been demonstrated that metal binding sites are not restricted to the hydrophobic core of coiled-coil assemblies. In 1998 Hodges and co-workers demonstrated the binding of high-coordinate $\mathrm{La}(\mathrm{III})$ ions through destabilising $\gamma$-carboxyglutamic acid residues in the outer $e$ and $g$ positions of a two-stranded coiled-coil. ${ }^{[169]}$ The ionic repulsion between the adjacent carboxylate groups forced unfolding of the peptide in aqueous solution in the absence of a metal ion. Subsequent introduction of $\mathrm{La}(\mathrm{III})$ and $\mathrm{Yb}(\mathrm{III})$ ions allowed the peptide to dimerise and the interstrand disulfide bridge directed the formation of a parallel coiled-coil complex.

Disulfide bridges have more recently been utilised in organic chemistry to drive the formation of synthetic cages in aqueous solution. ${ }^{[170]}$ Building on extensive research into the generation of disulfide-linked catenanes ${ }^{[171-172]}$ Sanders and Stefankiewicz reported a series of disulfide-linked cage molecules in 2012. ${ }^{[173]}$ These cages self-assembled from a combination of di- and trithiol linkers in the presence of a positively charged amine guest.

With growing understanding of sequence-to-structure relationships and thus coiledcoil interactions, designing and predicting the structure of 2D peptide architectures de novo has become an attractive area of research. Keating and co-workers reported the de novo 
design and formation of a 2D protein nanotriangle, consisting of three thermally annealed chains of linked coiled-coils (Figure 23A). ${ }^{[174]}$ The linker was a flexible (Gly 4 Ser) 2 protein segment of fixed length, and, unlike previously synthesised protein triangles, the peptide construct assembled in the absence of metal ions. ${ }^{[175]}$ The effect of linker length had previously been examined using a (GlyAsn) $x$ linker, where $x$ was varied from one to five. ${ }^{[176]}$ Discrete, closed structures were postulated to form for $x=3-5$ following sample annealing, with analytical centrifugation experiments and computational models supporting formation of tetramers $(x=3)$, trimers $(x=4)$ and a mixture of trimers and dimers for $x=5$ (Figure 23B). ${ }^{[176]}$

A
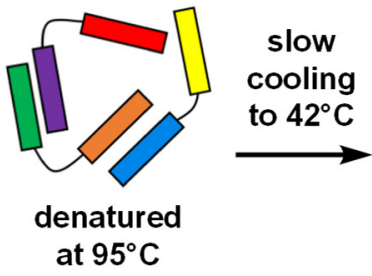
at $95^{\circ} \mathrm{C}$

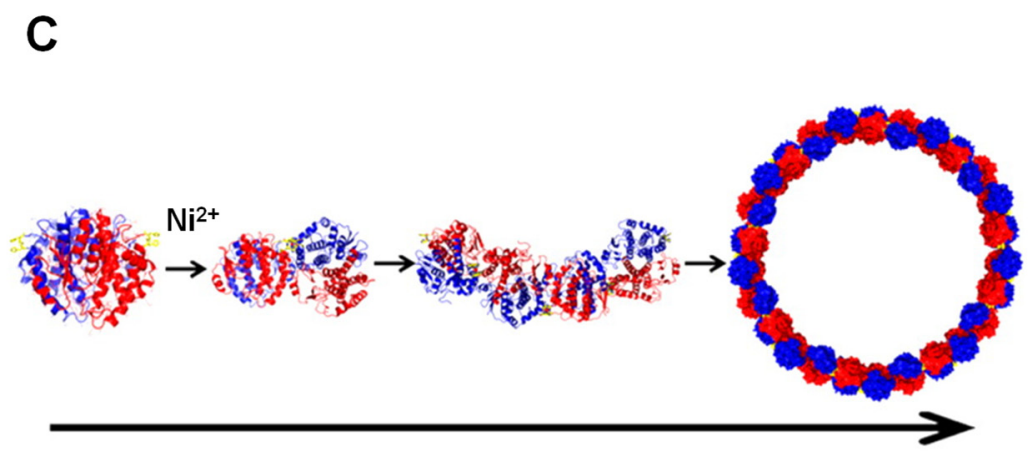

B

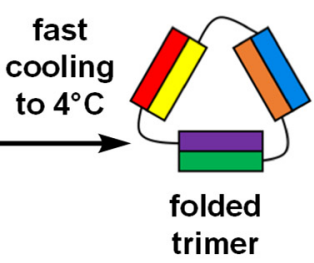

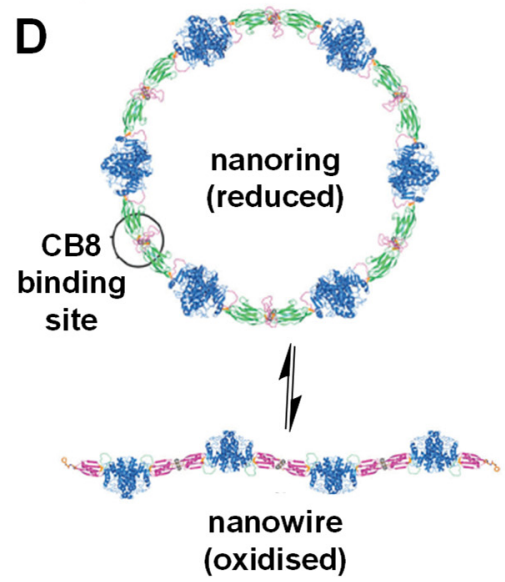

Figure 23. A: Thermal annealing of a protein nanotriangle. B: Dimeric, trimeric and tetrameric structures postulated to form upon decreasing the peptide linker length (from $x=$ 5-3) between two heptad repeat sequences shown in red and blue. C: Stepwise growth of a peptide nanoring through the self-assembly of peptide building blocks: upon addition of nickel the bis-histidine binding sites (indicated in yellow) of adjacent peptide units coordinate in a pairwise manor generating an extended structure which due to its curvature closes as a circle. D: The two architectures observed when glutathione S-transferase was in its i) oxidised state (pink peptide) a nanowire and; ii) reduced state (green peptide) a nanoring. 
Self-assembly of 2D peptide nanostructures was also shown to be mediated through coordination of metal ions. Introduction of a bis-His metal binding site into a homodimeric coiled-coil was achieved by Liu and co-workers, creating a peptide construct that bound $\mathrm{Ni}(\mathrm{II}) \cdot{ }^{[177]}$ Metal coordination triggered the self-assembly and growth of a protein nanoring (Figure 23C). More recently, self-assembly of a protein nanoring was achieved under reducing conditions in the presence of $\mathbf{C B 8}$. CB8 in this instance formed a 1:2 complex with a terminal Phe-Gly-Gly peptide sequence thus linking adjacent building blocks. Inclusion of redox active glutathione S-transferase, that changed conformation with changes in oxidation state (Figure 23D, green peptide reduced, pink peptide oxidised) within the building block, resulted in structural conversion between a nanoring and an extended nanowire upon addition of oxidant. ${ }^{[178]}$

In addition to 2D self-assembled structures, discrete 3D constructs can also be formed from combinations of de novo peptides. Hubs generated by disulfide bond formation between a coiled-coil homotrimer, $\mathbf{C C}$-Tri, and acid and base functionalised coiled-coils (CCDi-A and CCDi-B, respectively) were observed to spontaneously self-assemble in water to generate large (diameter: $79 \pm 12 \mathrm{~nm}$ ) spherical cage-like particles (Figure 24A). ${ }^{[179]}$ Formation of this assembly was underpinned by the thermodynamically favourable coiled-coil interactions, with association of complementary CCDi-A and CCDi-B termini providing the driving force for self-assembly of complementary charged hubs. ${ }^{[179]}$ Three-dimensional structures have also been developed through the combination of cyclic peptides and heterobifunctional spacers, ${ }^{[180]}$ and can be manipulated for small-molecule recognition. ${ }^{[181]}$ 
A

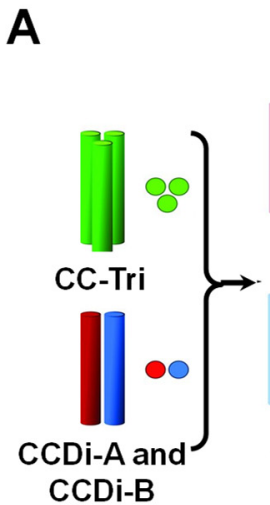

C

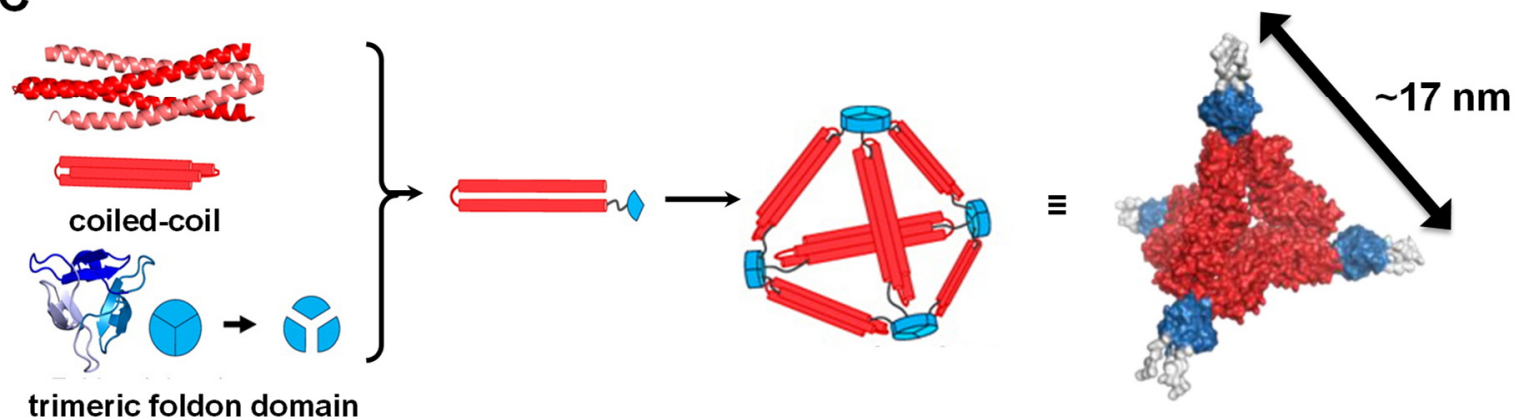

B

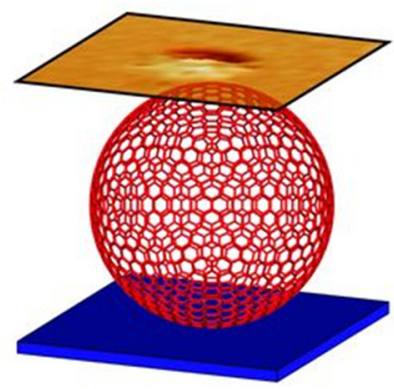
lonic interactions

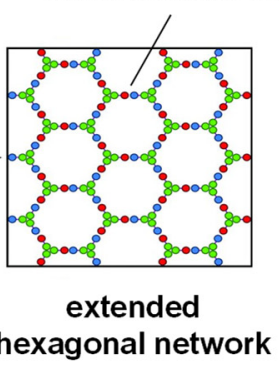

hexagonal network
Coiled-coil presenting

peripheral base groups

trimeric foldon domain

Figure 24. Representation of structures formed through the self-assembly of peptide subunits. A: Trimeric coiled coil CC-Tri (green) and CCDi-A (red) and CCDi-B (blue) subunits brought together to generate an extended hexagonal network that curves to generated B: a spherical assembly. C: A schematic representation of the tetrahedron generated from coiledcoils (red) and the trimeric foldon domain of T4 fibritin (blue); and a 3D model of the tetrahedron-shaped dodecamer recreated using SAXS data.

Several different methods have been reported for the assembly of peptides into tetrahedral architectures. In 2013, Jerala and co-workers reported formation of a peptide tetrahedron from a single polypeptide chain containing twelve coiled-coil forming sections. ${ }^{[182]}$ An alternative approach involving the combination of peptide building blocks with different symmetries was reported in 2015 by Arai and co-workers. ${ }^{[183]}$ This methodology utilised a fused de novo dimeric coiled-coil that folds to form a 4-helix peptide bundle, with the trimeric foldon domain of bacteriophage T4 fibritin. This design was used to generate a range of nanoarchitectures, including a tetrahedron-shaped dodecamer (Figure 24B). ${ }^{[183]}$ A tetrahedron was also reported in 2017 by Marsh and co-workers, who combined a $C_{3}$-symmetric trimeric 
coiled-coil and a $C_{3}$ trimeric protein with an eight-residue Gly linker to form the tetrahedral construct. $^{[184]}$ The same trimeric protein had previously been coupled with a tetrameric coiledcoil to form an octahedral assembly, and with a pentameric coiled-coil to form an icosahedron. ${ }^{[184]}$

A large cubic structure has also been reported to form from the combination of dimeric and trimeric proteins connected through an $\alpha$-helical linker. The self-assembled structure has a cavity $130 \AA$ in diameter ${ }^{[28]}$ and is much larger than cages previously formed using protein oligomers, including the tetrahedral assembly generated in an analogous fashion from the fusion of trimeric and dimeric units, that was reported to enclose a void space measuring approximately $50 \AA$ in diameter. ${ }^{[185]}$

\subsubsection{MRI contrast agents}

The biological basis and relative ease of manipulation associated with coiled-coil constructs makes them attractive for medicinal use. In particular, combining the biomolecular recognition potential of coiled-coil assemblies with the reported efficacy of paramagnetic metals has resulted in the development of promising contrast agents for MRI imaging. ${ }^{[186]}$ Introduction of a potential lanthanide binding site within the hydrophobic core of a threestranded coiled-coil bundle resulted in a complex with superior relaxivity to the commercial agent DOTAREM ${ }^{\circledR} \cdot{ }^{[163]}$ Unexpectedly, detailed analysis of the complex concluded there were no water molecules coordinated to the metal ion, a feature commonly associated with effective contrast agents.

Further investigation established that the position of the binding site strongly correlated with the relaxivity of the complex. When the metal was bound closer to the terminus, rather than the centre, of the three-helix bundle an increase in peptide stability was reported, despite the increased number of coordinated water molecules. ${ }^{[163,187]}$ This dependence on location was rationalised by the feasibility of the bundle to adapt to 
conformational changes in its structure, enabling coordination of water molecules and imparting the desirable relaxivity properties.

\subsubsection{Enzyme mimics}

As well as biomedical functionality, it has also been possible to design constructs from coiledcoils that can mimic naturally occurring catalytic metalloproteins. Using the coiled-coil ligands to support catalytically active metal sites is a complementary approach to development of novel catalysts from synthetic 3D structures than that presented in section 2.2. Conceptually, development of constructs that utilise the hydrophobic effect in combination with a catalytic metal site have the potential to show significant improvements in selectivity and rate over mimics incorporating either one of these features alone.

Human carbonic anhydrase II (CAII) catalyses the hydration of $\mathrm{CO}_{2}$ at a zinc metal active site within the enzyme. Based on a three-stranded coiled-coil bundle, Pecoraro and coworkers designed and synthesised a de novo metalloenzyme with two unique metal binding sites capable of accommodating the structural metal ion, $\mathrm{Hg}(\mathrm{II})$, and a catalytic $\mathrm{Zn}$ (II) metal ion. This construct was reported to catalyse the hydration of $\mathrm{CO}_{2}$ and hydrolysis of $p$ nitrophenyl at remarkable rates. ${ }^{[162]}$ Although not as efficient as native human CAII for $\mathrm{CO}_{2}$ hydration, this designed metalloprotein was shown to be 70 -fold faster than previously reported CAII mimics. Based on the same three-stranded peptide bundle used for the CAII mimic, a $\mathrm{Cu}(\mathrm{I}) / \mathrm{Cu}(\mathrm{II})$ analogue was produced. In aqueous solution, this metalloprotein was reduced using ascorbate, and the resultant $\mathrm{Cu}(\mathrm{I})$ active site shown to act as a reducing agent, catalysing the reduction of $\mathrm{NO}_{2}$ to $\mathrm{NO} \cdot{ }^{[188]}$

The requisite symmetry of parallel three-strand metallo-bundles make it difficult to make alterations that will systematically improve catalytic activity as modifications must be accommodated in all three strands. Pecoraro and co-workers thus designed and synthesised a single-stranded antiparallel coiled-coil assembly capable of catalysing the hydration of $\mathrm{CO}_{2}$ at 
a $\mathrm{Zn}$ (II) metal centre albeit at slow hydration rates. ${ }^{[189]}$ The major advantage presented by the single-strand construct is the ease of non-symmetric residue modification which is limited in classical coiled coil systems.

Modification of the single-stranded bundle with Cys residues has also resulted in the generation of softer metal binding sites capable of binding metals such as $\mathrm{Hg}(\mathrm{II}), \mathrm{Cd}(\mathrm{II})$ and $\mathrm{Pb}(\mathrm{II})$, paving the way for the development of heavy metal sensors. ${ }^{[190]}$

In aqueous solution, copper derivatives of Cys rich metallothioneins have also been reported which display luminescence attributed to the $\mathrm{Cu}(\mathrm{I})$-sulfur chromophore at $600 \mathrm{~nm}$. EXAFS data indicates a trigonally coordinated $\mathrm{Cu}-\mathrm{S}_{3}$ cluster exists within the metal binding site of metallothionein, where up to $12 \mathrm{Cu}(\mathrm{I})$ ions can interact with 20 thiolate groups in the protein. ${ }^{[191-192]}$ Oxidative quenching data revealed the $\mathrm{Cu}(\mathrm{I})$ cluster is an active redox centre for electron transfer, and introduction of such site into a stable, well-understood coiled-coil motif should be possible. A Cys binding site was therefore engineered into a known coiledcoil sequence, and following the addition of $\mathrm{Cu}(\mathrm{I})$ resulted in the formation of a trigonal $\mathrm{Cu}_{4} \mathrm{~S}_{4}(\mathrm{~N} / \mathrm{O})_{4}$ cluster bound within the core of a 4 -stranded coiled-coil. ${ }^{[193]}$ This complex displays significant luminescence and when photoexcited undergoes electron transfer to a number of ruthenium ammine acceptors, providing an interesting foundation for synthetic electron transfer proteins.

Taking inspiration from natural protein structures, supramolecular chemists have developed synthetic molecules known as foldamers that adopt folded conformations similar to those found in peptides. Recently the encapsulation of polar guest molecules in foldamers has been achieved in aqueous solution with an oligourea hexamer demonstrating encapsulation of primary alcohols, ${ }^{[194]}$ and with an indolocarbazole-based foldamer binding $\mathrm{F}^{-}, \mathrm{Cl}^{-}$and $\mathrm{Br}^{-}$in water ${ }^{[195]}$ Further development of these bio-inspired synthetic constructs to accommodate 
metal binding sites, analogous to those incorporated within the coiled coils discussed above, may be a viable route to expanding the scope of foldamers.

\section{Summary and Outlook}

This review summarises the recent progress that has been made across the broad field of discrete, water-soluble self-assembly and the exciting applications that are beginning to emerge that are unique to these three-dimensional constructs. For bio-derived structures the challenges associated with: i) rational development of discrete 3D architectures utilising biological components, attributed to the lack of rigidity in these natural polymers; and ii) the absence of a simple methodology for generation of well-defined vectors, have been tackled. In parallel, developments in the design of synthetic architectures have led to an improved understanding of the factors that give rise to water-soluble synthetic structures. Viewed holistically it is clear that the increased rigidity brought about by the polyaromatic ligands typically used in their supramolecular synthesis is at the expense of water solubility. For DNA and peptide structures, rigidifying the intrinsically water-soluble polymeric chains used to bridge between vertices is achieved by duplex DNA and alpha coil formation, respectively, and ensures that a favourable balance is met between pre-organisation and flexibility.

With these discipline-specific synthetic hurdles cleared, the fields of synthetic and bioderived aqueous self-assembly have reached a point where synthesis of the desired constructs is no longer a limiting factor, allowing more focus on potential applications, as outlined in this review. As previously noted, the supramolecular and nanobiotechnology communities have traditionally been seen as distinct despite sharing common goals - both communities seek to exploit the advantages of compartmentalisation that are observed in natural systems and utilise common design methodologies. ${ }^{[24-25]}$ Going forward we propose that merging these 
two well-developed fields of study will enable more rapid progress in the development of real-world applications from discrete, aqueous assemblies.

A more concerted research effort bridging the two previously distinct areas of research will enable researchers to share research advances, while also ensuring that fundamental differences between synthetic and bio-derived architectures are optimally utilised. Taken together it is apparent that the scale of the architectures created by the two communities tends to differ, with much larger architectures being generally reported for biologically derived constructs, typically in the nanometer size regime, ${ }^{[28]}$ versus the Ångström-sized architectures reported commonly for synthetic architectures. ${ }^{[29-30]}$ Careful consideration of the currently available building blocks therefore allows interaction with both small species, like ions, at one end of the spectrum and with macromolecular entities, such as proteins, at the other end of the spectrum, thus enabling control across the entire size regime typically utilised to modulate biological processes. ${ }^{[196-197]}$ Similarly, utilising constructs that are inherently biodegradable may be an advantage or a disadvantage, depending on the targeted application. Materials derived from DNA or peptide sequences may be leveraged for applications where long-term retention or accumulation of synthetic materials is undesirable, such as drug delivery. ${ }^{[198]}$ An additional benefit of utilising biologically derived components is the ability to reengineer known biological features within new constructs. An excellent early example was the inclusion of a DNA binding sequence as one of the edges of a tetrahedral DNA capsule, enabling non-covalent interactions with a protein to be programmed into the structural elements. ${ }^{[26]}$ Conversely, identifying substrates that will spontaneously bind with aromatic ligands is noticeably harder, however some success in creating architectures with protein size dimensions that can be recognised by specific biological elements has been reported. ${ }^{[102,199]}$ The development of more hybrid, water-soluble constructs ${ }^{[200]}$ that can combine beneficial features from both synthetic and biological derived components is another 
interesting approach that will open the door to discovery of new, emergent properties that are unattainable in the individual research fields. The development of DNA cages with photoresponsive elements or metal-organic capsules with an internal recognition site for biomolecule binding or sensing present two interesting possibilities. Numerous other examples undoubtedly exist.

In addition to continuing synthetic efforts, more detailed characterisation and analysis is also required if researchers are to fully understand the origins of the unique behaviour demonstrated by these architectures in water. In this regard, many excellent catalysts have been reported that utilise hydrophobic binding pockets. Consideration of the long-term catalyst stability, turnover number, catalyst recovery and scalability -parameters typically not evaluated by supramolecular chemists- need to be assessed if the promise of industrially viable catalytic transformations in water mediated by $3 \mathrm{D}$ cages is to be met. Furthermore, for intrinsically water-soluble constructs the potential of these hollow assemblies in transportation and delivery of bioactive guests should be rigorously explored in vivo, and, if possible, a set of structure-activity relationships defined that provide a framework to guide future studies with these unique materials.

More detailed physical studies are also required to better understand the role of water molecules within nanometer-sized frameworks. The recent report of "high-energy" water, ${ }^{[74]}$ which describes water molecules with non-saturated hydrogen bonding arrangements, begins to acknowledge that water molecules isolated inside a cavity should not be considered analogous to water molecules in the bulk phase. Indeed within biological systems the role of individual water molecules that play explicit roles within chemical transformations, has long been known. ${ }^{[201]}$ Similarly, the behaviour of water at interfaces, is known to differ from that of the bulk solution further highlighting the complex and variable nature of the solvent. 
To date, immense research effort has enabled construction of well-defined and extensively characterised, 3D architectures, many of which display unique properties in water. These research advances also supported the development of the first applications for watersoluble 3D assemblies, including their use in the development of contrast agents, as novel drug delivery vehicles, in catalysis and protein binding. Going forward it is proposed that collaborative, multi-disciplinary research programmes drawing on the complementary research expertise of physical, computational and supramolecular chemists, together with biotechnologists should enable a more detailed explanation for the origins of these unique properties. In turn, this will enable constructs with increasing complexity to be rationally assembled, thus expanding their application to, as yet, unperceived applications.

\section{$\underline{\text { Acknowledgements }}$}

We thank Jonathan Foster, Louise Natrajan, Jonathan Nitschke, Fangting Yu and Han Zuilhof for helpful discussions during the preparation of the manuscript. IAR thanks the University of Manchester for the Dame Kathleen Ollerenshaw Fellowship.

\section{$\underline{\text { References }}$}

[1] M. J. Webber, R. Langer, Chem. Soc. Rev. 2017, 46, 6600.

[2] C. J. Brown, F. D. Toste, R. G. Bergman, K. N. Raymond, Chem. Rev. 2015, 115, 3012.

[3] Q. Wang, Z. Li, D.-D. Tao, Q. Zhang, P. Zhang, D.-P. Guo, Y.-B. Jiang, Chem. Commun. 2016, 52, 12929.

[4] S. A. P. van Rossum, M. Tena-Solsona, J. H. van Esch, R. Eelkema, J. Boekhoven, Chem. Soc. Rev. 2017, 46, 5519.

[5] D. Bhatia, S. Surana, S. Chakraborty, S. P. Koushika, Y. Krishnan, Nat. Commun. 2011, 2, 339. 
[6] M. A. Shannon, P. W. Bohn, M. Elimelech, J. G. Georgiadis, B. J. Mariñas, A. M. Mayes, Nature 2008, 452, 301.

[7] V. Alphand, G. Carrea, R. Wohlgemuth, R. Furstoss, J. M. Woodley, Trends Biotechnol. 2003, 21, 318 .

[8] J. M. Woodley, Trends Biotechnol. 2008, 26, 321.

[9] A. Schmid, J. S. Dordick, B. Hauer, A. Kiener, M. Wubbolts, B. Witholt, Nature 2001, 409, 258.

[10] E. Brini, C. J. Fennell, M. Fernandez-Serra, B. Hribar-Lee, M. Lukšič, K. A. Dill, Chem. Rev. 2017, 117, 12385.

[11] H. Sato, K. Teramoto, Y. Masumoto, N. Tezuka, K. Sakai, S. Ueda, Y. Totsuka, T. Shinada, M. Nishiyama, C. Wang, T. Kuzuyama, M. Uchiyama, Sci. Rep. 2015, 5, 18471

[12] P. Ball, Proc. Natl. Acad. Sci. U. S. A. 2017, 114, 13327.

[13] P. S. Cremer, A. H. Flood, B. C. Gibb, D. L. Mobley, Nat. Chem. 2017, 10, 8.

[14] D. Ben-Amotz, Ann. Rev. Phys. Chem. 2016, 67, 617.

[15] O. Björneholm, M. H. Hansen, A. Hodgson, L.-M. Liu, D. T. Limmer, A. Michaelides, P. Pedevilla, J. Rossmeisl, H. Shen, G. Tocci, E. Tyrode, M.-M. Walz, J. Werner, H. Bluhm, Chem. Rev. 2016, 116, 7698.

[16] N. F. A. van der Vegt, K. Haldrup, S. Roke, J. Zheng, M. Lund, H. J. Bakker, Chem. Rev. 2016, 116, 7626.

[17] N. Agmon, H. J. Bakker, R. K. Campen, R. H. Henchman, P. Pohl, S. Roke, M. Thämer, A. Hassanali, Chem. Rev. 2016, 116, 7642.

[18] M.-C. Bellissent-Funel, A. Hassanali, M. Havenith, R. Henchman, P. Pohl, F. Sterpone, D. van der Spoel, Y. Xu, A. E. Garcia, Chem. Rev. 2016, 116, 7673.

[19] P. Ball, Chem. Rev. 2008, 108, 74. 
[20] J. Chen, N. C. Seeman, Nature 1991, 350, 631.

[21] C. M. Erben, R. P. Goodman, A. J. Turberfield, Angew. Chem. Int. Ed. 2006, 45, 7414.

[22] F. Yu, V. M. Cangelosi, M. L. Zastrow, M. Tegoni, J. S. Plegaria, A. G. Tebo, C. S. Mocny, L. Ruckthong, H. Qayyum, V. L. Pecoraro, Chem. Rev. 2014, 114, 3495.

[23] T. R. Cook, P. J. Stang, Chem. Rev. 2015, 115, 7001.

[24] D. L. Caulder, K. N. Raymond, Acc. Chem. Res. 1999, 32, 975.

[25] C. Lin, Y. Liu, H. Yan, Biochem. 2009, 48, 1663.

[26] R. Crawford, C. M. Erben, J. Periz, L. M. Hall, T. Brown, A. J. Turberfield, A. N. Kapanidis, Angew. Chem. Int. Ed. 2013, 52, 2284.

[27] S. Turega, W. Cullen, M. Whitehead, C. A. Hunter, M. D. Ward, J. Am. Chem. Soc. 2014, 136,8475 .

[28] Y.-T. Lai, E. Reading, G. L. Hura, K.-L. Tsai, A. Laganowsky, F. J. Asturias, J. A. Tainer, C. V. Robinson, T. O. Yeates, Nat. Chem. 2014, 6, 1065.

[29] D. Fujita, Y. Ueda, S. Sato, N. Mizuno, T. Kumasaka, M. Fujita, Nature 2016, 540, 563.

[30] E. G. Percástegui, J. Mosquera, J. R. Nitschke, Angew. Chem. Int. Ed. 2017, 56, 9136.

[31] E. Krieg, M. M. C. Bastings, P. Besenius, B. Rybtchinski, Chem. Rev. 2016, 116, 2414

[32] E. A. Appel, J. del Barrio, X. J. Loh, O. A. Scherman, Chem. Soc. Rev. 2012, 41, 6195.

[33] S. Kubik, Acc. Chem. Res. 2017, 50, 2870.

[34] J. H. Jordan, B. C. Gibb, Chem. Soc. Rev. 2015, 44, 547.

[35] A. M. Wen, N. F. Steinmetz, Chem. Soc. Rev. 2016, 45, 4074. 
[36] S. F. M. van Dongen, S. Cantekin, J. A. A. W. Elemans, A. E. Rowan, R. J. M. Nolte, Chem. Soc. Rev. 2014, 43, 99.

[37] L. Yang, X. Tan, Z. Wang, X. Zhang, Chem. Rev. 2015, 115, 7196.

[38] E. Yashima, N. Ousaka, D. Taura, K. Shimomura, T. Ikai, K. Maeda, Chem. Rev. 2016, $116,13752$.

[39] G. V. Oshovsky, D. N. Reinhoudt, W. Verboom, Angew. Chem. Int. Ed. 2007, 46, 2366.

[40] H. Vardhan, M. Yusubov, F. Verpoort, Coord. Chem. Rev. 2016, 306, Part 1, 171.

[41] M. Yoshizawa, J. K. Klosterman, M. Fujita, Angew. Chem. Int. Ed. 2009, 48, 3418.

[42] S. H. A. M. Leenders, R. Gramage-Doria, B. de Bruin, J. N. H. Reek, Chem. Soc. Rev. 2015, 44, 433 .

[43] A. J. McConnell, C. S. Wood, P. P. Neelakandan, J. R. Nitschke, Chem. Rev. 2015, 115,7729 .

[44] R. W. Saalfrank, A. Stark, K. Peters, H. G. von Schnering, Angew. Chem. Int. Ed. Engl. 1988, 27, 851.

[45] D. L. Caulder, R. E. Powers, T. N. Parac, K. N. Raymond, Angew. Chem. Int. Ed. 1998, 37, 1840.

[46] M. M. J. Smulders, I. A. Riddell, C. Browne, J. R. Nitschke, Chem. Soc. Rev. 2013, 42,1728 .

[47] M. Whitehead, S. Turega, A. Stephenson, C. A. Hunter, M. D. Ward, Chem. Sci. 2013, 4,2744 .

[48] M. Yamashina, Y. Sei, M. Akita, M. Yoshizawa, Nat. Commun. 2014, 5.

[49] K. Yazaki, Y. Sei, M. Akita, M. Yoshizawa, Chem. Eur. J. 2016, 22, 17557.

[50] M. Otte, P. F. Kuijpers, O. Troeppner, I. Ivanović-Burmazović, J. N. H. Reek, B. de Bruin, Chem. Eur. J. 2014, 20, 4880. 
[51] I. A. Riddell, M. M. J. Smulders, J. K. Clegg, J. R. Nitschke, Chem. Commun. 2011, $47,457$.

[52] K. Wu, K. Li, Y.-J. Hou, M. Pan, L.-Y. Zhang, L. Chen, C.-Y. Su, Nat. Commun. 2016, 7, 10487.

[53] L. Catti, Q. Zhang, K. Tiefenbacher, Chem. Eur. J. 2016, 22, 9060.

[54] M. Yoshizawa, M. Tamura, M. Fujita, Science 2006, 312, 251.

[55] A. Natarajan, L. S. Kaanumalle, S. Jockusch, C. L. D. Gibb, B. C. Gibb, N. J. Turro, V. Ramamurthy, J. Am. Chem. Soc. 2007, 129, 4132.

[56] J. W. Leeland, F. J. White, J. B. Love, J. Am. Chem. Soc. 2011, 133, 7320.

[57] P. Mal, B. Breiner, K. Rissanen, J. R. Nitschke, Science 2009, 324, 1697.

[58] M. D. Pluth, R. G. Bergman, K. N. Raymond, Acc. Chem. Res. 2009, 42, 1650.

[59] M. D. Pluth, R. G. Bergman, K. N. Raymond, J. Am. Chem. Soc. 2007, 129, 11459.

[60] C. J. Hastings, M. D. Pluth, R. G. Bergman, K. N. Raymond, J. Am. Chem. Soc. 2010, $132,6938$.

[61] W. M. Hart-Cooper, K. N. Clary, F. D. Toste, R. G. Bergman, K. N. Raymond, J. Am. Chem. Soc. 2012, 134, 17873.

[62] P. Das, A. Kumar, P. Howlader, P. S. Mukherjee, Chem. Eur. J. 2017, 23, 12565.

[63] S. Turega, M. Whitehead, B. R. Hall, M. F. Haddow, C. A. Hunter, M. D. Ward, Chem. Commun. 2012, 48.

[64] W. Cullen, M. C. Misuraca, C. A. Hunter, N. H. Williams, M. D. Ward, Nat. Chem. 2016, 8, 231.

[65] W. Cullen, A. J. Metherell, A. B. Wragg, C. G. P. Taylor, N. H. Williams, M. D. Ward, J. Am. Chem. Soc. 2018, 140, 2821.

[66] P. Howlader, P. Das, E. Zangrando, P. S. Mukherjee, J. Am. Chem. Soc. 2016, 138, 1668 
[67] B. Roy, A. Devaraj, R. Saha, S. Jharimune, K.-W. Chi, P. S. Mukherjee, Chem. Eur. J. 2017, 23, 15704.

[68] P. F. Kuijpers, M. Otte, M. Dürr, I. Ivanović-Burmazović, J. N. H. Reek, B. de Bruin, ACS Catal. 2016, 6, 3106.

[69] W. Meng, J. K. Clegg, J. D. Thoburn, J. R. Nitschke, J. Am. Chem. Soc. 2011, 133, 13652.

[70] J. L. Bolliger, A. M. Belenguer, J. R. Nitschke, Angew. Chem. Int. Ed. 2013, 52, 7958.

[71] C. Sgarlata, J. S. Mugridge, M. D. Pluth, V. Zito, G. Arena, K. N. Raymond, Chem. Eur. J. 2017, 23, 16813.

[72] J. Monod, J. Wyman, J.-P. Changeux, J. Mol. Biol. 1965, 12, 88.

[73] C. M. Hong, D. M. Kaphan, R. G. Bergman, K. N. Raymond, F. D. Toste, J. Am. Chem. Soc. 2017, 139, 8013.

[74] A. J. Metherell, W. Cullen, N. H. Williams, M. D. Ward, Chem. Eur. J. 2018, 24, 1554.

[75] W. Cullen, S. Turega, C. A. Hunter, M. D. Ward, Chem. Sci. 2015, 6, 2790.

[76] C. G. P. Taylor, W. Cullen, O. M. Collier, M. D. Ward, Chem. Eur. J. 2017, 23, 206.

[77] F. Biedermann, V. D. Uzunova, O. A. Scherman, W. M. Nau, A. De Simone, J. Am. Chem. Soc. 2012, 134, 15318.

[78] M. M. J. Smulders, S. Zarra, J. R. Nitschke, J. Am. Chem. Soc. 2013, 135, 7039.

[79] P. Mal, D. Schultz, K. Beyeh, K. Rissanen, J. R. Nitschke, Angew. Chem. Int. Ed. 2008, 47, 8297.

[80] M. M. J. Smulders, J. R. Nitschke, Chem. Sci. 2012, 3, 785.

[81] S. Chen, K. Li, F. Zhao, L. Zhang, M. Pan, Y.-Z. Fan, J. Guo, J. Shi, C.-Y. Su, Nat. Commun. 2016, 7, 13169.

[82] W. Cullen, S. Turega, C. A. Hunter, M. D. Ward, Chem. Sci. 2015, 6, 625. 
[83] B. Therrien, CrystEngComm 2015, 17, 484.

[84] B. Therrien, Chem. Eur. J. 2013, 19, 8378.

[85] B. Therrien, G. Süss-Fink, P. Govindaswamy, A. K. Renfrew, P. J. Dyson, Angew. Chem. Int. Ed. 2008, 47, 3773.

[86] J. W. Yi, N. P. E. Barry, M. A. Furrer, O. Zava, P. J. Dyson, B. Therrien, B. H. Kim, Bioconjug. Chem. 2012, 23, 461.

[87] F. Schmitt, J. Freudenreich, N. P. E. Barry, L. Juillerat-Jeanneret, G. Süss-Fink, B. Therrien, J. Am. Chem. Soc. 2012, 134, 754.

[88] S. K. Samanta, D. Moncelet, V. Briken, L. Isaacs, J. Am. Chem. Soc. 2016, 138, 14488.

[89] Y.-R. Zheng, K. Suntharalingam, T. C. Johnstone, S. J. Lippard, Chem. Sci. 2015, 6, 1189.

[90] F. Ibukuro, T. Kusukawa, M. Fujita, J. Am. Chem. Soc. 1998, 120, 8561.

[91] W. Cullen, C. A. Hunter, M. D. Ward, Inorg. Chem. 2015, 54, 2626.

[92] M. Scherer, D. L. Caulder, D. W. Johnson, K. N. Raymond, Angew. Chem. Int. Ed. 1999, 38, 1587.

[93] S. Wang, T. Sawada, K. Ohara, K. Yamaguchi, M. Fujita, Angew. Chem. Int. Ed. 2016, 55, 2063.

[94] S. Wang, T. Sawada, M. Fujita, Chem. Commun. 2016, 52, 11653.

[95] D. Zhang, T. K. Ronson, J. Mosquera, A. Martinez, L. Guy, J. R. Nitschke, J. Am. Chem. Soc. 2017, 139, 6574.

[96] S. M. McNeill, D. Preston, J. E. M. Lewis, A. Robert, K. Knerr-Rupp, D. O. Graham, J. R. Wright, G. I. Giles, J. D. Crowley, Dalton Trans. 2015, 44, 11129. 
[97] A. Adamski, M. A. Fik, M. Kubicki, Z. Hnatejko, D. Gurda, A. FedorukWyszomirska, E. Wyszko, D. Kruszka, Z. Dutkiewicz, V. Patroniak, New J. Chem. 2016, 40, 7943.

[98] U. Golla, A. Adhikary, A. K. Mondal, R. S. Tomar, S. Konar, Dalton Trans. 2016, 45, 11849.

[99] M. J. Hannon, V. Moreno, M. J. Prieto, E. Moldrheim, E. Sletten, I. Meistermann, C. J. Isaac, K. J. Sanders, A. Rodger, Angew. Chem. Int. Ed. 2001, 40, 879.

[100] A. C. G. Hotze, B. M. Kariuki, M. J. Hannon, Angew. Chem. Int. Ed. 2006, 45, 4839.

[101] S. E. Howson, A. Bolhuis, V. Brabec, G. J. Clarkson, J. Malina, A. Rodger, P. Scott, Nature Chem. 2011, 4, 31.

[102] A. Oleksi, A. G. Blanco, R. Boer, I. Usón, J. Aymamí, A. Rodger, M. J. Hannon, M. Coll, Angew. Chem. Int. Ed. 2006, 45, 1227.

[103] L. Cardo, V. Sadovnikova, S. Phongtongpasuk, N. J. Hodges, M. J. Hannon, Chem. Commun. 2011, 47, 6575.

[104] C. Zhao, J. Geng, L. Feng, J. Ren, X. Qu, Chem. Eur. J. 2011, 17, 8209.

[105] J. Malina, P. Scott, V. Brabec, Dalton Trans. 2015, 44, 14656.

[106] J. Malina, J. Hannon Michael, V. Brabec, FEBS J. 2013, 281, 987.

[107] J. Malina, J. Hannon Michael, V. Brabec, Chem. Eur. J. 2015, 21, 11189.

[108] R. A. Kaner, S. J. Allison, A. D. Faulkner, R. M. Phillips, D. I. Roper, S. L. Shepherd, D. H. Simpson, N. R. Waterfield, P. Scott, Chem. Sci. 2016, 7, 951.

[109] M. Li, S. E. Howson, K. Dong, N. Gao, J. Ren, P. Scott, X. Qu, J. Am. Chem. Soc. 2014, 136, 11655.

[110] H. Yu, M. Li, G. Liu, J. Geng, J. Wang, J. Ren, C. Zhao, X. Qu, Chem. Sci. 2012, 3, 3145 .

[111] N. C. Seeman, J. Theor. Biol. 1982, 99, 237. 
[112] Y. Zhang, N. C. Seeman, J. Am. Chem. Soc. 1994, 116, 1661.

[113] F. A. Aldaye, H. F. Sleiman, Angew. Chem. Int. Ed. 2006, 45, 2204.

[114] Y. Ma, Z. Wang, Y. Ma, Z. Han, M. Zhang, H. Chen, Y. Gu, Angew. Chem. Int. Ed. 2018, 57, 5389.

[115] R. P. Goodman, I. A. T. Schaap, C. F. Tardin, C. M. Erben, R. M. Berry, C. F. Schmidt, A. J. Turberfield, Science 2005, 310, 1661.

[116] R. P. Goodman, M. Heilemann, S. Doose, C. M. Erben, A. N. Kapanidis, A. J. Turberfield, Nat. Nanotechnol. 2008, 3, 93.

[117] S. M. Douglas, I. Bachelet, G. M. Church, Science 2012, 335, 831.

[118] D. Mitra, N. Di Cesare, H. F. Sleiman, Angew. Chem. Int. Ed. 2004, 43, 5804.

[119] J. S. Choi, C. W. Kang, K. Jung, J. W. Yang, Y.-G. Kim, H. Han, J. Am. Chem. Soc. 2004, 126, 8606 .

[120] F. A. Aldaye, H. F. Sleiman, J. Am. Chem. Soc. 2007, 129, 13376.

[121] M. Scheffler, A. Dorenbeck, S. Jordan, M. Wüstefeld, G. von Kiedrowski, Angew. Chem. Int. Ed. 1999, 38, 3311.

[122] J. Zimmermann, M. P. J. Cebulla, S. Mönninghoff, G. von Kiedrowski, Angew. Chem. Int. Ed. 2008, 47, 3626.

[123] H. Yang, C. K. McLaughlin, F. A. Aldaye, G. D. Hamblin, A. Z. Rys, I. Rouiller, H. F. Sleiman, Nat. Chem. 2009, 1, 390.

[124] J. Nangreave, D. Han, Y. Liu, H. Yan, Curr. Opin. Chem. Biol. 2010, 14, 608.

[125] F. Hong, F. Zhang, Y. Liu, H. Yan, Chem. Rev. 2017, 117, 12584.

[126] Y. Ke, J. Sharma, M. Liu, K. Jahn, Y. Liu, H. Yan, Nano Lett. 2009, 9, 2445.

[127] D. Han, S. Pal, J. Nangreave, Z. Deng, Y. Liu, H. Yan, Science 2011, 332, 342.

[128] W. M. Shih, J. D. Quispe, G. F. Joyce, Nature 2004, 427, 618.

[129] Y. He, C. Mao, Chem. Commun. 2006, 0, 968. 
[130] Y. He, T. Ye, M. Su, C. Zhang, A. E. Ribbe, W. Jiang, C. Mao, Nature 2008, 452, 198.

[131] Y. He, Y. Tian, Y. Chen, Z. Deng, A. E. Ribbe, C. Mao, Angew. Chem. Int. Ed. 2005, $44,6694$.

[132] C. Zhang, M. Su, Y. He, X. Zhao, P.-a. Fang, A. E. Ribbe, W. Jiang, C. Mao, Proc. Natl Acad. Sci. U. S. A. 2008, 105, 10665.

[133] Y. He, Y. Tian, A. E. Ribbe, C. Mao, J. Am. Chem. Soc. 2006, 128, 15978.

[134] C. Zhang, S. H. Ko, M. Su, Y. Leng, A. E. Ribbe, W. Jiang, C. Mao, J. Am. Chem. Soc. 2009, 131, 1413.

[135] T. G. W. Edwardson, K. M. M. Carneiro, C. K. McLaughlin, C. J. Serpell, H. F. Sleiman, Nat. Chem. 2013, 5, 868.

[136] P. Chidchob, T. G. W. Edwardson, C. J. Serpell, H. F. Sleiman, J. Am. Chem. Soc. 2016, 138,4416 .

[137] F. A. Aldaye, H. F. Sleiman, J. Am. Chem. Soc. 2007, 129, 4130.

[138] Y. Tian, T. Wang, W. Liu, H. L. Xin, H. Li, Y. Ke, W. M. Shih, O. Gang, Nat. Nanotechnol. 2015, 10, 637.

[139] D. Bhatia, S. Mehtab, R. Krishnan, S. S. Indi, A. Basu, Y. Krishnan, Angew. Chem. Int. Ed. 2009, 48, 4134.

[140] M. Sandro, J. J. Rebek, Chem. Eur. J. 1998, 4, 1016.

[141] H. Lee, A. K. R. Lytton-Jean, Y. Chen, K. T. Love, A. I. Park, E. D. Karagiannis, A. Sehgal, W. Querbes, C. S. Zurenko, M. Jayaraman, C. G. Peng, K. Charisse, A. Borodovsky, M. Manoharan, J. S. Donahoe, J. Truelove, M. Nahrendorf, R. Langer, D. G. Anderson, Nat. Nanotechnol. 2012, 7, 389.

[142] A. S. Walsh, H. Yin, C. M. Erben, M. J. A. Wood, A. J. Turberfield, ACS Nano 2011, 5,5427 . 
[143] G. Vindigni, S. Raniolo, A. Ottaviani, M. Falconi, O. Franch, B. R. Knudsen, A. Desideri, S. Biocca, ACS Nano 2016, 10, 5971.

[144] A. Banerjee, D. Bhatia, A. Saminathan, S. Chakraborty, S. Kar, Y. Krishnan, Angew. Chem. Int. Ed. 2013, 52, 6854.

[145] J. Li, H. Pei, B. Zhu, L. Liang, M. Wei, Y. He, N. Chen, D. Li, Q. Huang, C. Fan, ACS Nano 2011, 5, 8783.

[146] K. E. Bujold, J. C. C. Hsu, H. F. Sleiman, J. Am. Chem. Soc. 2016, 138, 14030.

[147] X. Liu, Y. Xu, T. Yu, C. Clifford, Y. Liu, H. Yan, Y. Chang, Nano Lett. 2012, 12, 4254.

[148] S. Juul, F. Iacovelli, M. Falconi, S. L. Kragh, B. Christensen, R. Frøhlich, O. Franch, E. L. Kristoffersen, M. Stougaard, K. W. Leong, Y.-P. Ho, E. S. Sørensen, V. Birkedal, A. Desideri, B. R. Knudsen, ACS Nano 2013, 7, 9724.

[149] J. D. Flory, S. Shinde, S. Lin, Y. Liu, H. Yan, G. Ghirlanda, P. Fromme, J. Am. Chem. Soc. 2013, 135, 6985.

[150] T. Nugent, D. T. Jones, Proc. Natl Acad. Sci. U. S. A. 2012, 109, E1540.

[151] C. W. Wood, M. Bruning, A. Á. Ibarra, G. J. Bartlett, A. R. Thomson, R. B. Sessions, R. L. Brady, D. N. Woolfson, Bioinformatics 2014, 30, 3029.

[152] K. W. Kaufmann, G. H. Lemmon, S. L. DeLuca, J. H. Sheehan, J. Meiler, Biochem. 2010, 49, 2987.

[153] Z. Dauter, IUCrJ 2015, $2,164$.

[154] P.-S. Huang, S. E. Boyken, D. Baker, Nature 2016, 537, 320.

[155] M. Mason Jody, M. Arndt Katja, ChemBioChem 2004, 5, 170.

[156] M. G. Oakley, J. J. Hollenbeck, Curr. Opin. Struct. Biol. 2001, 11, 450.

[157] F. Thomas, A. Niitsu, A. Oregioni, G. J. Bartlett, D. N. Woolfson, Biochem. 2017, 56, 6544. 
[158] B. Berger, D. B. Wilson, E. Wolf, T. Tonchev, M. Milla, P. S. Kim, Proc. Natl Acad. Sci. U. S. A. 1995, 92, 8259.

[159] W. D. Kohn, C. M. Kay, R. S. Hodges, J. Mol. Biol. 1998, 283, 993.

[160] C. Aronsson, S. Dånmark, F. Zhou, P. Öberg, K. Enander, H. Su, D. Aili, Sci. Rep. 2015, 5, 14063.

[161] K. Suzuki, H. Hiroaki, D. Kohda, H. Nakamura, T. Tanaka, J. Am. Chem. Soc. 1998, 120,13008 .

[162] M. L. Zastrow, A. F. A. Peacock, J. A. Stuckey, V. L. Pecoraro, Nat. Chem. 2011, 4, 118.

[163] M. R. Berwick, D. J. Lewis, A. W. Jones, R. A. Parslow, T. R. Dafforn, H. J. Cooper, J. Wilkie, Z. Pikramenou, M. M. Britton, A. F. A. Peacock, J. Am. Chem. Soc. 2014, 136,1166

[164] X. Li, K. Suzuki, K. Kanaori, K. Tajima, A. Kashiwada, H. Hiroaki, D. Kohda, T. Tanaka, Protein Sci. 2000, 9, 1327.

[165] L. Regan, N. D. Clarke, Biochem. 1990, 29, 10878.

[166] L. Ruckthong, M. L. Zastrow, J. A. Stuckey, V. L. Pecoraro, J. Am. Chem. Soc. 2016, $138,11979$.

[167] C. M. Hong, M. Morimoto, E. A. Kapustin, N. Alzakhem, R. G. Bergman, K. N. Raymond, F. D. Toste, J. Am. Chem. Soc. 2018, 140, 6591.

[168] I. A. Riddell, Y. R. Hristova, J. K. Clegg, C. S. Wood, B. Breiner, J. R. Nitschke, J. Am. Chem. Soc. 2013, 135, 2723.

[169] W. D. Kohn, C. M. Kay, B. D. Sykes, R. S. Hodges, J. Am. Chem. Soc. 1998, 120, 1124.

[170] A. R. Stefankiewicz, M. R. Sambrook, J. K. M. Sanders, Chem. Sci 2012, 3, 2326. 
[171] N. Ponnuswamy, F. B. L. Cougnon, J. M. Clough, G. D. Pantoş, J. K. M. Sanders, Science 2012, 338, 783.

[172] F. B. L. Cougnon, J. K. M. Sanders, Acc. Chem. Res. 2012, 45, 2211.

[173] A. R. Stefankiewicz, M. R. Sambrook, J. K. M. Sanders, Chem. Sci. 2012, 3, 2326.

[174] W. M. Park, M. Bedewy, K. K. Berggren, A. E. Keating, Sci. Rep. 2017, 7, 10577.

[175] R. J. Radford, F. A. Tezcan, J. Am. Chem. Soc. 2009, 131, 9136.

[176] A. L. Boyle, E. H. C. Bromley, G. J. Bartlett, R. B. Sessions, T. H. Sharp, C. L. Williams, P. M. G. Curmi, N. R. Forde, H. Linke, D. N. Woolfson, J. Am. Chem. Soc. 2012, 134,15457

[177] Y. Bai, Q. Luo, W. Zhang, L. Miao, J. Xu, H. Li, J. Liu, J. Am. Chem. Soc. 2013, 135, 10966.

[178] R. Wang, S. Qiao, L. Zhao, C. Hou, X. Li, Y. Liu, Q. Luo, J. Xu, H. Li, J. Liu, Chem. Commun. 2017, 53, 10532.

[179] J. M. Fletcher, R. L. Harniman, F. R. H. Barnes, A. L. Boyle, A. Collins, J. Mantell, T. H. Sharp, M. Antognozzi, P. J. Booth, N. Linden, M. J. Miles, R. B. Sessions, P. Verkade, D. N. Woolfson, Science 2013, 340, 595.

[180] J. R. Cochrane, A. Schmitt, U. Wille, C. A. Hutton, Chem. Commun. 2013, 49, 5504.

[181] E. Bartolami, J. Knoops, Y. Bessin, M. Fossépré, J. Chamieh, P. Dumy, M. Surin, S. Ulrich, Chem. Eur. J. 2017, 23, 14323.

[182] H. Gradišar, S. Božič, T. Doles, D. Vengust, I. Hafner-Bratkovič, A. Mertelj, B. Webb, A. Šali, S. Klavžar, R. Jerala, Nat. Chem. Biol. 2013, 9, 362.

[183] N. Kobayashi, K. Yanase, T. Sato, S. Unzai, M. H. Hecht, R. Arai, J. Am. Chem. Soc. 2015, 137, 11285 .

[184] S. Badieyan, A. Sciore, J. D. Eschweiler, P. Koldewey, A. S. Cristie-David, B. T. Ruotolo, J. C. A. Bardwell, M. Su, E. N. G. Marsh, ChemBioChem 2017, 18, 1888. 
[185] Y.-T. Lai, D. Cascio, T. O. Yeates, Science 2012, 336, 1129.

[186] P. Caravan, J. M. Greenwood, J. T. Welch, S. J. Franklin, Chem. Commun. 2003, 0, 2574.

[187] M. R. Berwick, L. N. Slope, C. F. Smith, S. M. King, S. L. Newton, R. B. Gillis, G. G. Adams, A. J. Rowe, S. E. Harding, M. M. Britton, A. F. A. Peacock, Chem. Sci. 2016, 7, 2207.

[188] M. Tegoni, F. Yu, M. Bersellini, J. E. Penner-Hahn, V. L. Pecoraro, Proc. Natl Acad. Sci. U. S. A. 2012, 109, 21234.

[189] V. M. Cangelosi, A. Deb, J. E. Penner-Hahn, V. L. Pecoraro, Angew. Chem. Int. Ed. 2014, 53, 7900 .

[190] S. Chakraborty, J. Y. Kravitz, P. W. Thulstrup, L. Hemmingsen, W. F. DeGrado, V. L. Pecoraro, Angew. Chem. Int. Ed. 2011, 50, 2049.

[191] Z. Gasyna, A. Zelazowski, A. R. Green, E. Ough, M. J. Stillman, Inorg. Chim. Acta 1988, $153,115$.

[192] A. R. Green, M. J. Stillman, Inorg. Chim. Acta 1994, 226, 275.

[193] J. Hong, O. A. Kharenko, J. Fan, F. Xie, A. K. Petros, B. R. Gibney, M. Y. Ogawa, Angew. Chem. Int. Ed. 2006, 45, 6137.

[194] G. W. Collie, R. Bailly, K. Pulka-Ziach, C. M. Lombardo, L. Mauran, N. TaibMaamar, J. Dessolin, C. D. Mackereth, G. Guichard, J. Am. Chem. Soc. 2017, 139, 6128.

[195] J.-m. Suk, K.-S. Jeong, J. Am. Chem. Soc. 2008, 130, 11868.

[196] S. L. Schreiber, Nature Chem. Biol. 2005, 1, 64.

[197] G. M. T. Cooper, The Cell: A Molecular Approach. 2nd edition. Sunderland (MA): Sinauer Associates; 2000. Signaling Molecules and Their Receptors. Available from: https://www.ncbi.nlm.nih.gov/books/NBK9924/. 
[198] J. Hahn, S. F. J. Wickham, W. M. Shih, S. D. Perrault, ACS Nano 2014, 8, 8765.

[199] T. Kikuchi, S. Sato, D. Fujita, M. Fujita, Chem. Sci. 2014, 5, 3257.

[200] C. K. McLaughlin, G. D. Hamblin, H. F. Sleiman, Chem. Soc. Rev. 2011, 40, 5647.

[201] P. Paolo, M. Tiziana, R. Nino, D. R. Salahub, ChemCatChem 2017, 9, 1047. 


\section{Bibliographic sketches}

Lauren Taylor completed her MChem under the supervision of Prof. Patrick McGowan at The University of Leeds where she synthesised ruthenium arene complexes as potential anticancer drugs. In 2017 she began her PhD with Dr. Imogen Riddell at The University of Manchester, where her current research is focussed on the synthesis of supramolecular architectures for protein encapsulation and stabilisation.

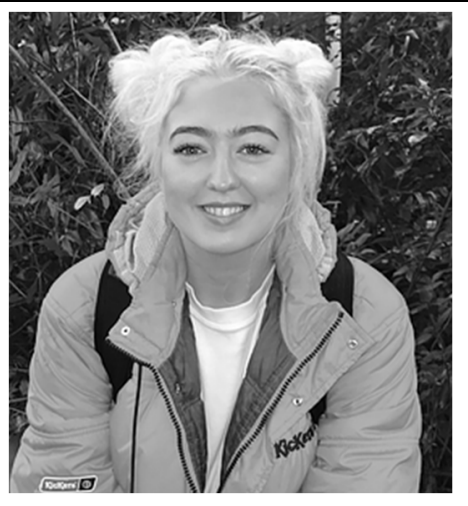

Imogen Riddell completed her $\mathrm{PhD}$ with Jonathan Nitschke (University of Cambridge) exploring the design and applications of new supramolecular cages. She then went on to a postdoctoral appointment with Stephen Lippard (Massachusetts Institute of Technology) where she investigated the mechanism of action of non-classical anticancer agents. In 2017 she was appointed as a Dame Kathleen Ollerenshaw Fellow at the University of Manchester where her research focuses on utilising self-assembled

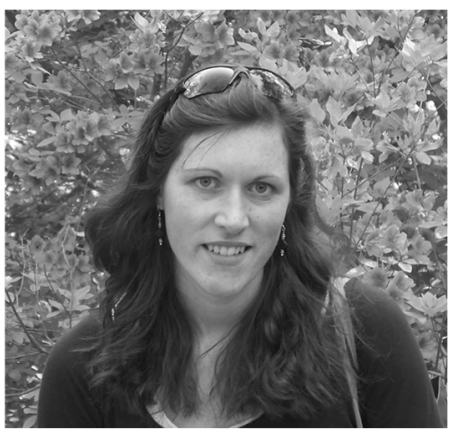
constructs for applications including protein stabilisation.

Maarten Smulders obtained his $\mathrm{PhD}$ in supramolecular chemistry with Bert Meijer (Eindhoven University of Technology). After postdoctoral stays with Jonathan Nitschke (Cambridge University) and Jeroen Cornelissen (Twente University), he joined Wageningen University in 2013 as an assistant professor, starting his independent research, funded by a NWO Veni grant. His current research interests are in supramolecular chemistry and dynamic-covalent (polymer) chemistry. In 2018 he was awarded a NWO Vidi grant, to fortify his research program in the latter area.

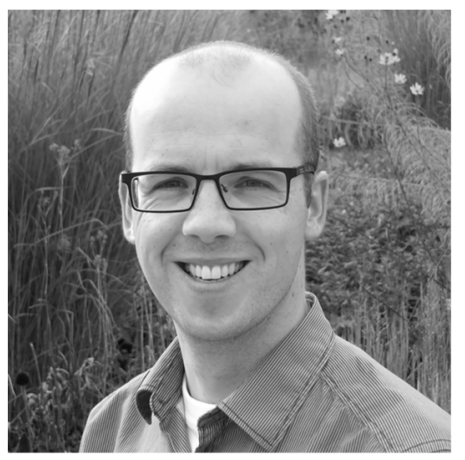

